\title{
Photooxidase Mimicking with Adaptive Coordination Molecular Capsules
}

Dan-Ni Yan, ${ }^{\dagger}, \S$ Li-Xuan Cai, ${ }^{\dagger}$ Pei-Ming Cheng, ${ }^{\dagger}$ Shao-Jun Hu, ${ }^{\dagger}, \S$ Li-Peng Zhou,,${ }^{\dagger}$ Qing-Fu Sun $* \dagger, \S$

†State Key Laboratory of Structural Chemistry, Fujian Institute of Research on the Structure of Matter, Chinese Academy of Sciences, Fuzhou 350002, PR China

§University of Chinese Academy of Sciences, Beijing 100049, PR China

*Corresponding Author. Email: qfsun@fjirsm.ac.cn.

\section{Table of Content}

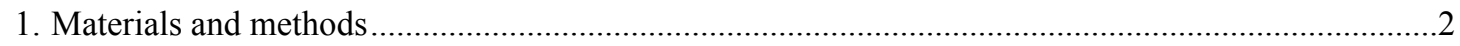

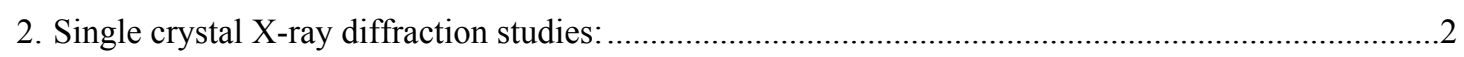

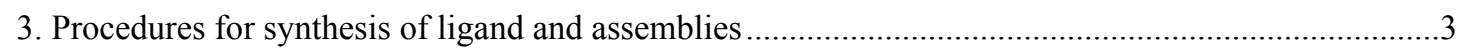

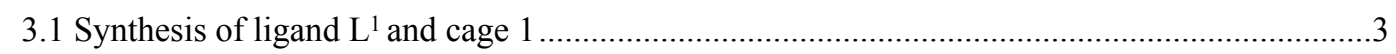

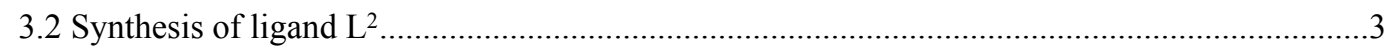

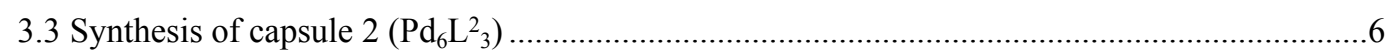

4. The host-guest behavior and structure conversion of capsule 2 .................................................. 9

4.1 General procedure for the preparation of host-guest complexes:.........................................

4.2 Formation of inclusion complex (adamantane derivatives) $)_{8} \subset$ capsule $2:$...............................

4.3 Formation of inclusion complex (methyl(4-nitrophenyl)sulfane) $)_{3} \subset$ bowl 3:........................14

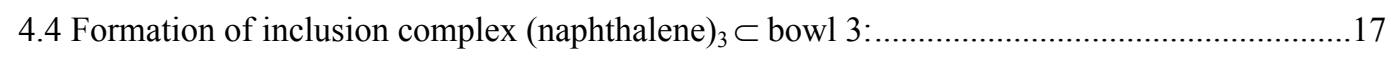

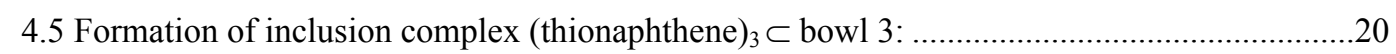

4.6 In-situ guest-template self-assembly of $\mathrm{L}^{2}, \mathrm{Pd}$, and naphthalene...........................................23

4.7 The conversion from capsule 2 to bowl 3 over time: .............................................................23

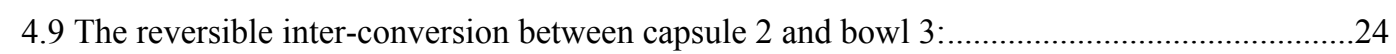

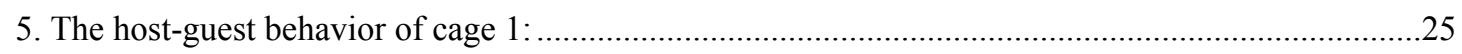

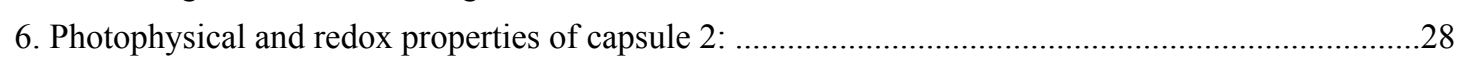

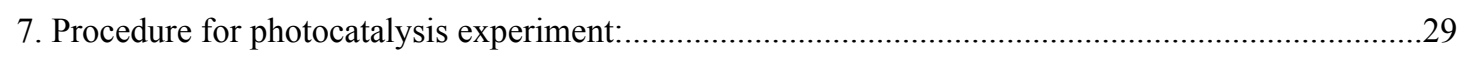

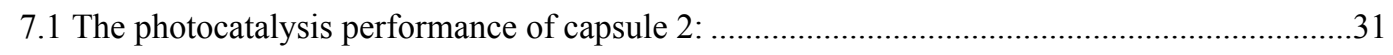

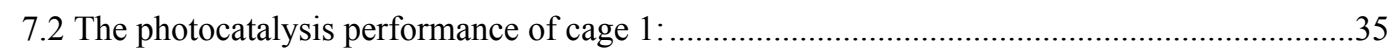

7.3 Control experiments and proposed mechanism: ....................................................................

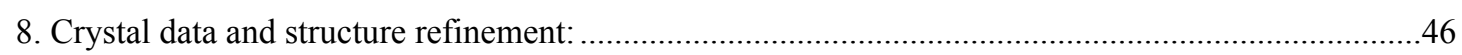

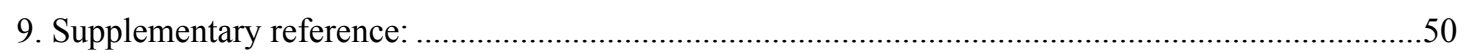




\section{Materials and methods}

Unless otherwise stated, all chemicals and solvents were purchased from commercial companies and used without further purification. 2, 4, 6-Tris(4-pyridyl)-1,3,5-triazine (TPT) and 9,10-bis(bromomethyl)anthracene were synthesized as reported previously in the literature. ${ }^{S 1, S 2}$ Deuterated solvents were purchased from Admas, Sigma-Aldrich and J\&K scientific. 1D and 2D NMR spectra were measured on a Bruker Biospin Avance III (400 MHz) and a JEOL ECZ600S $(600 \mathrm{MHz})$ spectrometer. The NMR chemical shifts $(\delta)$ are reported in ppm (parts per million) with respect to solvent signal originated due to incomplete deuteration ( $\delta=$ 4.79 for $\mathrm{D}_{2} \mathrm{O}, \delta=2.50$ for DMSO- $d_{6}, \delta=7.26$ for $\mathrm{CDCl}_{3}$ in ${ }^{1} \mathrm{H} \mathrm{NMR}$ ). UV-Vis absorption spectra were recorded on UV-2700 UV-Visible spectrophotometer from SHIMADZU Corporation. ESI-TOF-MS spectra were recorded on an Impact II UHR-TOF mass spectrometry from Bruker or LC-QTOF-MS (G6520B). Data analyses were conducted with the Bruker Data Analysis software (Version 4.3) and simulations were performed with the Bruker Isotope Pattern software or thermo Xcalibur Qual Browser software (Thermo Foundation 2.0). Electron chemical analysis was performed on CHI 604E analyzer. All Cyclic voltammetry (CV) experiments were carried out in argon-purged solutions of DMSO with $0.1 \mathrm{M}$ of tetra(n-butyl)ammonium hexa-fluorophosphate $\left(\mathrm{TBAPF}_{6}\right)$ as electrolyte at a scan rate of $100 \mathrm{mV} / \mathrm{s}$ at rt. A glassy carbon was used as the working electrode, $\mathrm{Ag} / \mathrm{AgCl}$ as the reference electrode, and Pt wire as the counter electrode. The blue LED light sources used for all the photoreactions in this research were from Wuhan Geao Instruments Science \& Technology Co., Ltd (China) $(\lambda>450 \mathrm{~nm} ; 1 \mathrm{~W}$ for every lamp beads; every Schlenk tube was irradiated by 6 lamp beads from the side).

\section{Single crystal X-ray diffraction studies:}

X-ray diffraction studies for ligand $\mathrm{L}^{2}, \mathbf{A D C} \subset$ capsule $\mathbf{2}, \mathbf{M N S} \mathbf{S}_{3} \subset$ bowl $\mathbf{3}$ were carried out at the BL17B macromolecular crystallography beamline in SSRF. The collected diffraction data were processed with the APEX-III software or the HKL3000 software program. The structures were solved by direct method and refined by full-matrix least-squares on $F^{2}$ with anisotropic displacement using the SHELX software package. ${ }^{S 3}$ In the case of host-guest complexes, solvent molecules were highly disordered and could not be reasonably located. These residual intensities were removed by PLATON/SQUEEZE routine. ${ }^{S 4}$

Crystal data for ligand $\mathrm{L}^{2}$ : Space group $P-1, \mathrm{a}=7.917(3) \AA, \mathrm{b}=10.411(5) \AA, \mathrm{c}=$ 14.793(7) $\AA, \mathrm{V}=1108.3(9) \AA^{3}, \mathrm{Z}=1, \mathrm{~T}=100 \mathrm{~K}$. Anisotropic least-squares refinement on 2094 independent merged reflections $\left(\mathrm{R}_{\text {int }}=0.0482\right)$ converged at residual $w R_{2}=0.3962$ for all data; residual $R_{1}=0.1236$ for 3970 observed data $[I>$ $2 \sigma(I)]$, and goodness of fit $(\mathrm{GOF})=1.133$. (CCDC number: 2084602)

Crystal data for $\mathbf{A D C} \mathbf{C}_{8} \subset$ capsule 2: Space group $P 2_{1} / c, \mathrm{a}=22.611(10) \AA, \mathrm{b}=$ 30.064(14) $\AA, c=55.373(3) \AA, V=37506(3) \AA^{3}, Z=4, T=100 \mathrm{~K}$. Anisotropic least-squares refinement for the framework atoms and isotropic refinement for the other atoms on 29497 independent merged reflections $\left(\mathrm{R}_{\text {int }}=0.0784\right)$ converged at 
residual $w R_{2}=0.3797$ for all data; residual $R_{1}=0.1284$ for 107402 observed data $[I>$ $2 \sigma(I)]$, and goodness of fit $(\mathrm{GOF})=1.606$. (CCDC number: 2084605$)$

Crystal data for $\mathbf{M N S}_{3} \subset$ bowl 3: Space group $P-1, \mathrm{a}=20.535(4) \AA, \mathrm{b}=$ 21.230(4) $\AA, \mathrm{c}=29.674(6) \AA, \mathrm{V}=11425(5) \AA^{3}, \mathrm{Z}=2, \mathrm{~T}=100 \mathrm{~K}$. Anisotropic least-squares refinement for the framework atoms and isotropic refinement for the other atoms on 26300 independent merged reflections $\left(\mathrm{R}_{\text {int }}=0.0756\right)$ converged at residual $w R_{2}=0.4271$ for all data; residual $R_{1}=0.1519$ for 48890 observed data $[I>$ $2 \sigma(I)]$, and goodness of fit $(\mathrm{GOF})=1.474$. (CCDC number: 2084606)

\section{Procedures for synthesis of ligand and assemblies}

\subsection{Synthesis of ligand $L^{1}$ and cage 1}

Ligand $\mathrm{L}^{1}$ and cage 1 were synthesized according to previous work. ${ }^{S 5}$

\subsection{Synthesis of ligand $L^{2}$}

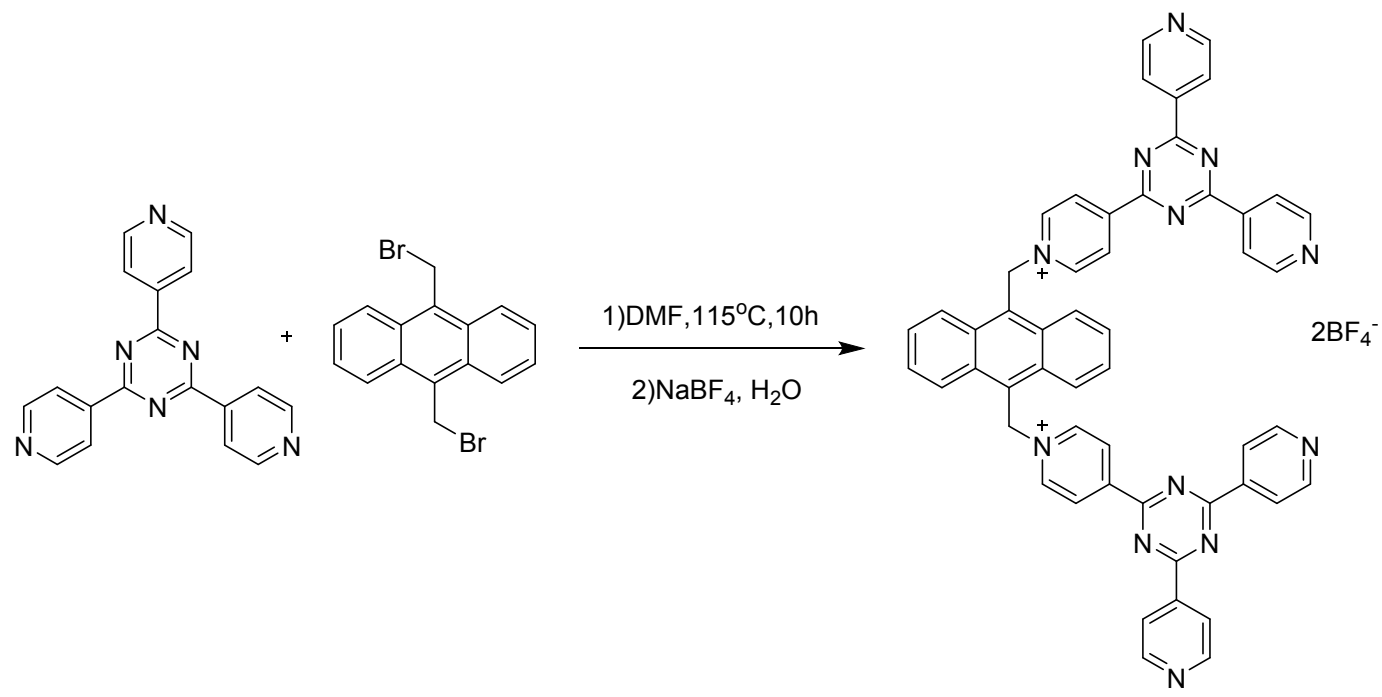

Scheme S1. Synthesis of ligand $\mathrm{L}^{2} \cdot\left(\mathrm{BF}_{4}\right)_{2}$.

2, 4, 6-Tris(4-pyridyl)-1,3,5-triazine (312 $\mathrm{mg}, 1.00 \mathrm{mmol}$ ) was added to dry dimethylformamide $(100 \mathrm{~mL})$ under an atmosphere of $\mathrm{N}_{2}$ and the suspension was stirred at $115^{\circ} \mathrm{C}$ until it became clear. The solution was treated drop-wise with 9,10 -bis(bromomethyl)anthracene (104 $\mathrm{mg}, 0.286 \mathrm{mmol}$ ) dissolved in a small amount of $\mathrm{DMF}$ and then maintained at $115^{\circ} \mathrm{C}$ for $10 \mathrm{~h}$, affording a light-orange precipitate. The precipitate was filtered and washed with dimethylformamide and ethanol, then dry in a vacuum oven. The filter cake was dissolved in hot water to remove excess TPT, followed by the addition of an excess of sodium tetrafluoroborate $\left(\mathrm{NaBF}_{4}\right)$, giving an orange solid of pure $\mathrm{L}^{2} \cdot\left(\mathrm{BF}_{4}\right)_{2}(\mathrm{M}=1002.53 \mathrm{~g} / \mathrm{mol}, 199 \mathrm{mg}$, yield $69.5 \%)$ that was collected by filtration. ${ }^{1} \mathrm{H}$ NMR $\left(600 \mathrm{MHz}, \mathrm{DMSO}-d_{6}, 298 \mathrm{~K}\right): \delta 9.28(\mathrm{~d}, J=7.2 \mathrm{~Hz}$, $4 \mathrm{H}), 9.20(\mathrm{~d}, J=7.2 \mathrm{~Hz}, 4 \mathrm{H}), 8.98(\mathrm{~d}, J=6.0 \mathrm{~Hz}, 8 \mathrm{H}), 8.71-8.67(\mathrm{~m}, 4 \mathrm{H}), 8.67(\mathrm{~d}, J=$ $6.0 \mathrm{~Hz}, 8 \mathrm{H}), 7.84(\mathrm{dd}, J=7.2,3.6 \mathrm{~Hz}, 4 \mathrm{H}), 7.28(\mathrm{~s}, 4 \mathrm{H}) .{ }^{13} \mathrm{C}$ NMR $(101 \mathrm{MHz}$, DMSO-d $d_{6}$ ): $\delta 171.10(\mathrm{~s}), 168.63$ (s), 150.28 (s), 149.74 (s), 146.02 (s), 144.06 (s), 


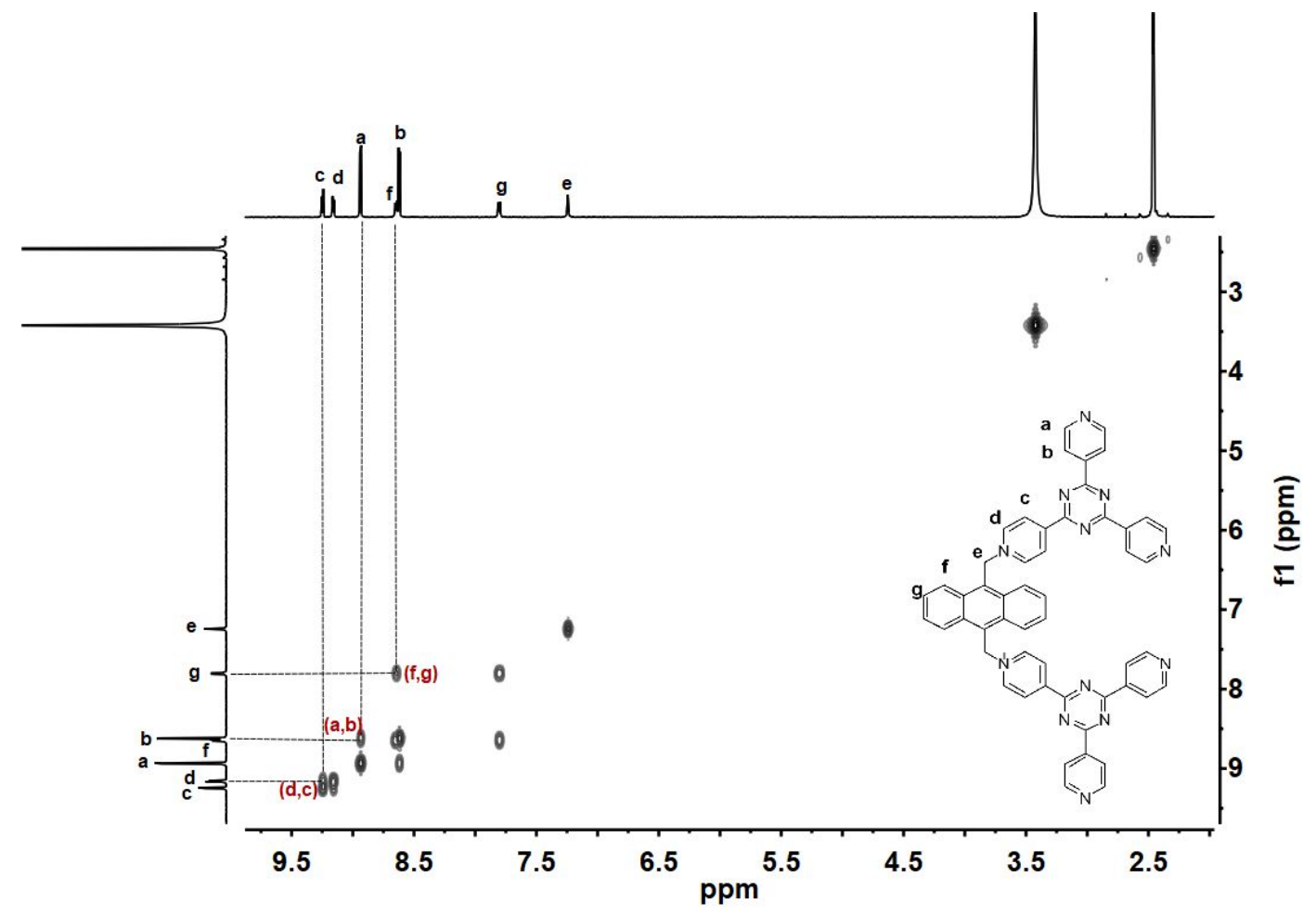

Figure S3. ${ }^{1} \mathrm{H}-{ }^{1} \mathrm{H}$ COSY NMR spectrum $\left(600 \mathrm{MHz}\right.$, DMSO- $\left.d_{6}, 298 \mathrm{~K}\right)$ of ligand $\mathrm{L}^{2} \cdot\left(\mathrm{BF}_{4}\right)_{2}$.

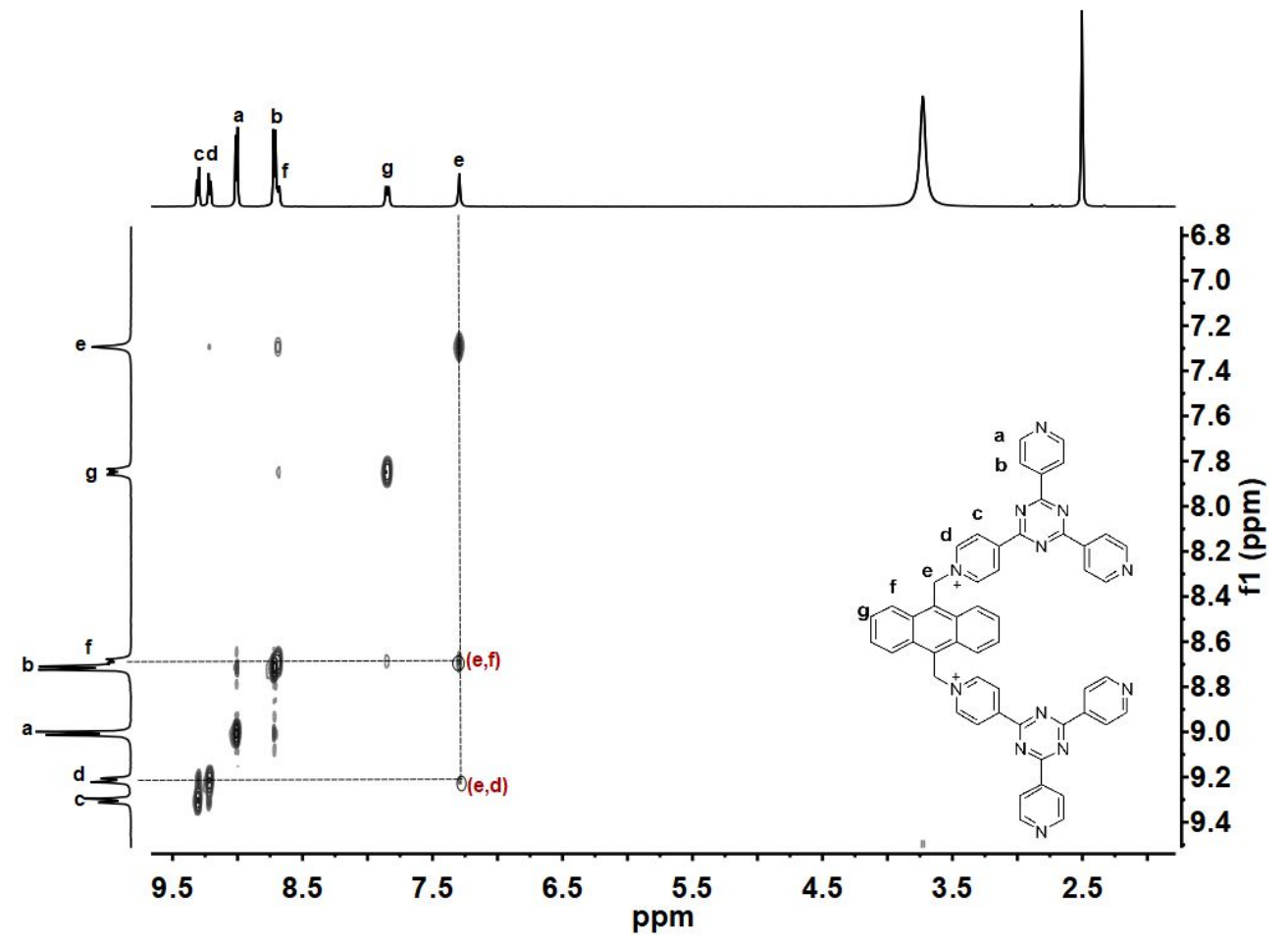

Figure S4. ${ }^{1} \mathrm{H}-{ }^{1} \mathrm{H}$ NOESY NMR spectrum $\left(600 \mathrm{MHz}, \mathrm{DMSO}-d_{6}, 298 \mathrm{~K}\right)$ of ligand $\mathrm{L}^{2} \cdot\left(\mathrm{BF}_{4}\right)_{2}$. 


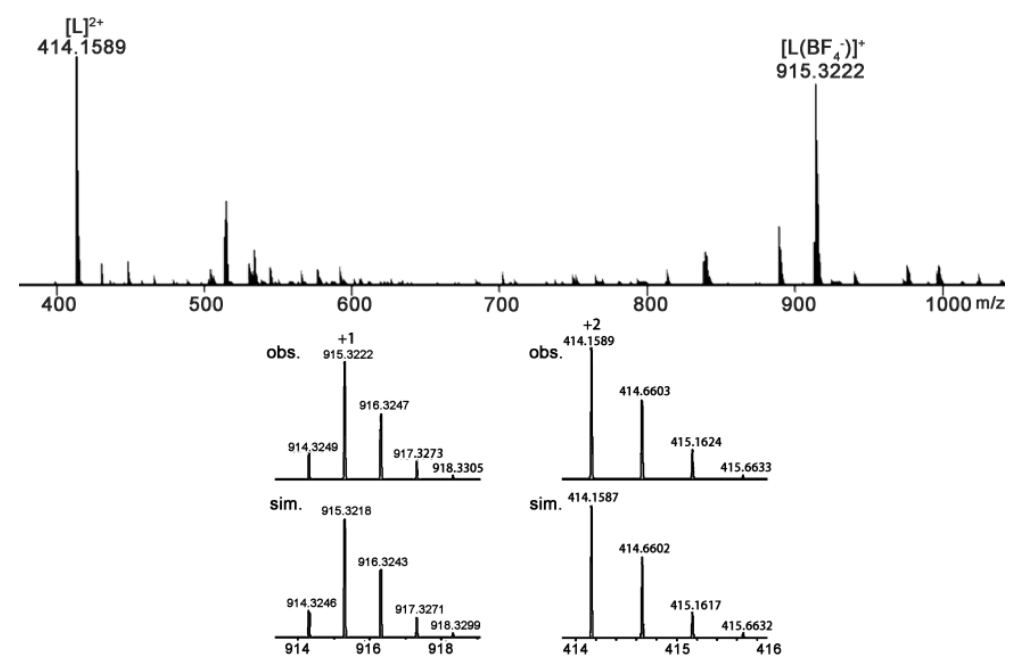

Figure S5. Top: ESI-TOF-Mass spectrum of ligand $\mathrm{L}^{2} \cdot\left(\mathrm{BF}_{4}\right)_{2}$; below: simulated and observed isotopic distribution of the $1+$ and $2+$ peaks.

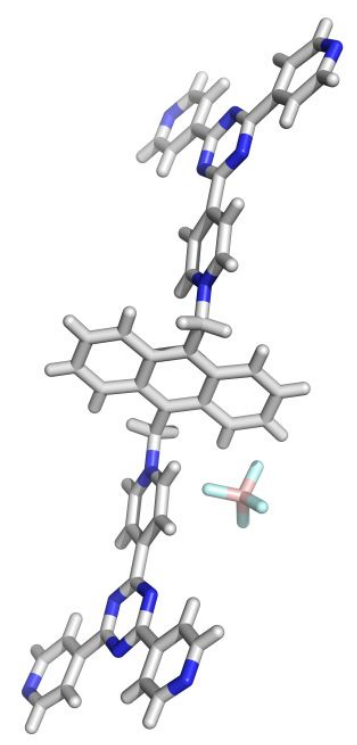

Figure S6. X-ray crystal structure of $\mathrm{L}^{2} \cdot\left(\mathrm{BF}_{4}\right)_{2}$ at a trans- conformation structure.

\subsection{Synthesis of capsule $2\left(\operatorname{Pd}_{6} \mathrm{~L}_{3}{ }_{3}\right)$}

$$
\mathrm{L}^{2}+(\mathrm{bpy}) \mathrm{Pd}\left(\mathrm{NO}_{3}\right)_{2} \underset{\mathrm{D}}{\stackrel{80^{\circ} \mathrm{C}, 12 \mathrm{~h}}{\longrightarrow}}
$$

Ligand $\mathrm{L}^{2}\left(\mathrm{BF}_{4}\right.$ salt, $\left.12.03 \mathrm{mg}, 12.00 \mu \mathrm{mol}\right)$ was treated with (bpy) $\mathrm{Pd}\left(\mathrm{NO}_{3}\right)_{2}$ $(9.27 \mathrm{mg}, 24.00 \mu \mathrm{mol})$ in $\mathrm{D}_{2} \mathrm{O}(4 \mathrm{~mL})$ at $80^{\circ} \mathrm{C}$ overnight. The reaction suspension gradually dissolved to give a homogeneous light-orange solution within $1 \mathrm{~h} .{ }^{1} \mathrm{H}$ NMR confirmed the quantitative formation of capsule $\operatorname{Pd}_{6} \mathrm{~L}_{3} \cdot\left(\mathrm{BF}_{4}\right)_{6}\left(\mathrm{NO}_{3}\right)_{12}$ $\left(\mathbf{2} \cdot\left(\mathrm{BF}_{4}\right)_{6}\left(\mathrm{NO}_{3}\right)_{12}, \mathrm{M}=5327 \mathrm{~g} / \mathrm{mol}\right)$. The solvent was removed by reduced pressure distillation to give the desired product $\left(21.0 \mathrm{mg}, 9.90 \mu \mathrm{mol}, 98.5 \%\right.$ yield). ${ }^{1} \mathrm{H}$ NMR 
$\left(600 \mathrm{MHz}, \mathrm{D}_{2} \mathrm{O}, 298 \mathrm{~K}\right): \delta 9.44(\mathrm{~d}, J=6.0 \mathrm{~Hz}, 12 \mathrm{H}), 9.40(\mathrm{~d}, J=6.0 \mathrm{~Hz}, 12 \mathrm{H}), 9.08$ $(\mathrm{d}, J=7.2 \mathrm{~Hz}, 12 \mathrm{H}), 9.00(\mathrm{~d}, J=7.2 \mathrm{~Hz}, 12 \mathrm{H}), 8.89(\mathrm{~d}, J=6.0 \mathrm{~Hz}, 12 \mathrm{H}), 8.85(\mathrm{~d}, J=$ $6.0 \mathrm{~Hz}, 12 \mathrm{H}), 8.46(\mathrm{~d}, J=8.4 \mathrm{~Hz}, 12 \mathrm{H}), 8.38(\mathrm{dd}, J=6.6,2.4 \mathrm{~Hz}, 12 \mathrm{H}), 8.35$ (t, $J=8.4$ $\mathrm{Hz}, 12 \mathrm{H}), 7.73(\mathrm{dd}, J=6.6,2.4 \mathrm{~Hz}, 12 \mathrm{H}), 7.68(\mathrm{~d}, J=4.8 \mathrm{~Hz}, 12 \mathrm{H}), 7.56(\mathrm{t}, J=$ $7.2 \mathrm{~Hz}, 12 \mathrm{H}), 7.19(\mathrm{~s}, 12 \mathrm{H}) . \mathbf{2} \cdot\left(\mathrm{BF}_{4}\right)_{12}$ was prepared by treating $\mathbf{2} \cdot\left(\mathrm{BF}_{4}\right)_{6}\left(\mathrm{NO}_{3}\right)_{12}$ with excess sodium tetrafluoroborate in an aqueous medium for electrospray ionization mass spectra (ESI-MS) measurement. ESI-TOF-MS: m/z Calcd for $\left[2-\left(\mathrm{BF}_{4}^{-}\right)_{8}\right]^{10+}$ 475.6824 found 475.6824 .

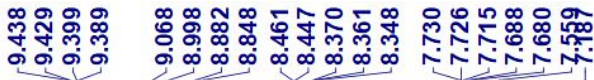

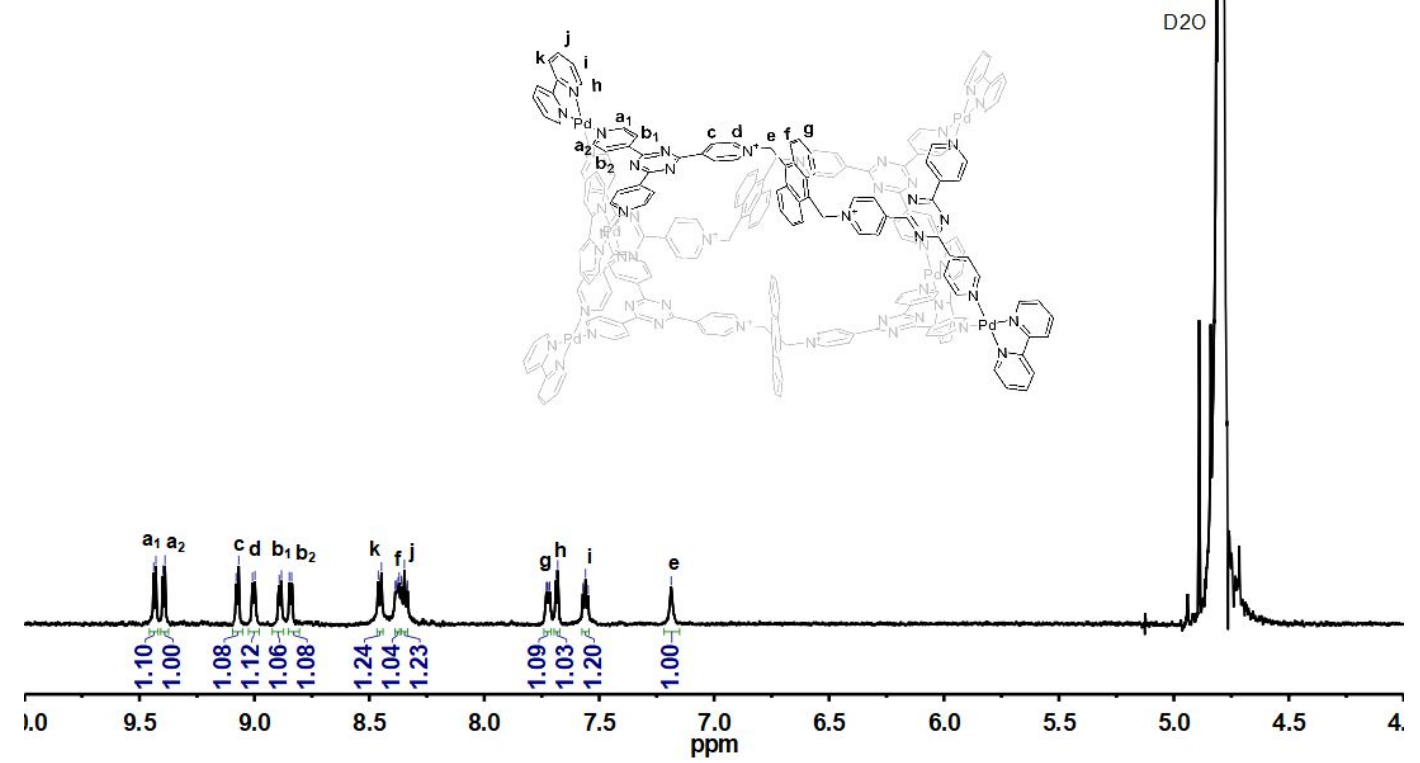

Figure S7. ${ }^{1} \mathrm{H}$ NMR spectrum $\left(600 \mathrm{MHz}, \mathrm{D}_{2} \mathrm{O}, 298 \mathrm{~K}\right)$ of capsule 2. 


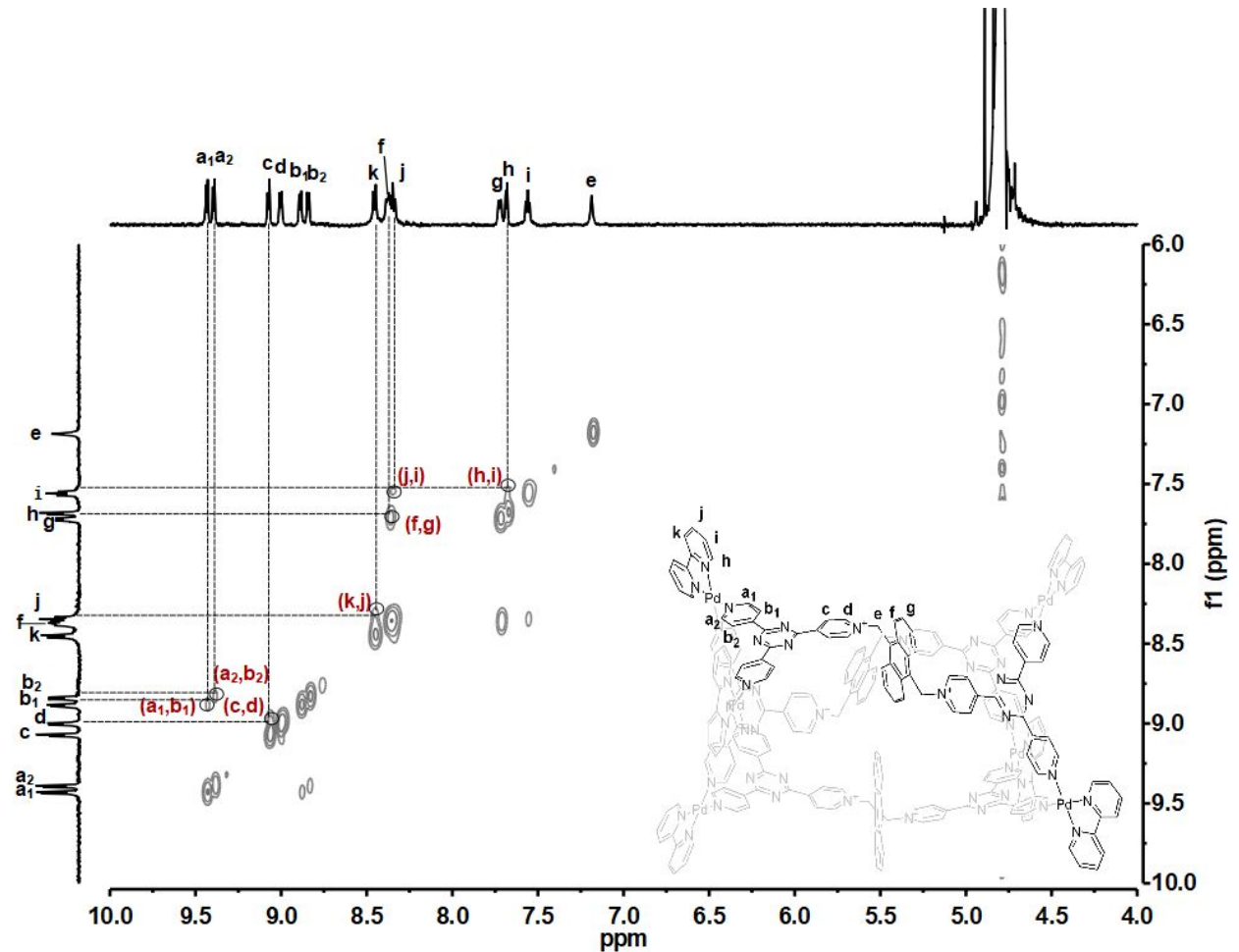

Figure S8. ${ }^{1} \mathrm{H}-{ }^{1} \mathrm{H}$ COSY NMR spectrum $\left(600 \mathrm{MHz}, \mathrm{D}_{2} \mathrm{O}, 298 \mathrm{~K}\right)$ of capsule 2.

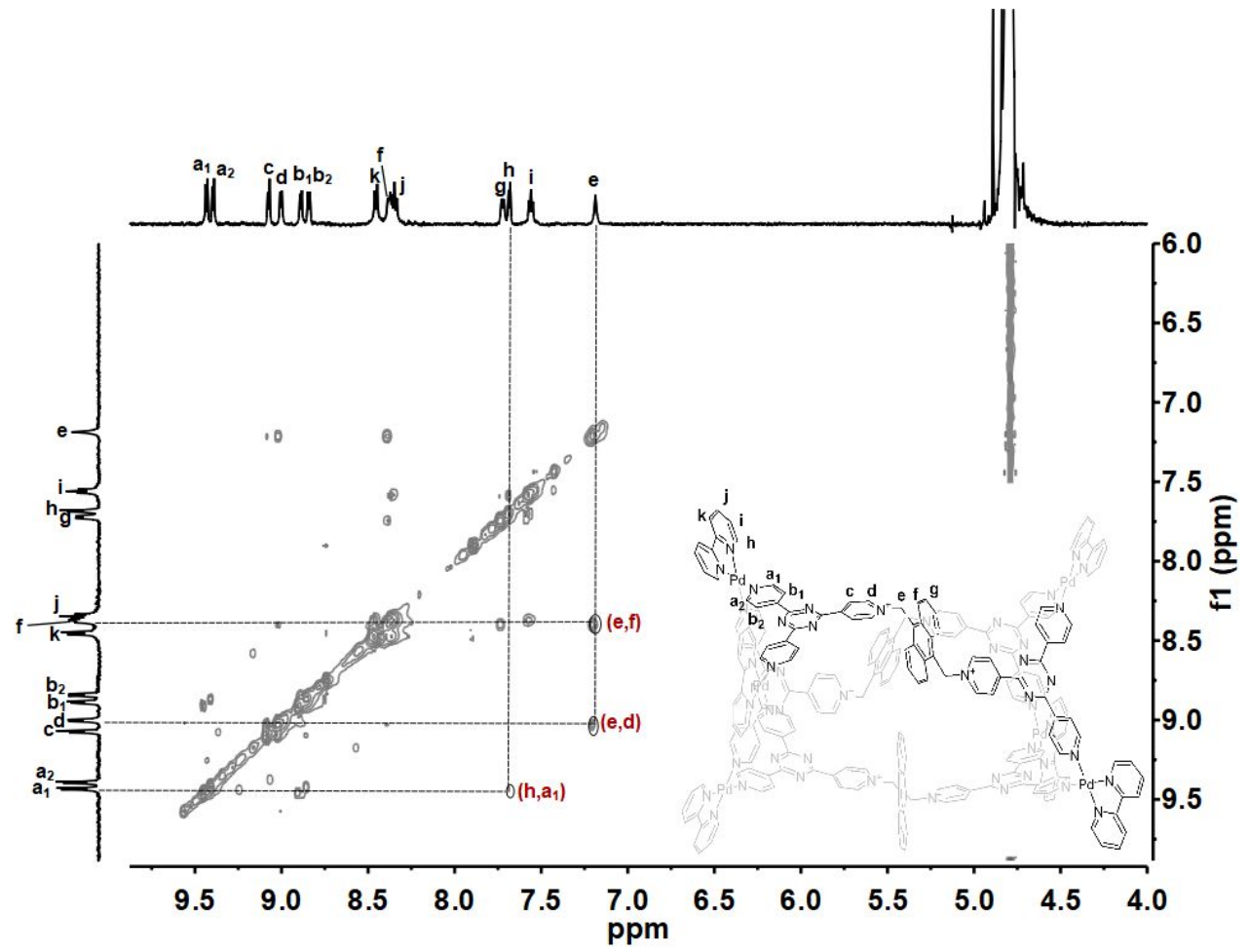

Figure S9. ${ }^{1} \mathrm{H}-{ }^{1} \mathrm{H}$ NOESY NMR spectrum $\left(600 \mathrm{MHz}, \mathrm{D}_{2} \mathrm{O}, 298 \mathrm{~K}\right)$ of capsule 2. 


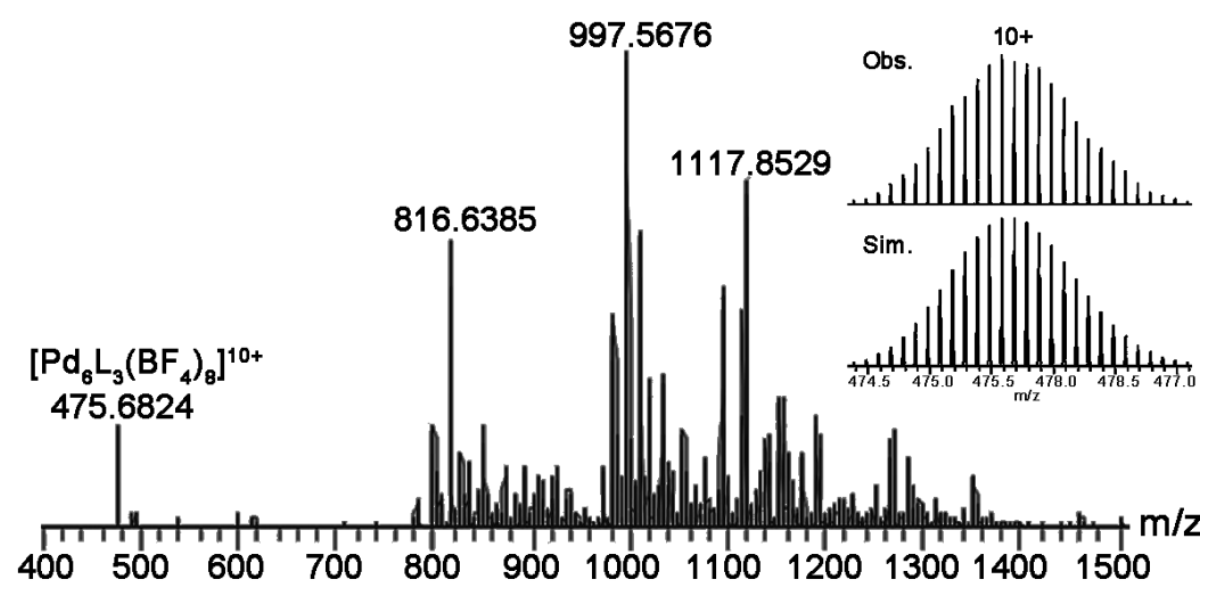

Figure S10. The ESI-TOF-Mass spectrum of capsule $2 \cdot\left(\mathrm{BF}_{4}\right)_{12}$ with inset showing the observed (Obs.) and simulated (Sim.) isotopic distribution of the $10+$ peaks.

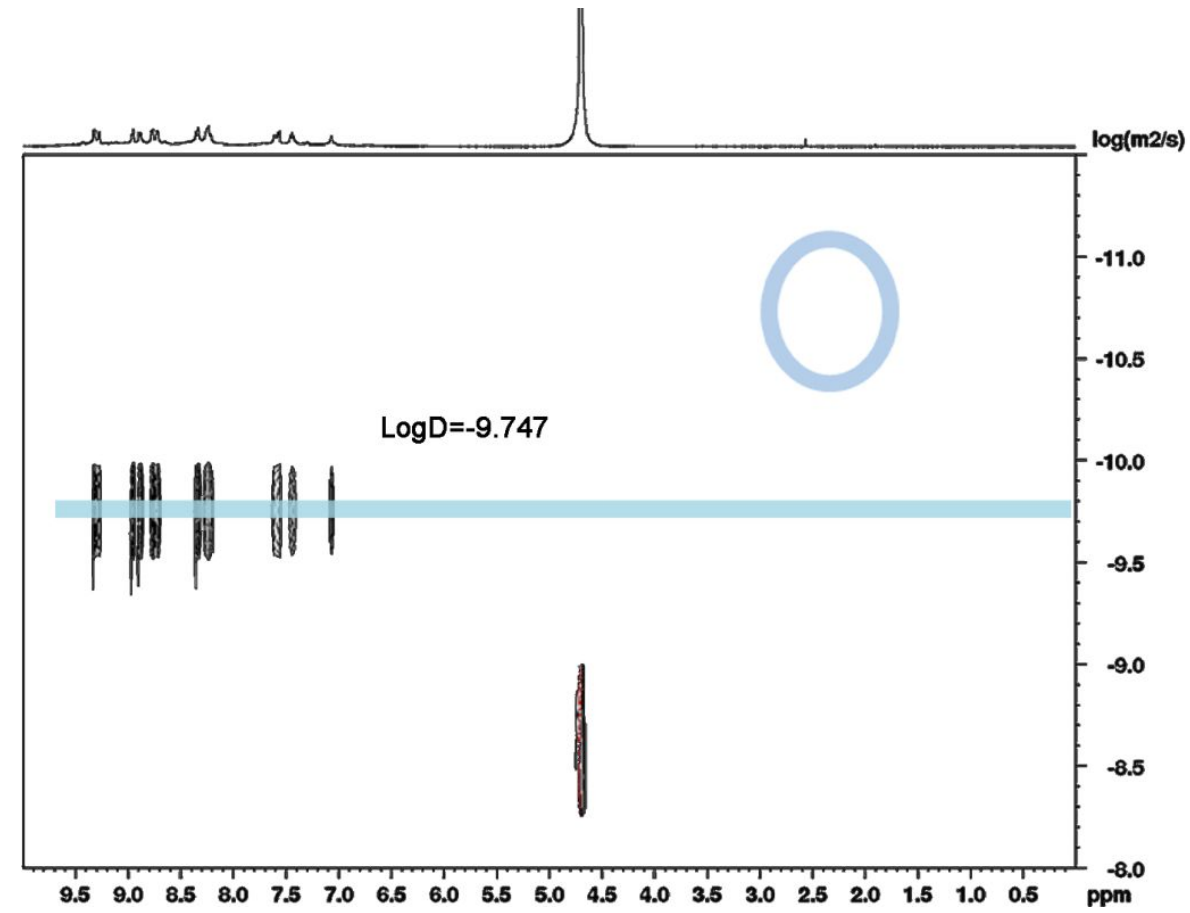

Figure S11. ${ }^{1} \mathrm{H}$ DOSY spectrum $\left(400 \mathrm{MHz}, \mathrm{D}_{2} \mathrm{O}, 298 \mathrm{~K}\right)$ of capsule 2. (Diffusion Constant $=1.79 \mathrm{E}^{-10}$ $\left.\mathrm{m}^{2} / \mathrm{S}, \mathrm{d}=2.73 \mathrm{~nm}\right)$

\section{The host-guest behavior and structure conversion of capsule 2}

\subsection{General procedure for the preparation of host-guest complexes:}

Different equiv. of guest molecules (6-20 equiv.) were added into a solution of 2 in $\mathrm{D}_{2} \mathrm{O}(5.30 \mathrm{mg}, 1 \mathrm{~mL}, 1.00 \mathrm{mM})$ until the complete formation of the host-guest complexes evidenced by ${ }^{1} \mathrm{H}$ NMR. NMR spectra are available below after filtration of the excess guests. 


\subsection{Formation of inclusion complex (adamantane derivatives) $)_{8} \subset$ capsule 2 :}

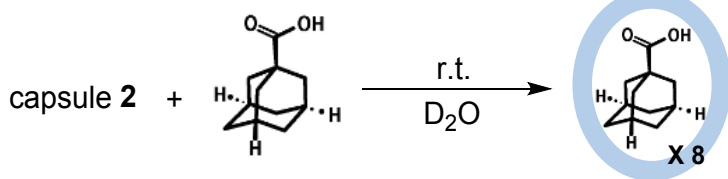

Capsule 2 (5.30 mg, $1.00 \mathrm{mmol}), \mathrm{D}_{2} \mathrm{O}(1 \mathrm{~mL}), 1$-adamantanecarboxylic acid (ADC, 10 eq., $1.80 \mathrm{mg}$ ), were added to a glass vial and then the mixture was stirred at r.t for $10 \mathrm{~min}$. Formation of the $\mathbf{A D C}_{8} \subset \mathbf{2}$ complex was confirmed based on integration ratio after filtration of the excess ADC. ${ }^{1} \mathrm{H}$ NMR $\left(400 \mathrm{MHz}, \mathrm{D}_{2} \mathrm{O}, 298 \mathrm{~K}\right)$ : $\delta 9.58(\mathrm{~d}, J=6.0 \mathrm{~Hz}, 1 \mathrm{H}), 9.54(\mathrm{~d}, J=6.0 \mathrm{~Hz}, 1 \mathrm{H}), 9.31-9.14(\mathrm{~m}, 2 \mathrm{H}), 9.04(\mathrm{~d}, J=$ $6.0 \mathrm{~Hz}, 1 \mathrm{H}), 8.97(\mathrm{~d}, J=6.0 \mathrm{~Hz}, 1 \mathrm{H}), 8.62-8.50(\mathrm{~m}, 1 \mathrm{H}), 8.48(\mathrm{~d}, J=8.4 \mathrm{~Hz}, 1 \mathrm{H})$, $8.38(\mathrm{t}, J=8.0 \mathrm{~Hz}, 1 \mathrm{H}), 7.82-7.71(\mathrm{~m}, 1 \mathrm{H}), 7.66(\mathrm{~d}, J=5.6 \mathrm{hz}, 1 \mathrm{H}), 7.58(\mathrm{t}, J=6.8 \mathrm{~Hz}$, $1 \mathrm{H}), 7.30(\mathrm{~s}, 1 \mathrm{H}), 0.91-0.24(\mathrm{~m}, 10 \mathrm{H})$.

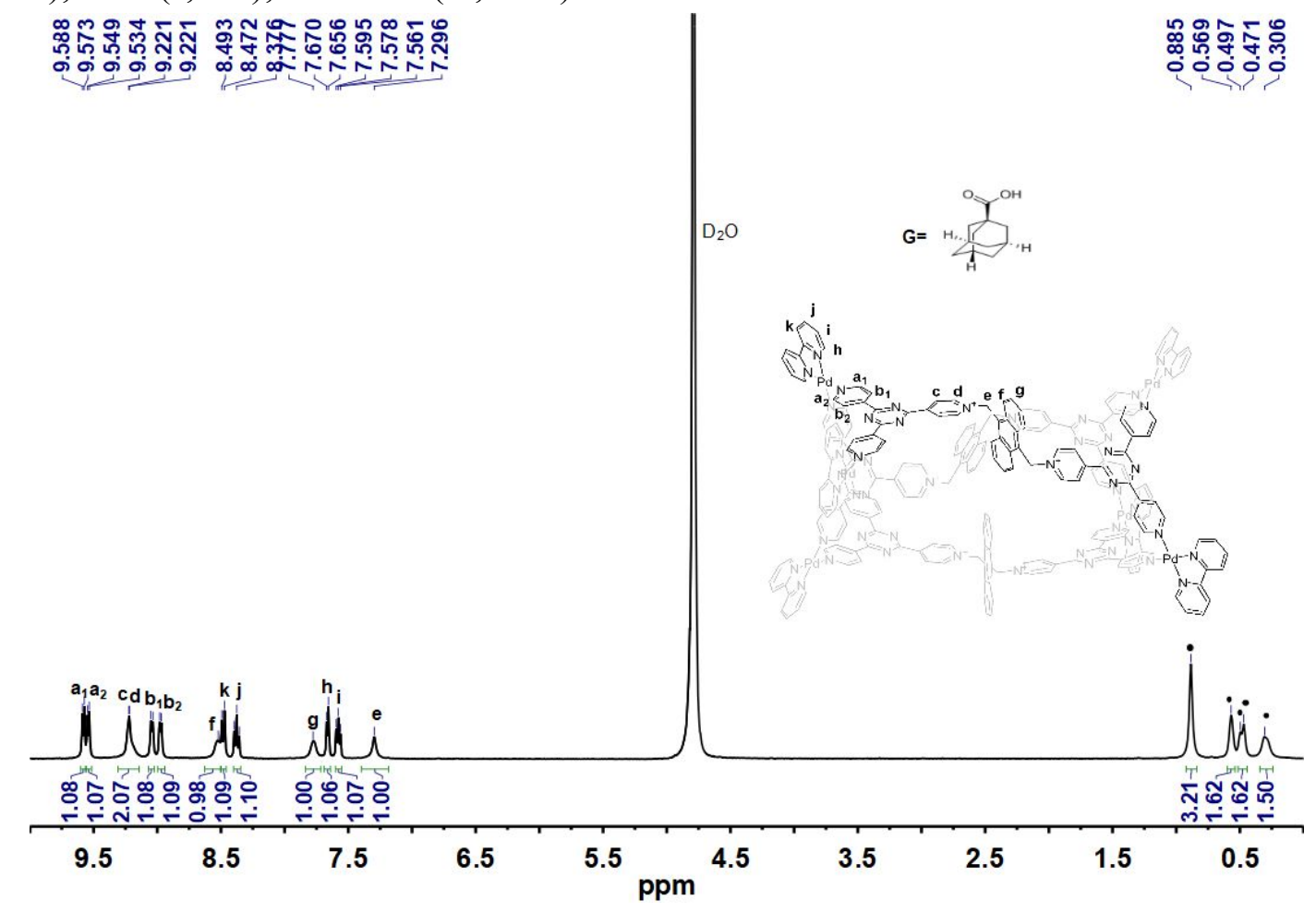

Figure S12. ${ }^{1} \mathrm{H}$ NMR spectrum $\left(400 \mathrm{MHz}, \mathrm{D}_{2} \mathrm{O}, 298 \mathrm{~K}\right)$ of $\mathbf{A D C}_{8} \subset$ capsule $2 .(\bullet=$ Guest $)$ 


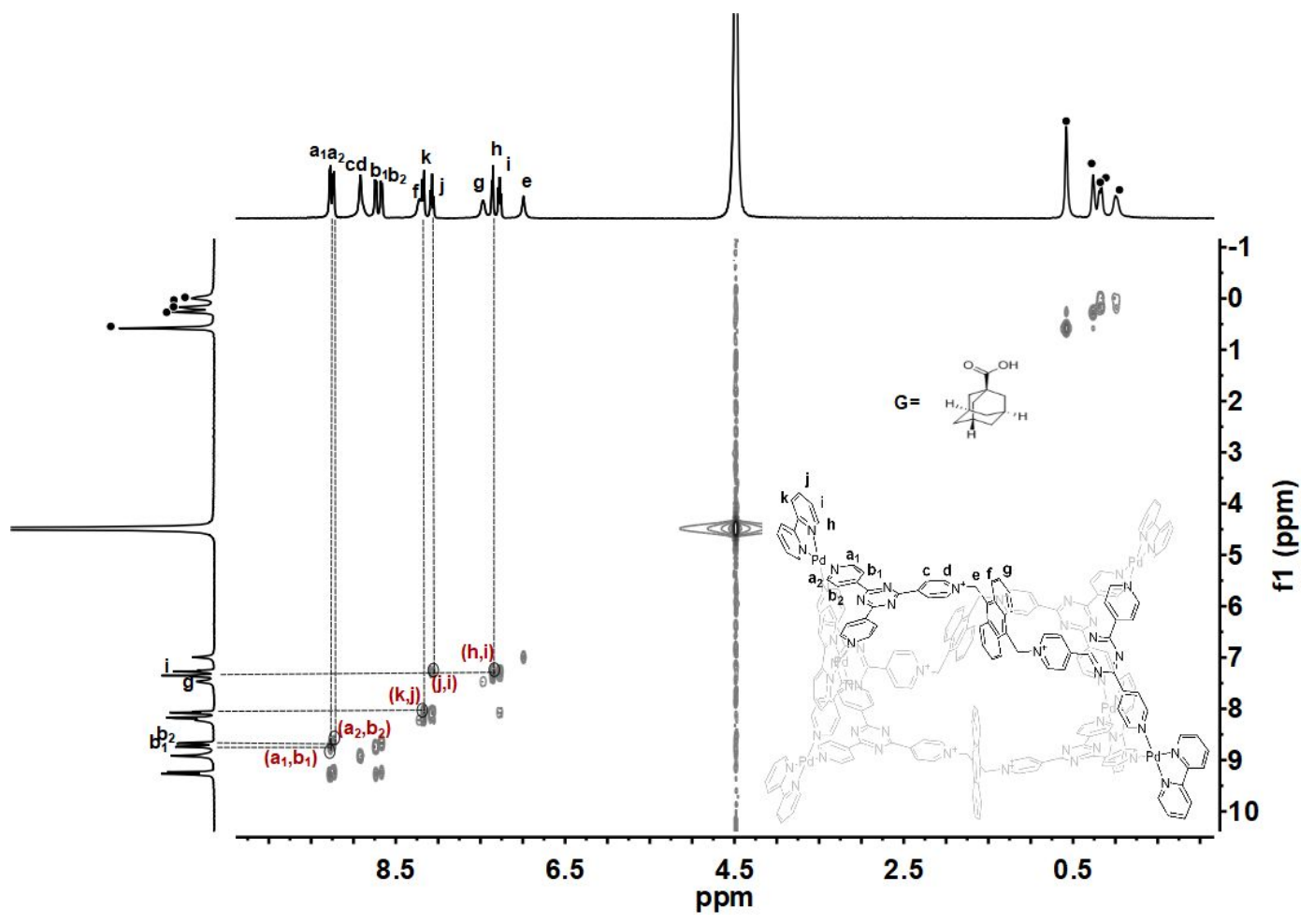

Figure S13. ${ }^{1} \mathrm{H}-{ }^{1} \mathrm{H}$ COSY NMR spectrum $\left(400 \mathrm{MHz}, \mathrm{D}_{2} \mathrm{O}, 298 \mathrm{~K}\right)$ of $\mathbf{A D C}_{8} \subset$ capsule 2. (• $=$ Guest)

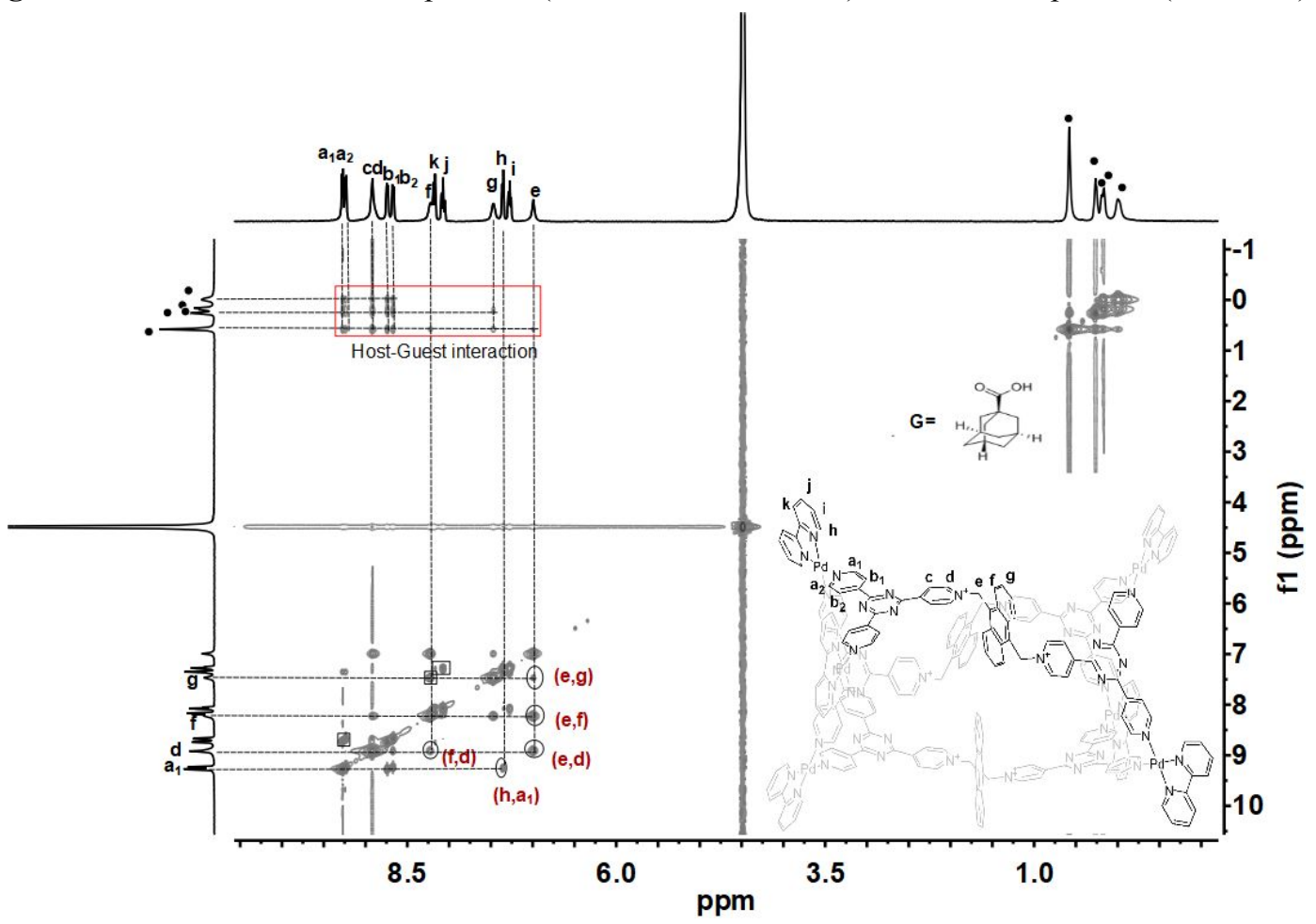

Figure S14. ${ }^{1} \mathrm{H}-{ }^{1} \mathrm{H}$ NOESY NMR spectrum $\left(400 \mathrm{MHz}, \mathrm{D}_{2} \mathrm{O}, 298 \mathrm{~K}\right)$ of $\mathbf{A D C}_{8} \subset$ capsule $\mathbf{2} .(\bullet=$ Guest $)$ 


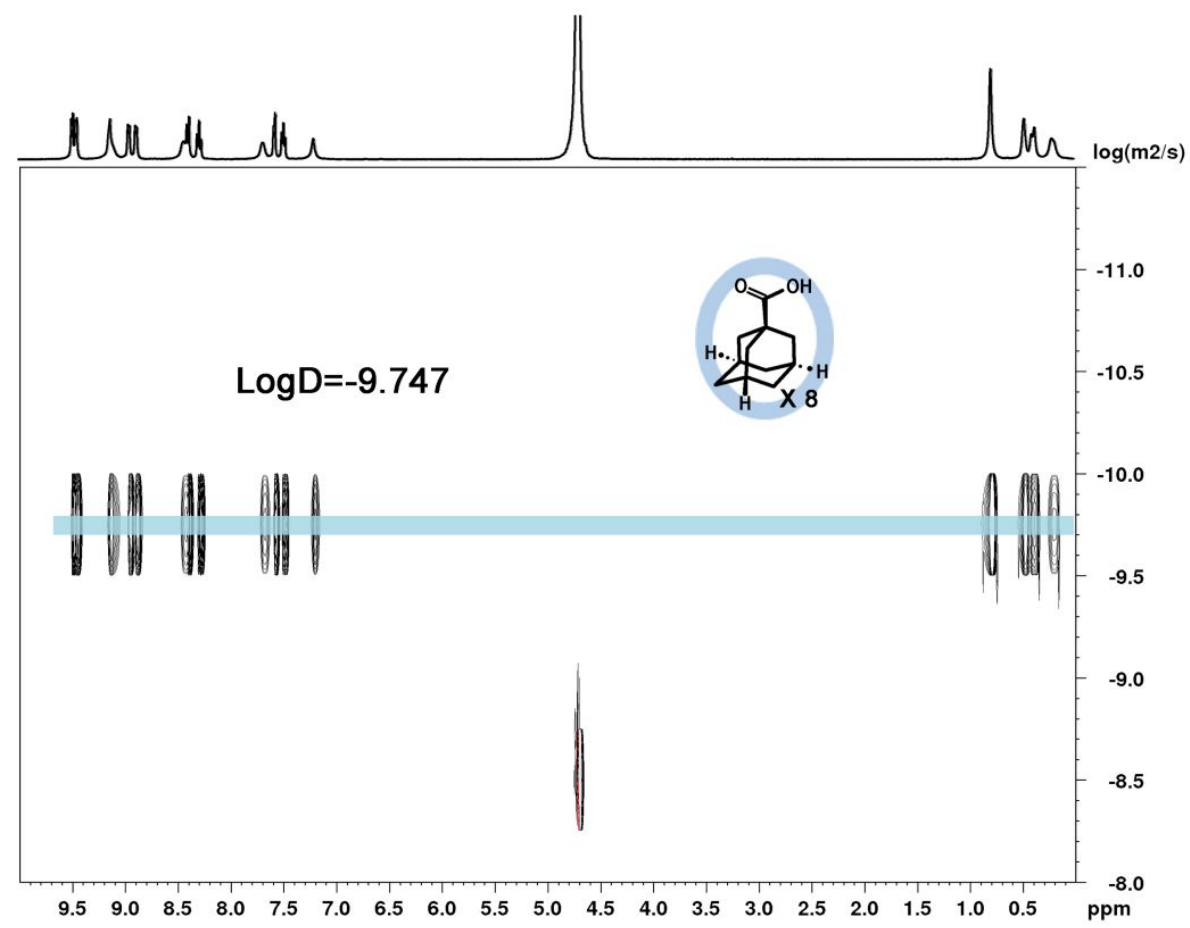

Figure S15. ${ }^{1} \mathrm{H}$ DOSY spectrum $\left(400 \mathrm{MHz}, \mathrm{D}_{2} \mathrm{O}, 298 \mathrm{~K}\right)$ of $\mathbf{A D C}_{8} \subset$ capsule 2. (Diffusion Constant $=$ $\left.1.76 \mathrm{E}^{-10} \mathrm{~m}^{2} / \mathrm{S}, \mathrm{d}=2.73 \mathrm{~nm}\right)$

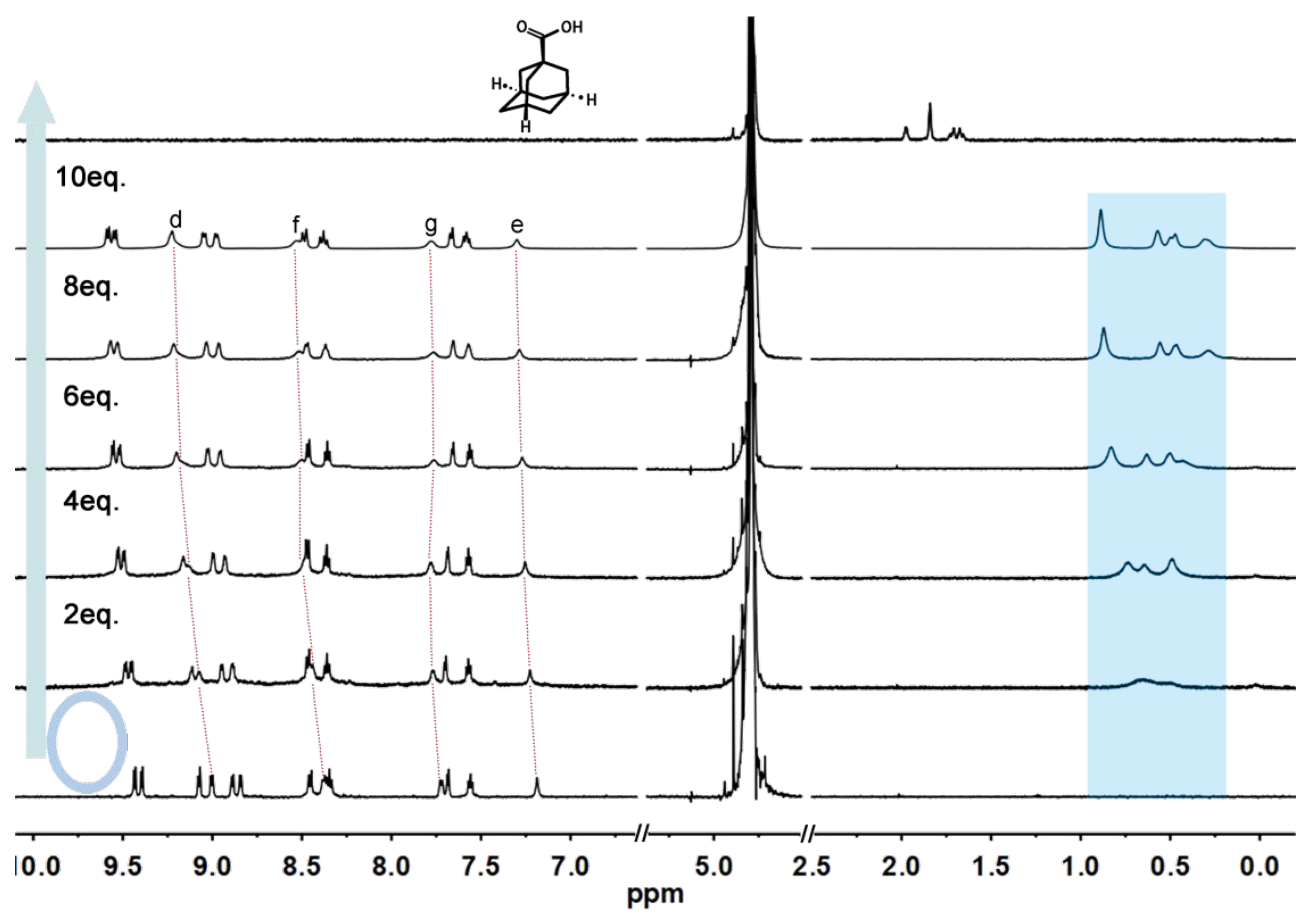

Figure S16. ${ }^{1} \mathrm{H}$ NMR spectra $\left(600 \mathrm{M} \mathrm{Hz}, \mathrm{D}_{2} \mathrm{O}, 298 \mathrm{~K}\right)$ of capsule 2 when titrated with ADC (0-10 eq.), indicative of fast exchange on the NMR timescale.

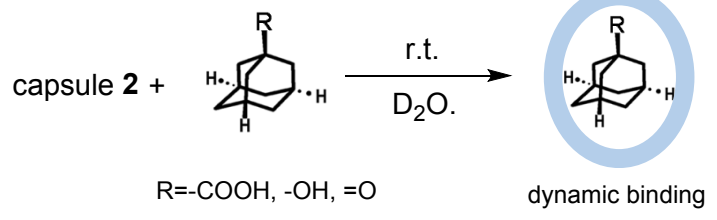




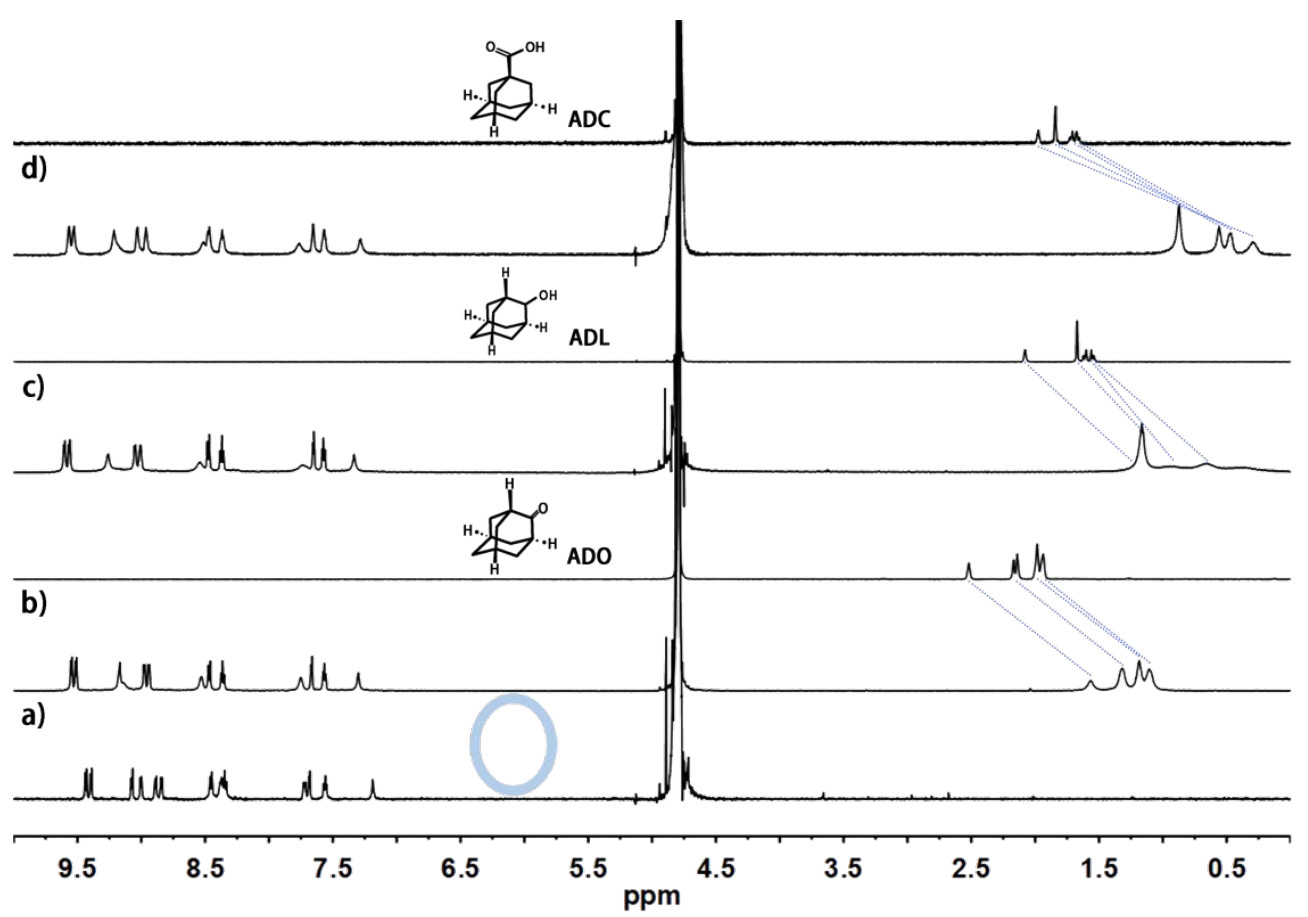

Figure S17. ${ }^{1} \mathrm{H}$ NMR spectra $\left(600 \mathrm{MHz}, \mathrm{D}_{2} \mathrm{O}, 298 \mathrm{~K}\right)$ of capsule 2 and capsule 2 after adding excess adamantane derivatives (ADC, ADL, ADO) as guests, showing the obvious upshifts of guest signals.

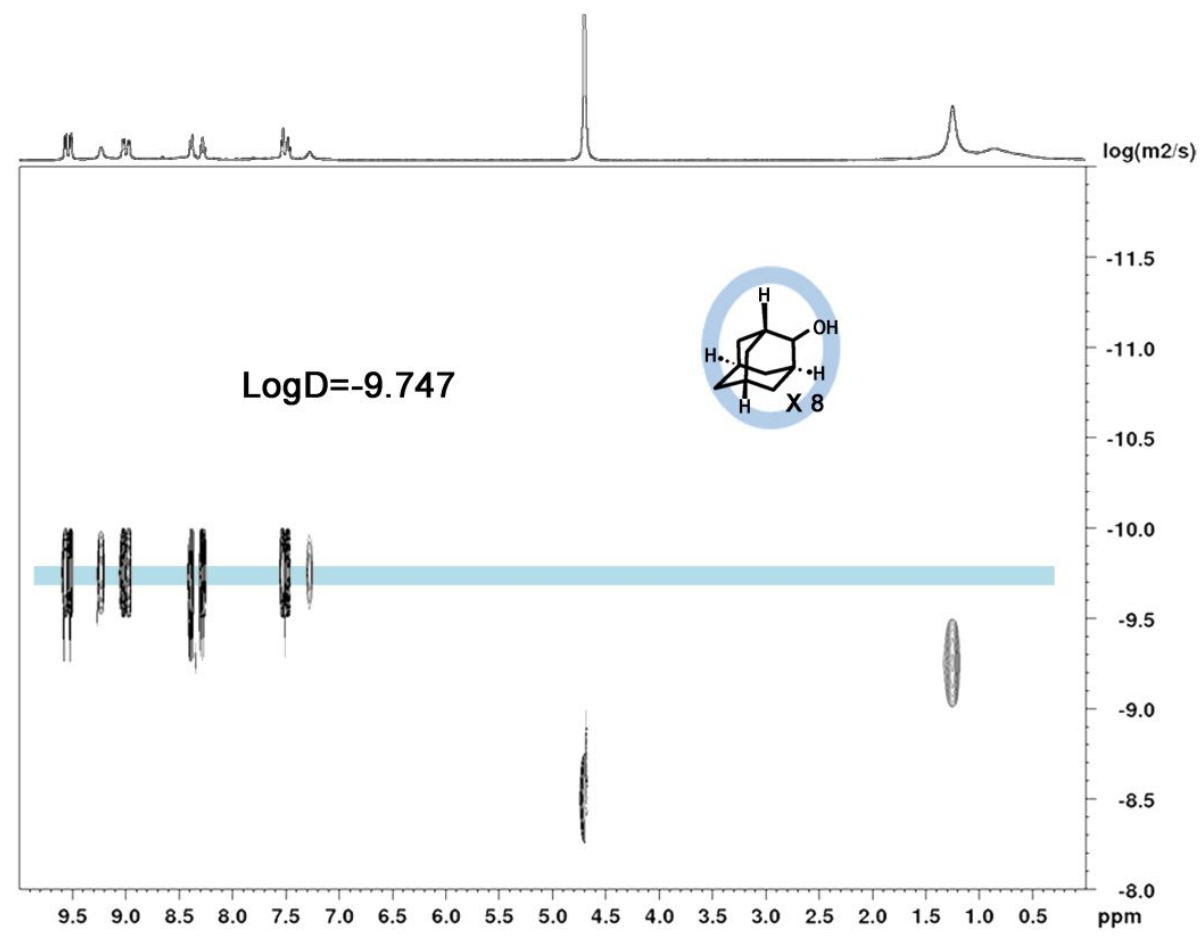

Figure S18. ${ }^{1} \mathrm{H}$ DOSY spectrum $\left(400 \mathrm{MHz}, \mathrm{D}_{2} \mathrm{O}, 298 \mathrm{~K}\right)$ of $\mathbf{A D L}_{8} \subset$ capsule 2. (Diffusion Constant $=$ $\left.1.76 \mathrm{E}^{-10} \mathrm{~m}^{2} / \mathrm{S}, \mathrm{d}=2.73 \mathrm{~nm}\right)$ 


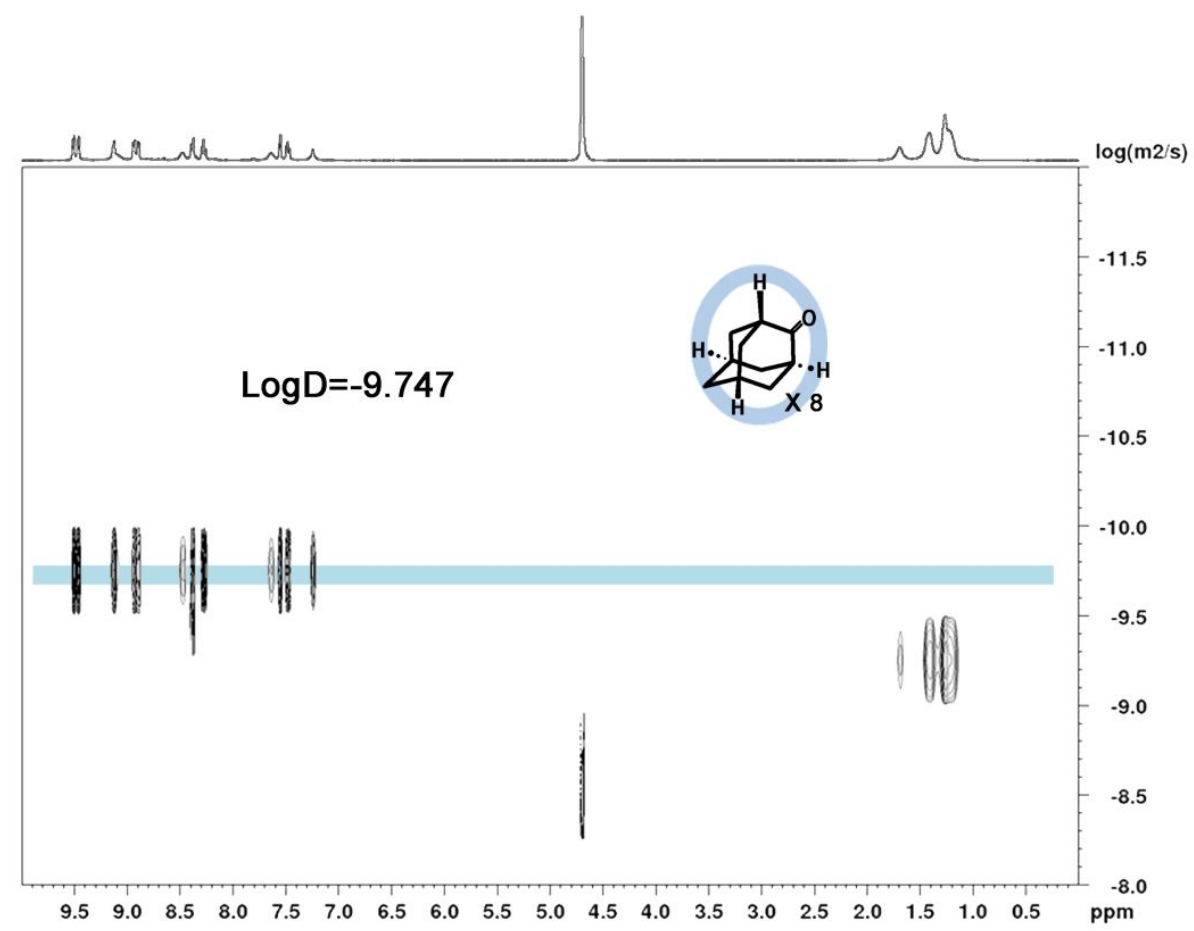

Figure S19. ${ }^{1} \mathrm{H}$ DOSY spectrum $\left(400 \mathrm{MHz}, \mathrm{D}_{2} \mathrm{O}, 298 \mathrm{~K}\right)$ of $\mathbf{A D O}_{8} \subset$ capsule 2 . (Diffusion Constant $=$ $\left.1.76 \mathrm{E}^{-10} \mathrm{~m}^{2} / \mathrm{S}, \mathrm{d}=2.73 \mathrm{~nm}\right)$

\subsection{Formation of inclusion complex (methyl(4-nitrophenyl)sulfane) ${ }_{3} \subset$ bowl 3:}

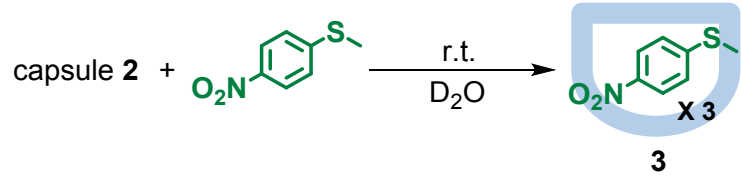

Capsule 2 (5.30 mg, $1.00 \mathrm{mmol}), \mathrm{D}_{2} \mathrm{O}(1 \mathrm{~mL})$, methyl(4-nitrophenyl)sulfane (MNS, $1.70 \mathrm{mg}, 10$ eq.), were added to a glass vial and then the mixture was stirred at r.t for $10 \mathrm{~min}$. The formation of $\mathbf{M N S}_{3} \subset \mathbf{3}$ complex was confirmed based on the integration ratio after filtration of the excess MNS. ${ }^{1} \mathrm{H}$ NMR $\left(400 \mathrm{MHz}, \mathrm{D}_{2} \mathrm{O}, 298 \mathrm{~K}\right)$ : $\delta 9.64(\mathrm{~d}, J=6.0 \mathrm{~Hz}, 1 \mathrm{H}), 9.55-9.45(\mathrm{~m}, 2 \mathrm{H}), 9.35(\mathrm{~d}, J=6.0 \mathrm{~Hz}, 1 \mathrm{H}), 9.06-8.95(\mathrm{~m}$, $3 \mathrm{H}), 8.89(\mathrm{~d}, J=6.0 \mathrm{~Hz}, 2 \mathrm{H}), 8.85(\mathrm{~d}, J=6.0 \mathrm{~Hz}, 1 \mathrm{H}), 8.59-8.53(\mathrm{~m}, 1 \mathrm{H}), 8.52-8.45$ $(\mathrm{m}, 3 \mathrm{H}), 8.44(\mathrm{~d}, J=6.0 \mathrm{~Hz}, 1 \mathrm{H}), 8.37(\mathrm{t}, J=8.0 \mathrm{~Hz}, 2 \mathrm{H}), 8.12-8.04(\mathrm{~m}, 1 \mathrm{H})$, 7.88-7,81 (m, 1H), $7.74(\mathrm{~d}, J=4.0 \mathrm{~Hz}, 1 \mathrm{H}), 7.62-7.50(\mathrm{~m}, 3 \mathrm{H}), 7.35-7.26(\mathrm{~m}, 1 \mathrm{H})$, $7.22(\mathrm{~d}, J=15.2 \mathrm{~Hz}, 1 \mathrm{H}), 6.95(\mathrm{~d}, J=15.2 \mathrm{~Hz}, 1 \mathrm{H}), 6.23-6.12(\mathrm{~m}, 2 \mathrm{H}), 5.43(\mathrm{~m}, 2 \mathrm{H})$, $1.19(\mathrm{~s}, 2 \mathrm{H})$. 


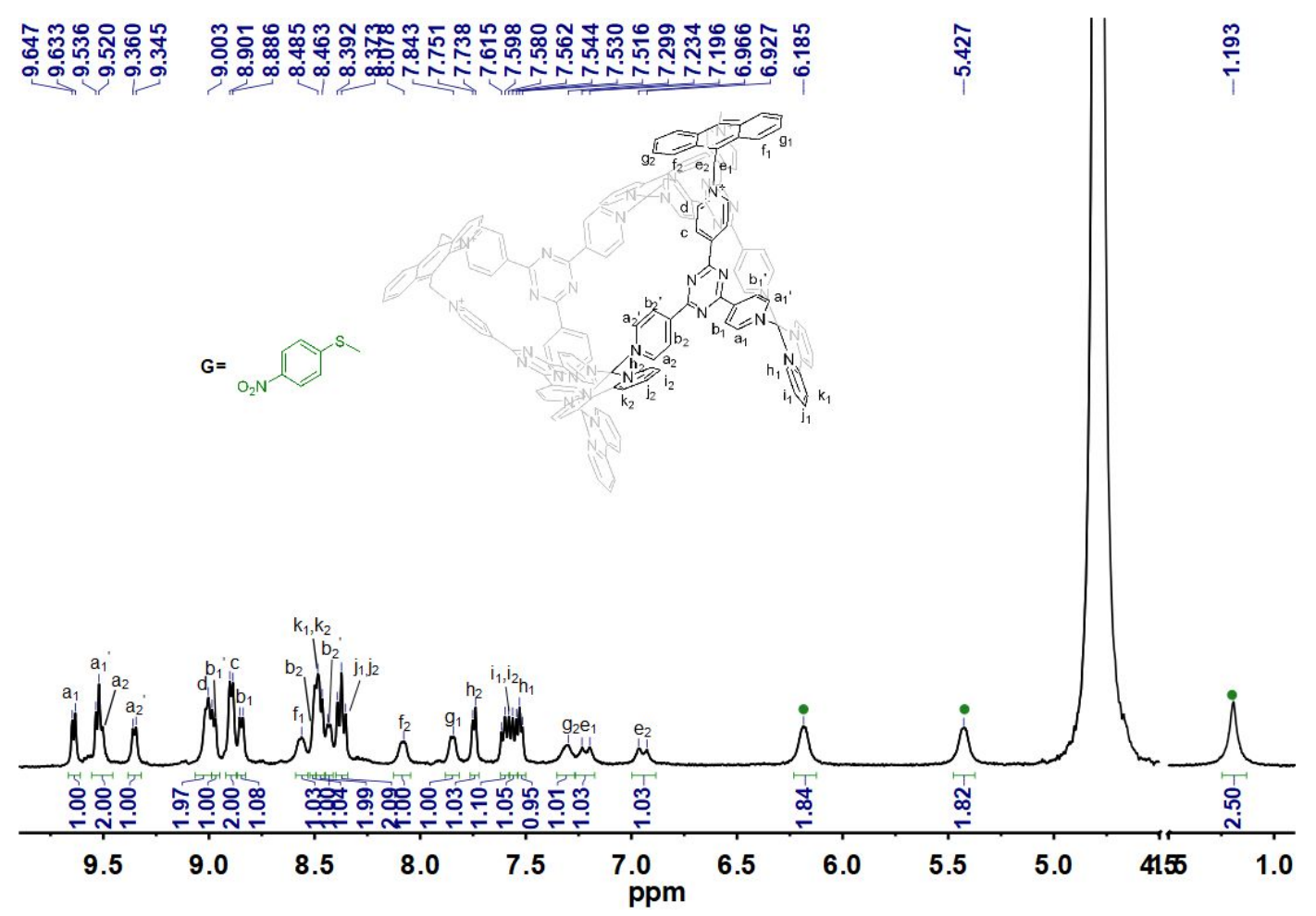

Figure S20. ${ }^{1} \mathrm{H}$ NMR spectrum $\left(400 \mathrm{MHz}, \mathrm{D}_{2} \mathrm{O}, 298 \mathrm{~K}\right)$ of $\mathbf{M N S}_{3} \subset$ bowl 3 transformed from capsule 2. $(\bullet=$ Guest $)$

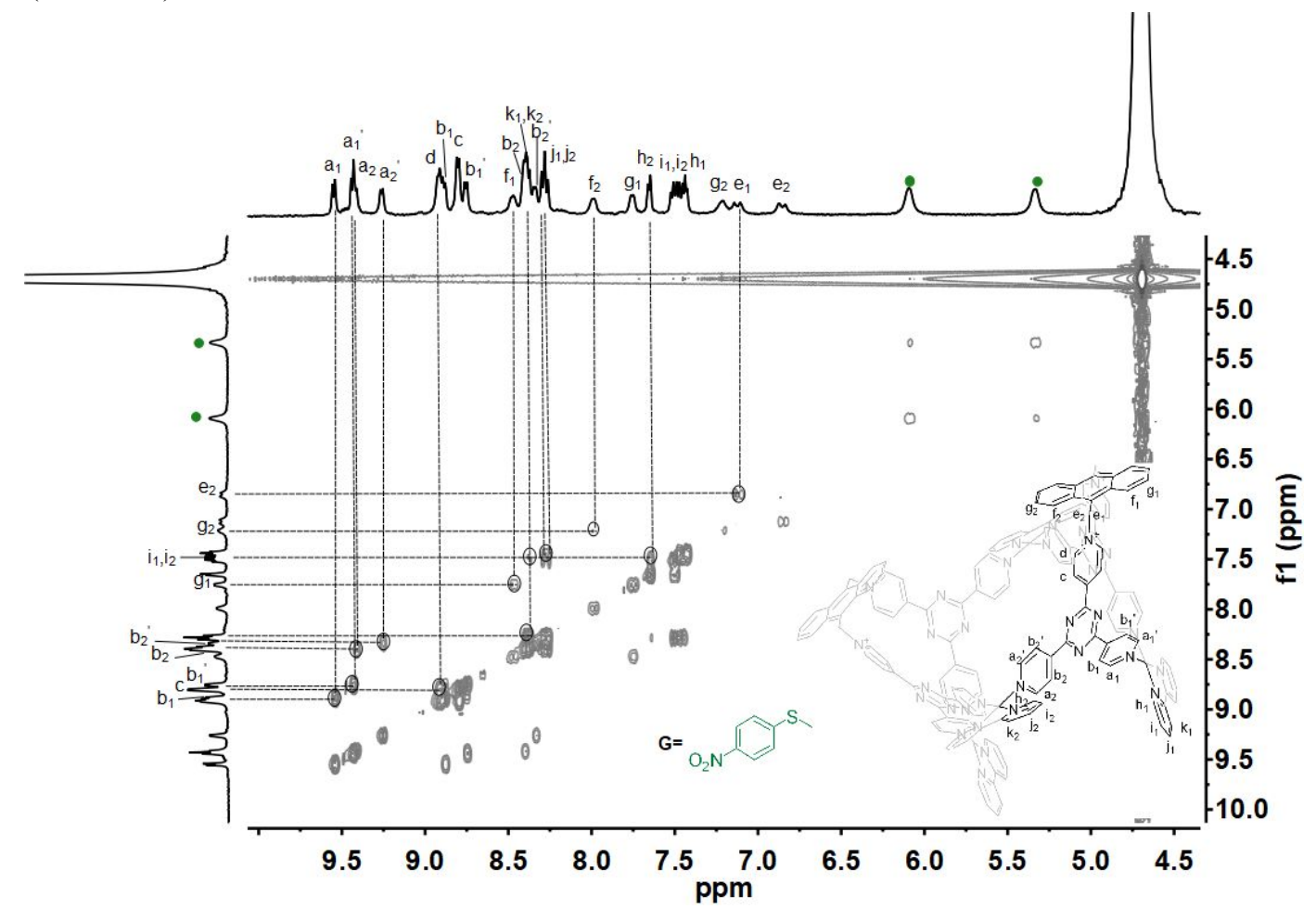

Figure S21. ${ }^{1} \mathrm{H}-{ }^{1} \mathrm{H}$ COSY NMR spectrum (400 MHz, $\left.\mathrm{D}_{2} \mathrm{O}, 298 \mathrm{~K}\right)$ of $\mathbf{M N S}_{3} \subset$ bowl 3 transformed from capsule 2 . $(\bullet=$ Guest $)$ 


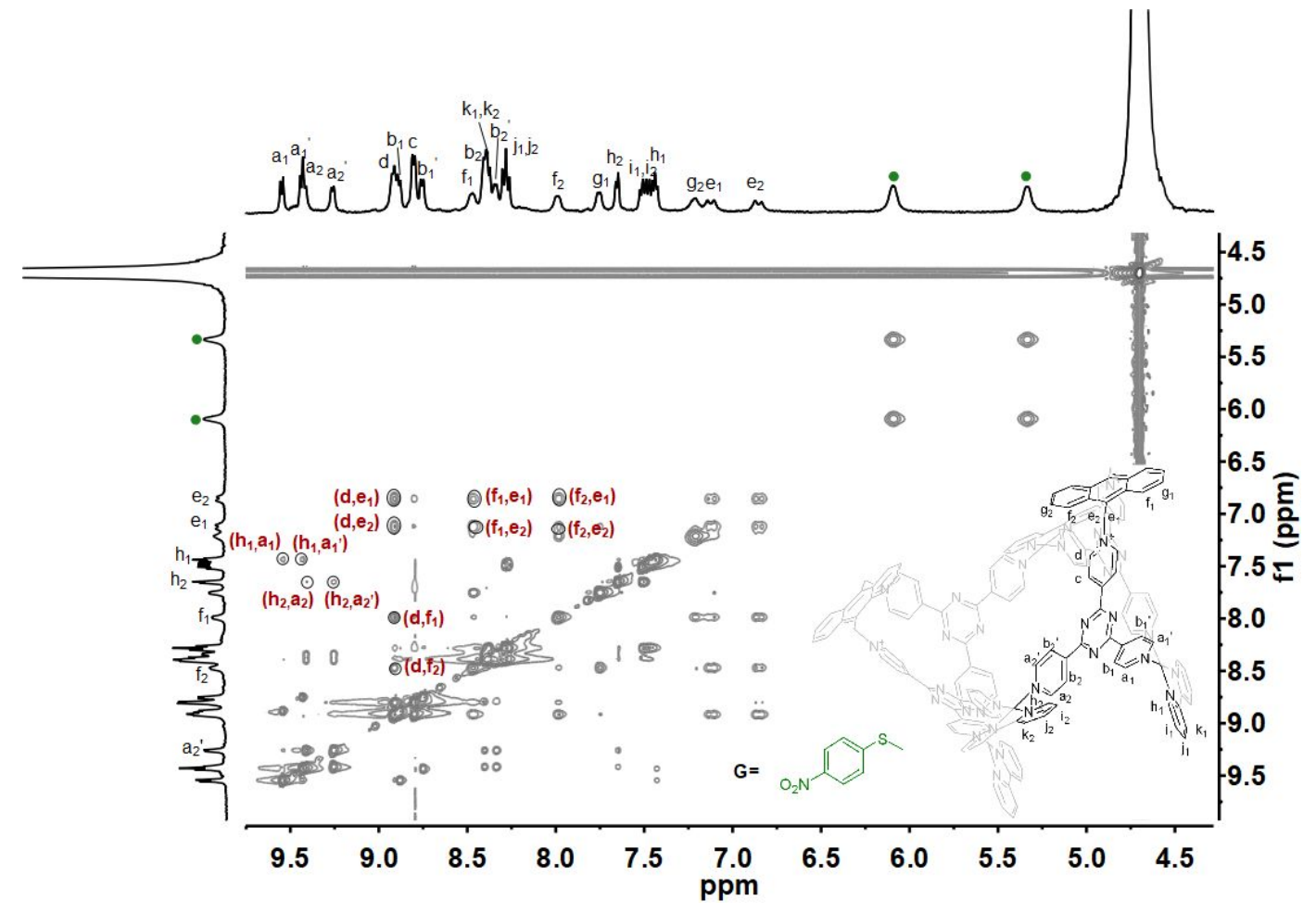

Figure S22. ${ }^{1} \mathrm{H}-{ }^{1} \mathrm{H}$ NOESY spectrum $\left(400 \mathrm{MHz}, \mathrm{D}_{2} \mathrm{O}, 298 \mathrm{~K}\right)$ of $\mathbf{M N S}_{3} \subset$ bowl 3 transformed from capsule 2. $(\bullet=$ Guest $)$

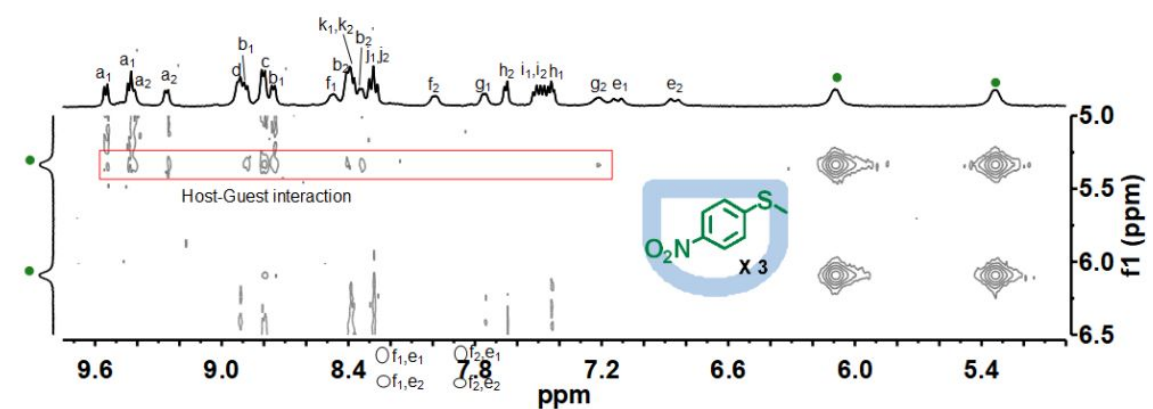

Figure S23. Partial ${ }^{1} \mathrm{H}-{ }^{-1} \mathrm{H}$ NOESY NMR spectrum $\left(400 \mathrm{MHz}, \mathrm{D}_{2} \mathrm{O}, 298 \mathrm{~K}\right)$ of $\mathbf{M N S}_{3} \subset$ bowl $\mathbf{3}$ showing NOE correlations between the guests and TPT panel on bowl 3.(the corresponding full-range spectrum is shown in Fig.S22). ( $\bullet$ Guest $)$ 


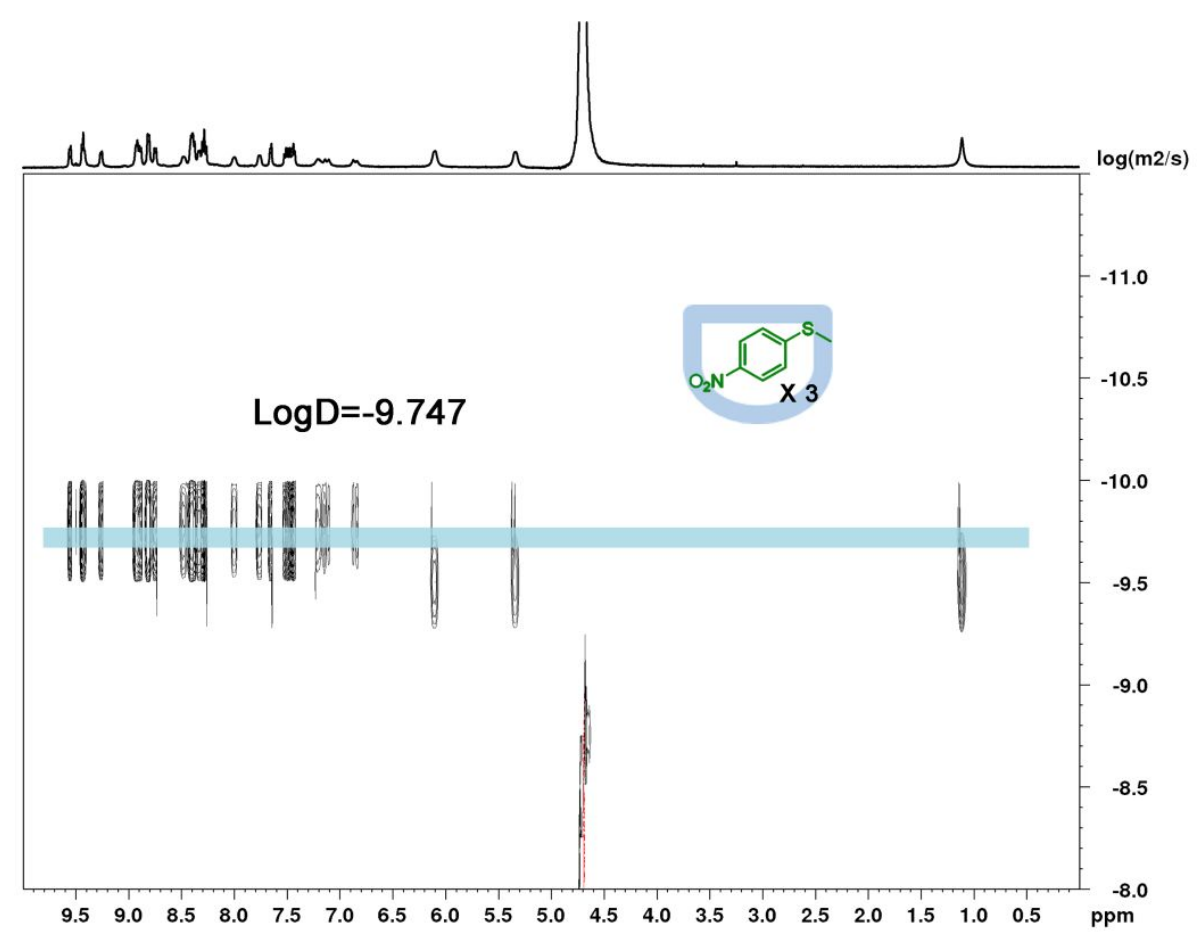

Figure S24. ${ }^{1} \mathrm{H}$ DOSY spectrum $\left(400 \mathrm{MHz}, \mathrm{D}_{2} \mathrm{O}, 298 \mathrm{~K}\right)$ of $\mathbf{M N S}_{3} \subset$ bowl 3 transformed from capsule 2. (Diffusion Constant $=1.79 \mathrm{E}^{-10} \mathrm{~m}^{2} / \mathrm{S}, \mathrm{d}=2.73 \mathrm{~nm}$ )

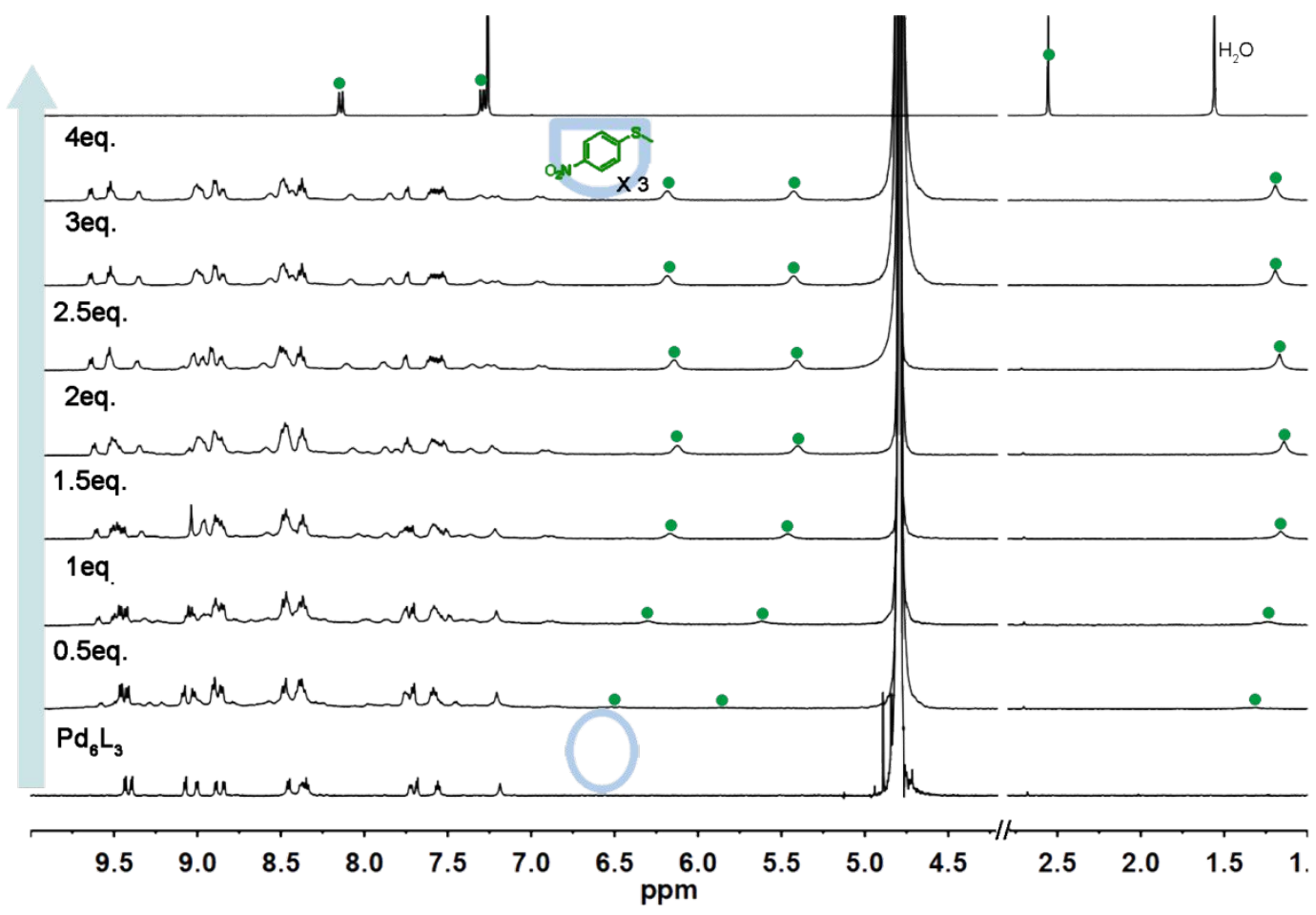

Figure S25. ${ }^{1} \mathrm{H}$ NMR spectra $\left(400 \mathrm{~Hz}, \mathrm{D}_{2} \mathrm{O}, 298 \mathrm{~K}\right)$ of capsule 2 when titrated with MNS, showing fast exchange binding kinetics on the NMR timescale $(\bullet=$ Guest $)$. A 1:3 binding stoichiometry was feasible based on the crystal structure (see the crystal data section for details). 
4.4 Formation of inclusion complex (naphthalene) ${ }_{3} \subset$ bowl 3:

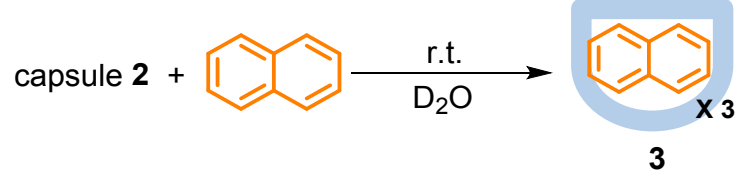

Capsule 2 (5.30 mg, $1.00 \mathrm{mmol}), \mathrm{D}_{2} \mathrm{O}(1 \mathrm{~mL})$, naphthalene (NAP, 10 eq., 1.28 $\mathrm{mg}$ ), were added to a glass vial and then the mixture was stirred at r.t. for $10 \mathrm{~min}$. The formation of $\mathbf{N A P}_{\mathbf{3}} \subset \mathbf{3}$ complex was confirmed based on the integration ratio after filtration of the excess NAP. ${ }^{1} \mathrm{H}$ NMR $\left(400 \mathrm{MHz}, \mathrm{D}_{2} \mathrm{O}, 298 \mathrm{~K}\right): \delta 9.70(\mathrm{~d}, J=6.0 \mathrm{~Hz}$, $1 \mathrm{H}), 9.65(\mathrm{~d}, J=6.0 \mathrm{~Hz}, 1 \mathrm{H}), 9.60(\mathrm{~d}, J=6.0 \mathrm{~Hz}, 1 \mathrm{H}), 9.48(\mathrm{~d}, J=6.0 \mathrm{~Hz}, 1 \mathrm{H})$, 9.15-9.16 (m, 2H), $8.80(\mathrm{~d}, J=6.0 \mathrm{~Hz}, 1 \mathrm{H}), 8.75-8.65(\mathrm{~m}, 3 \mathrm{H}), 8.51-8.45(\mathrm{~m}, 3 \mathrm{H})$, 8.41-8.35 (m, 3H), 8.34-8.28 (m, 2H), 8.02-8.89 (m, 2H), $7.76(\mathrm{~d}, J=5.2 \mathrm{~Hz}, 1 \mathrm{H})$, $7.67(\mathrm{~d}, J=5.2 \mathrm{~Hz}, 1 \mathrm{H}), 7.64-7.53(\mathrm{~m}, 2 \mathrm{H}), 7.27(\mathrm{~d}, J=14.4 \mathrm{~Hz}, 1 \mathrm{H}), 7.10(\mathrm{~d}, J=$ $14.4 \mathrm{~Hz}, 1 \mathrm{H}), 5.47-5.40(\mathrm{~m}, 3 \mathrm{H}), 4.92-4.86(\mathrm{~m}, 3 \mathrm{H})$.

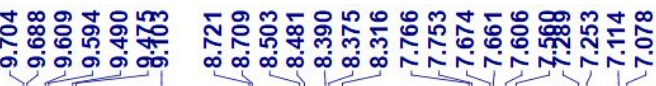

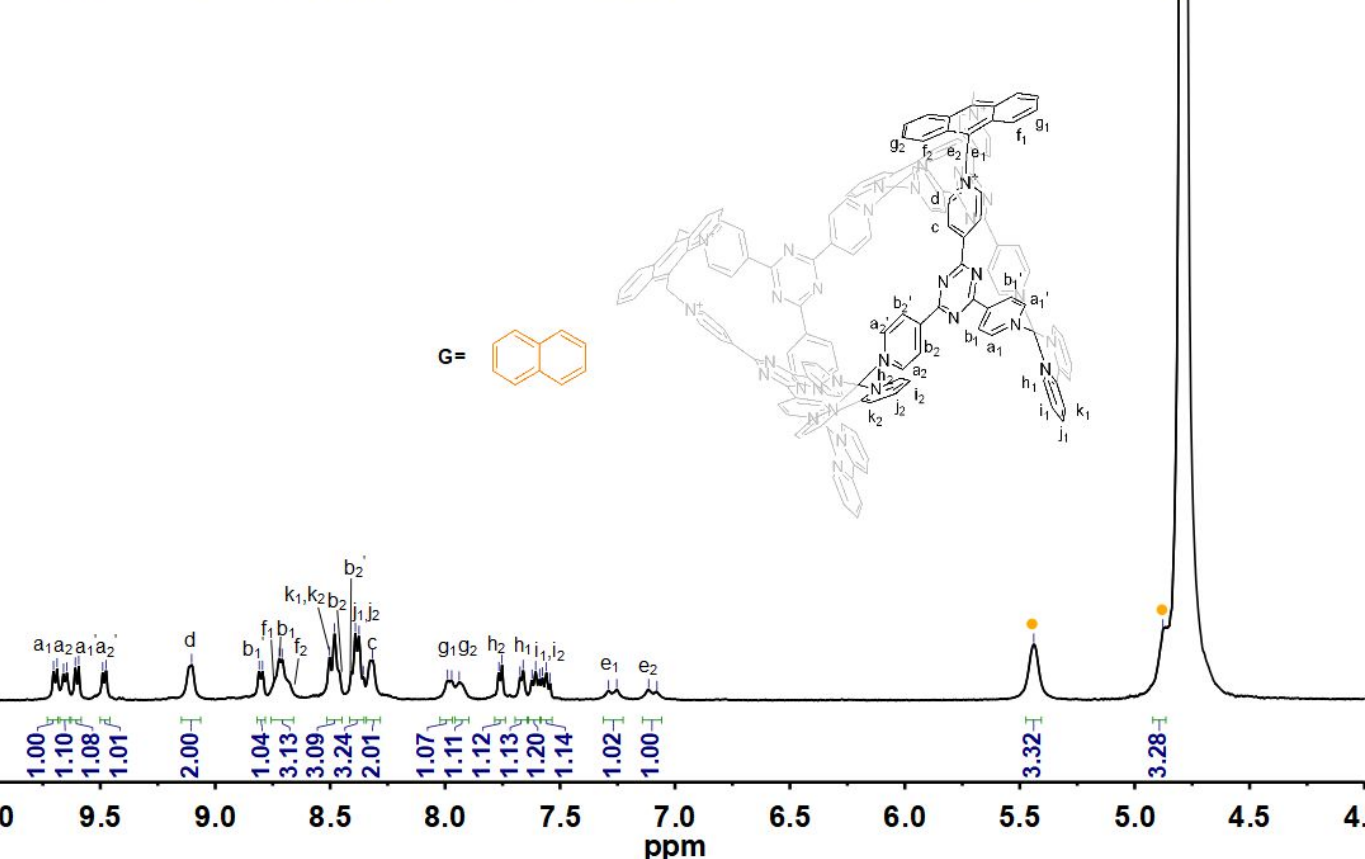

Figure S26. ${ }^{1} \mathrm{H}$ NMR spectrum $\left(400 \mathrm{MHz}, \mathrm{D}_{2} \mathrm{O}, 298 \mathrm{~K}\right)$ of $\mathbf{N A P}_{3} \subset$ bowl 3 transformed from capsule 2. $(\bullet=$ Guest $)$ 


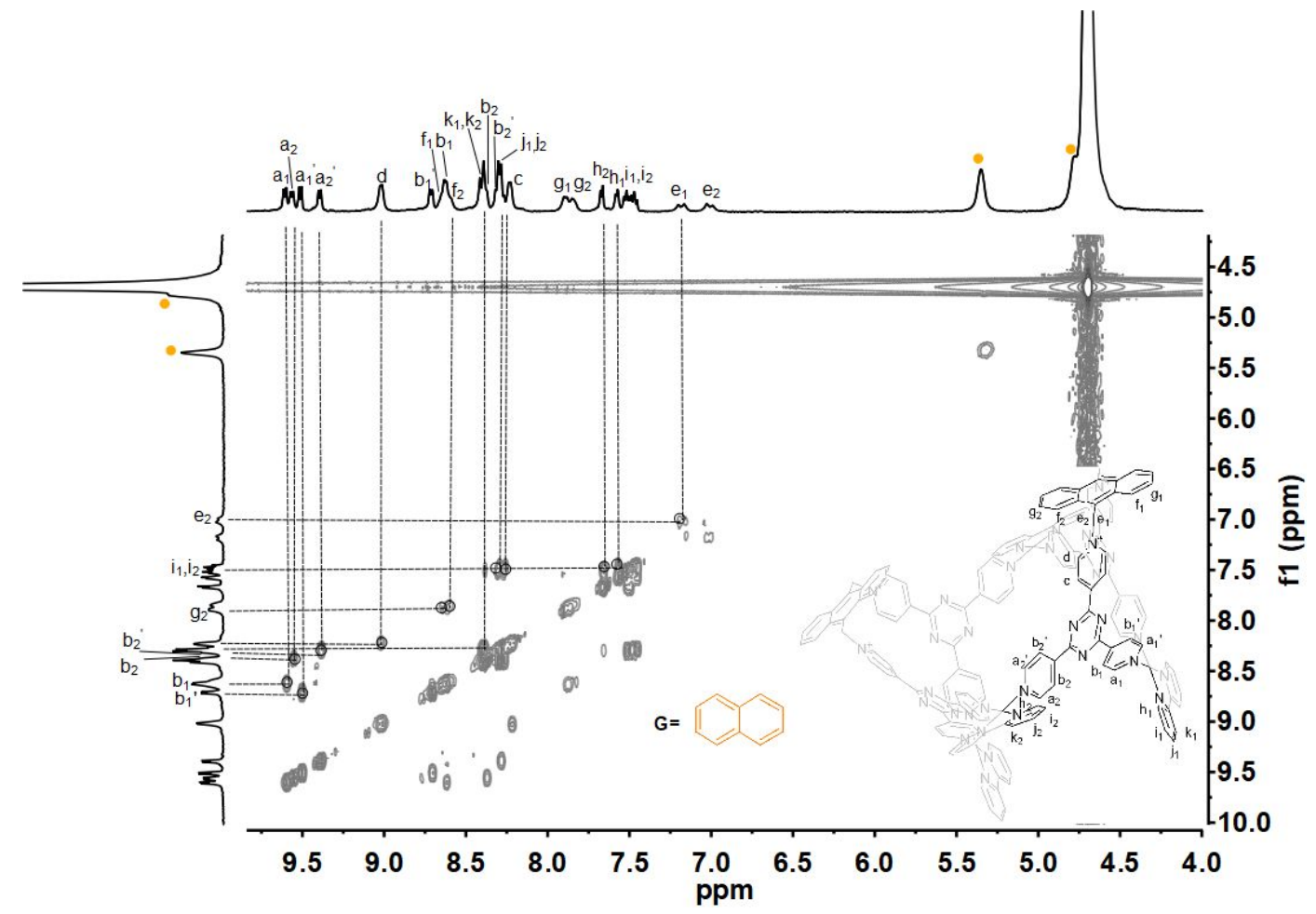

Figure S27. ${ }^{1} \mathrm{H}-{ }^{1} \mathrm{H}$ COSY MNR spectrum $\left(400 \mathrm{MHz}, \mathrm{D}_{2} \mathrm{O}, 298 \mathrm{~K}\right)$ of $\mathbf{N A P}_{3} \subset$ bowl 3 transformed from capsule 2. $(\bullet=$ Guest $)$

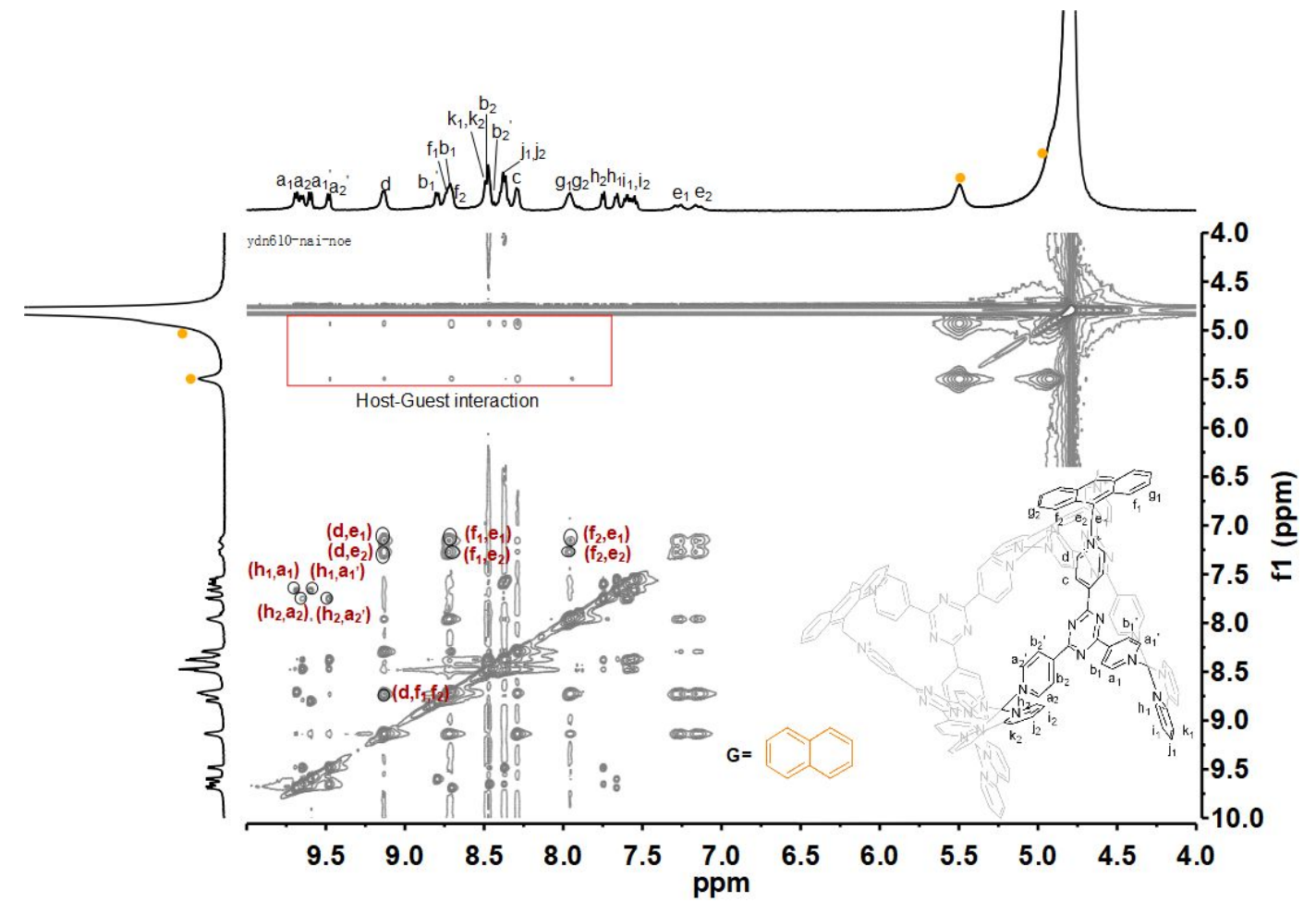

Figure S28. ${ }^{1} \mathrm{H}-{ }^{1} \mathrm{H}$ NOESY spectrum $\left(400 \mathrm{MHz}, \mathrm{D}_{2} \mathrm{O}, 298 \mathrm{~K}\right)$ of $\mathbf{N A P}_{3} \subset$ bowl 3 transformed from capsule 2. $(\bullet=$ Guest $)$ 


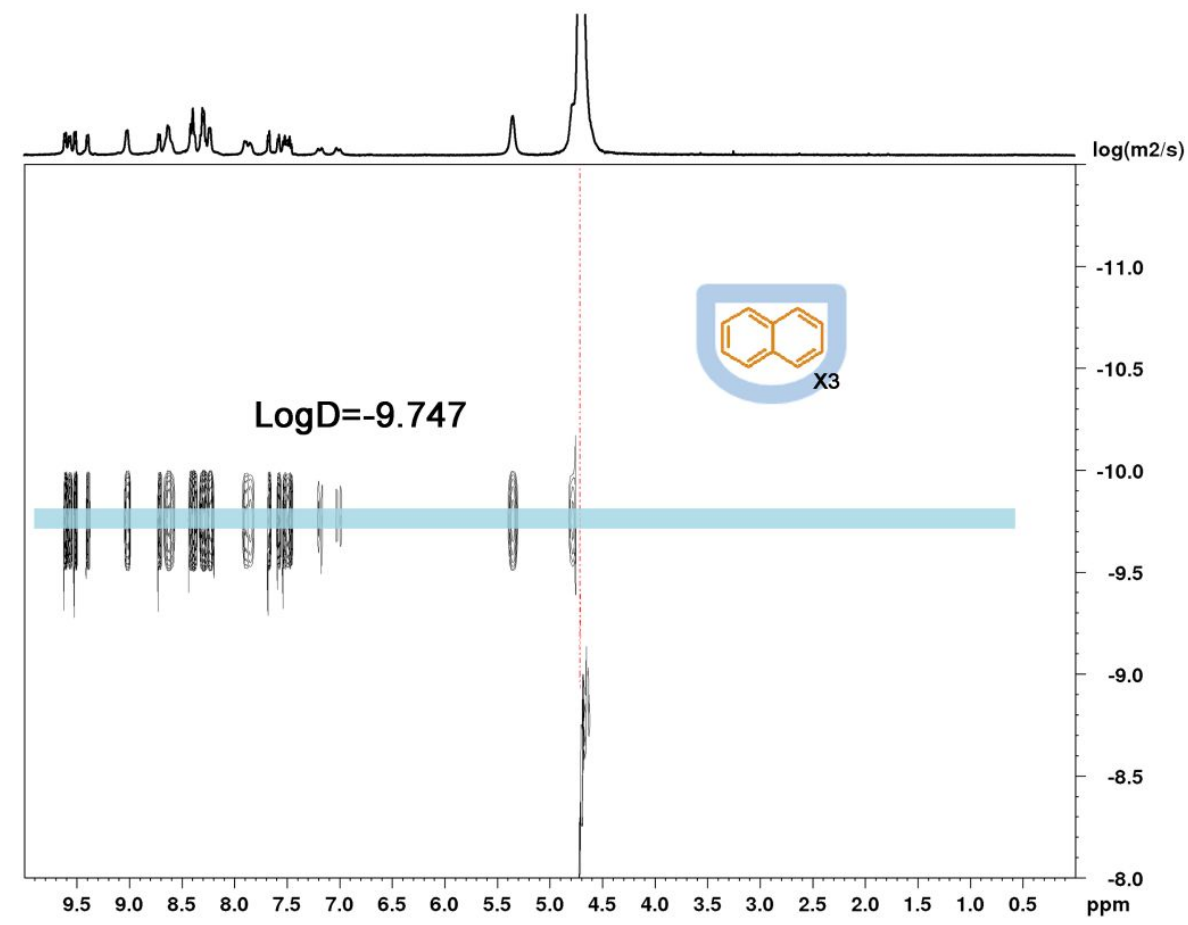

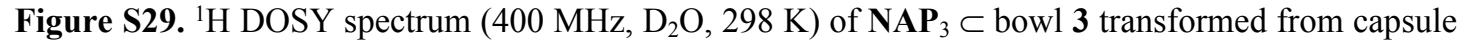
2. $\left(\right.$ Diffusion Constant $=1.79 \mathrm{E}^{-10} \mathrm{~m}^{2} / \mathrm{S}, \mathrm{d}=2.73 \mathrm{~nm}$ )

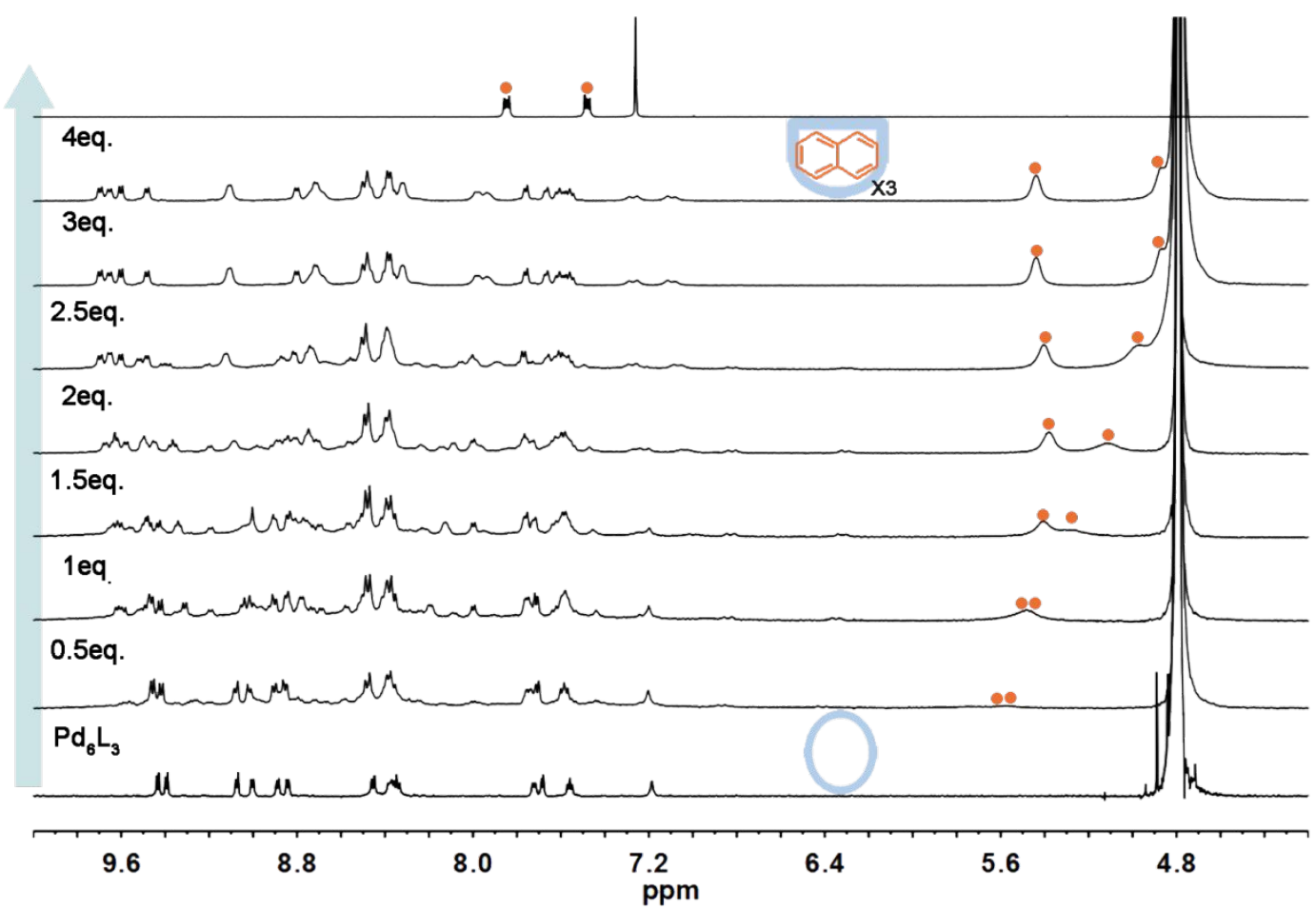

Figure S30. ${ }^{1} \mathrm{H}$ NMR spectra $\left(400 \mathrm{~Hz}, \mathrm{D}_{2} \mathrm{O}, 298 \mathrm{~K}\right)$ of capsule 2 when titrated with NAP, showing fast exchange binding kinetics on the NMR timescale $(\bullet=$ Guest). A 1:3 binding stoichiometry is feasible based on the crystal structure of the $\mathbf{M N S}_{3} \subset$ bowl $\mathbf{3}$ complex (see the crystal data section for details). 
4.5 Formation of inclusion complex (thionaphthene) ${ }_{3} \subset$ bowl 3:

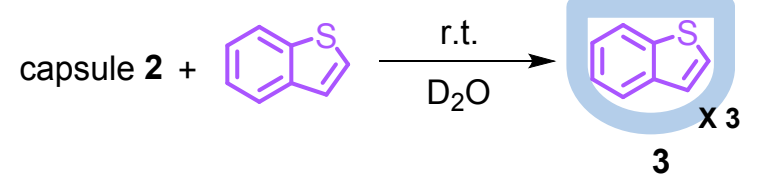

Capsule 2 (5.30 mg, $1.00 \mathrm{mmol}, 1 \mathrm{~mL} \mathrm{D}$ O), thionaphthene (THP, 6 eq., 0.80 mg, liquid), were added to a glass vial and then the mixture was stirred at r.t. for 10 min. The formation of $\mathbf{T} \mathbf{H} \mathbf{P}_{3} \subset \mathbf{3}$ complex was confirmed based on the integration ratio after filtration of the excess THP. This host-guest compound was susceptible to precipitate when adding excess guest, thus thionaphthene was not used for a substrate below for photocatalysis. ${ }^{1} \mathrm{H} \mathrm{NMR}\left(600 \mathrm{MHz}, \mathrm{D}_{2} \mathrm{O}, 298 \mathrm{~K}\right): \delta 9.64(\mathrm{~d}, J=6.0 \mathrm{~Hz}$, $1 \mathrm{H}), 9.61(\mathrm{~d}, J=6.0 \mathrm{~Hz}, 1 \mathrm{H}), 9.56(\mathrm{~d}, J=6.0 \mathrm{~Hz}, 1 \mathrm{H}), 9.44(\mathrm{~d}, J=6.0 \mathrm{~Hz}, 1 \mathrm{H})$, 9.18-9.07 (m, 2H), $8.81(\mathrm{~d}, J=4.0 \mathrm{~Hz}), 8.74-8.68(\mathrm{~m}, 2 \mathrm{H}), 8.71(\mathrm{~m}, 1 \mathrm{H}), 8.60-8.53$ $(\mathrm{m}, 1 \mathrm{H}), 8.48-8.45(\mathrm{~m}, 3 \mathrm{H}), 8.43-8.39(\mathrm{~m}, 3 \mathrm{H}), 8.38-8.34(\mathrm{~m}, 2 \mathrm{H}), 8.00-7.91(\mathrm{~m}, 1 \mathrm{H})$, 7.75-7.70 (m, 2H), $7.67(\mathrm{~d}, J=5.4 \mathrm{~Hz}, 1 \mathrm{H}), 7.58(\mathrm{t}, J=6.6 \mathrm{~Hz}, 1 \mathrm{H}), 7.54(\mathrm{t}, J=6.6 \mathrm{hz}$, $1 \mathrm{H}), 7.25(\mathrm{~d}, J=12 \mathrm{~Hz}, 1 \mathrm{H}), 7.03(\mathrm{~d}, J=12 \mathrm{~Hz}, 1 \mathrm{H}), 6.17-4.99(\mathrm{~m}, 4 \mathrm{H})$.

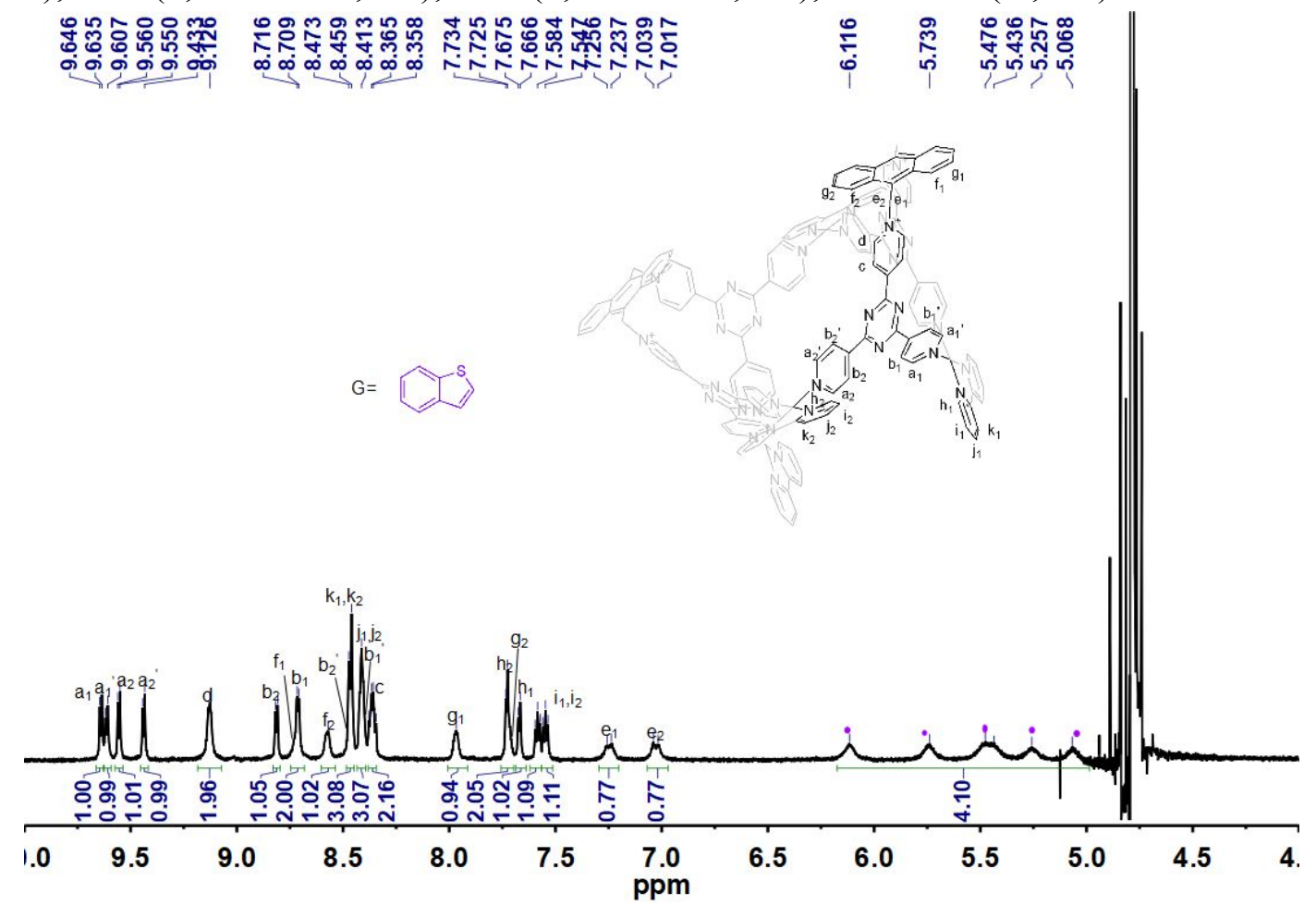

Figure S31. ${ }^{1} \mathrm{H}$ NMR spectrum $\left(600 \mathrm{MHz}, \mathrm{D}_{2} \mathrm{O}, 298 \mathrm{~K}\right)$ of $\mathbf{T H P}_{3} \subset$ bowl 3 transformed from capsule 2. $(\bullet=$ Guest $)$ 


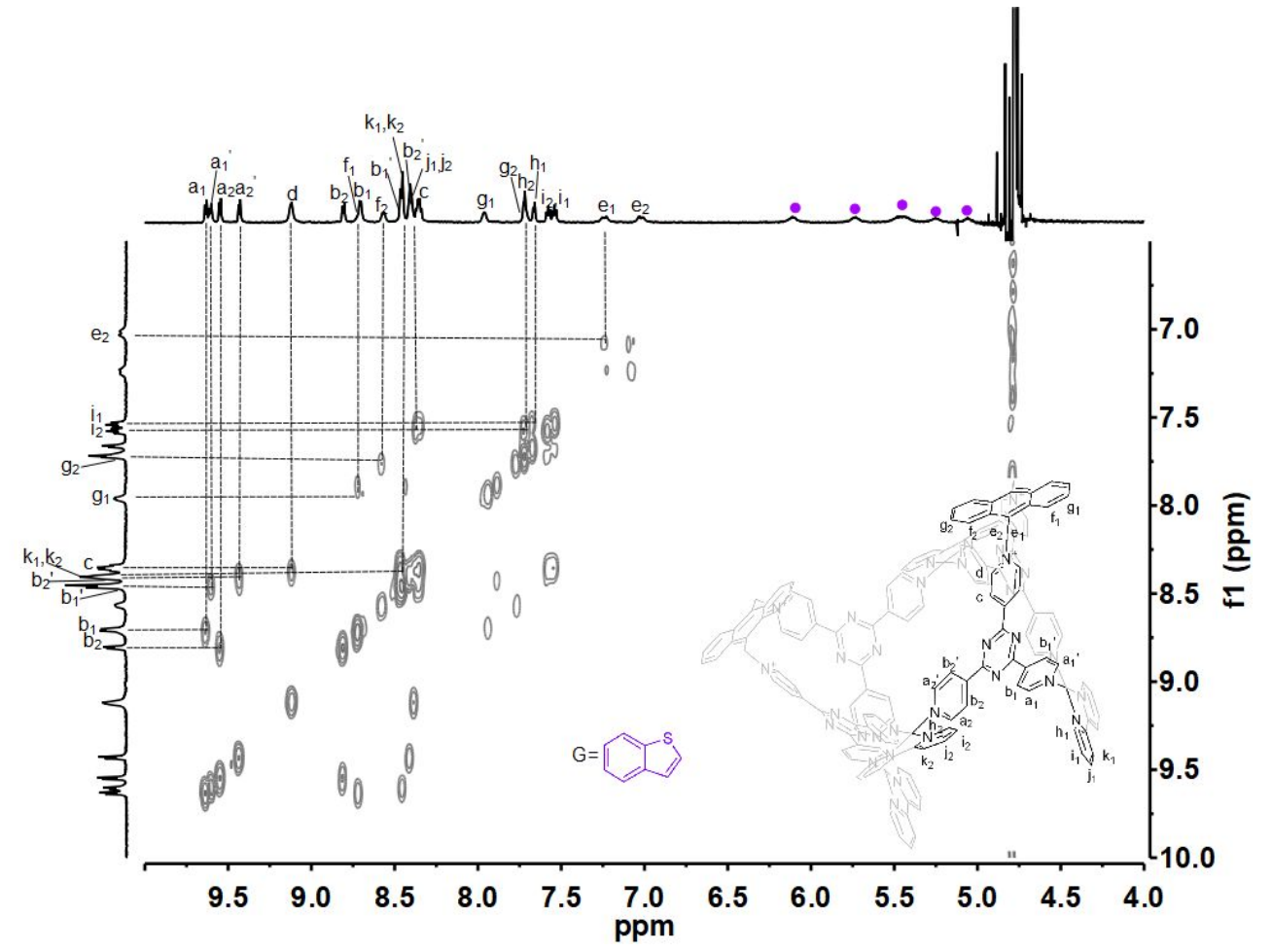

Figure S32. ${ }^{1} \mathrm{H}-{ }^{1} \mathrm{H}$ COSY spectrum $\left(600 \mathrm{MHz}, \mathrm{D}_{2} \mathrm{O}, 298 \mathrm{~K}\right)$ of $\mathbf{T H P}_{3} \subset$ bowl 3 transformed from capsule 2. $(\bullet=$ Guest $)$

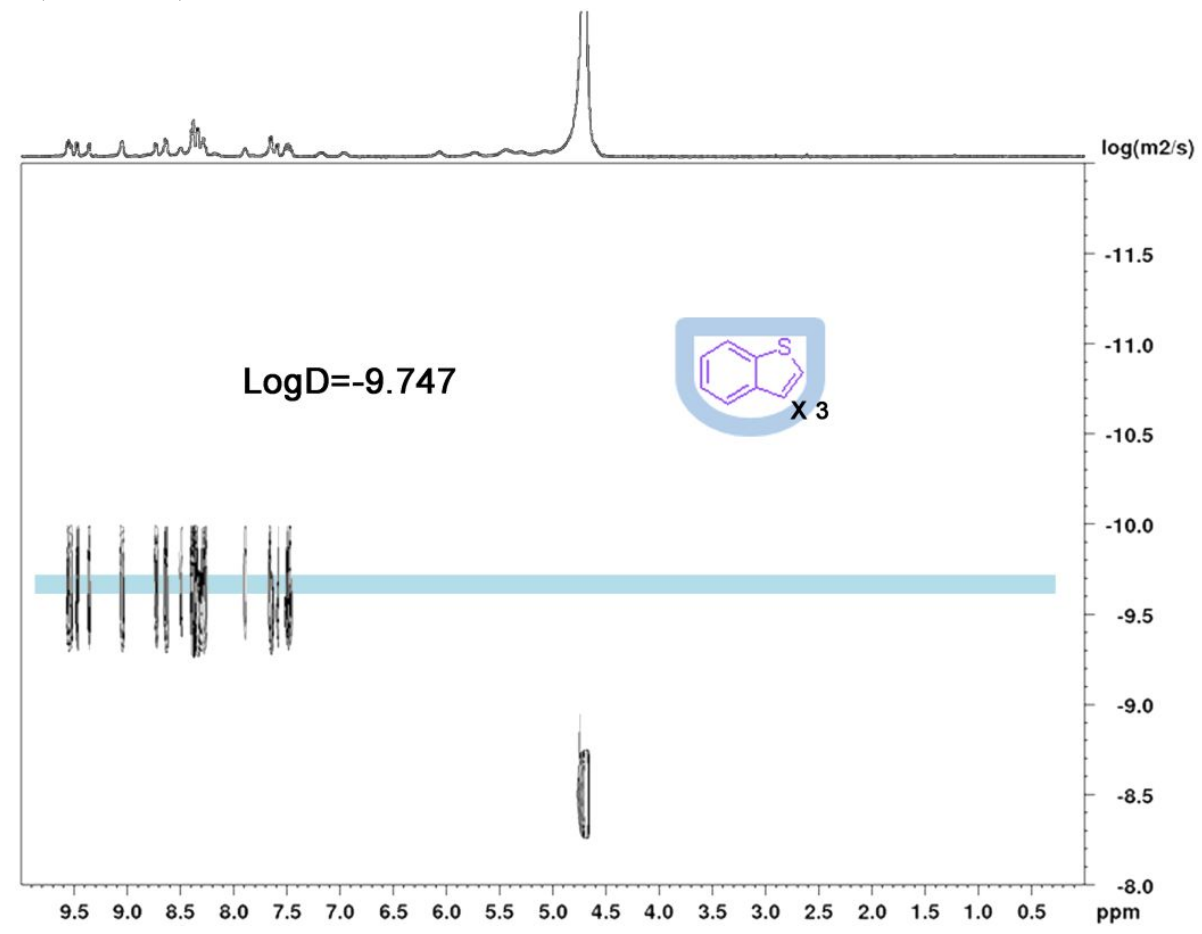

Figure S33. ${ }^{1} \mathrm{H}$ DOSY spectrum $\left(400 \mathrm{MHz}, \mathrm{D}_{2} \mathrm{O}, 298 \mathrm{~K}\right)$ of $\mathbf{T H P}_{3} \subset$ bowl 3 transformed from capsule 2. (Diffusion Constant $=1.79 \mathrm{E}^{-10} \mathrm{~m}^{2} / \mathrm{S}, \mathrm{d}=2.73 \mathrm{~nm}$ ) 


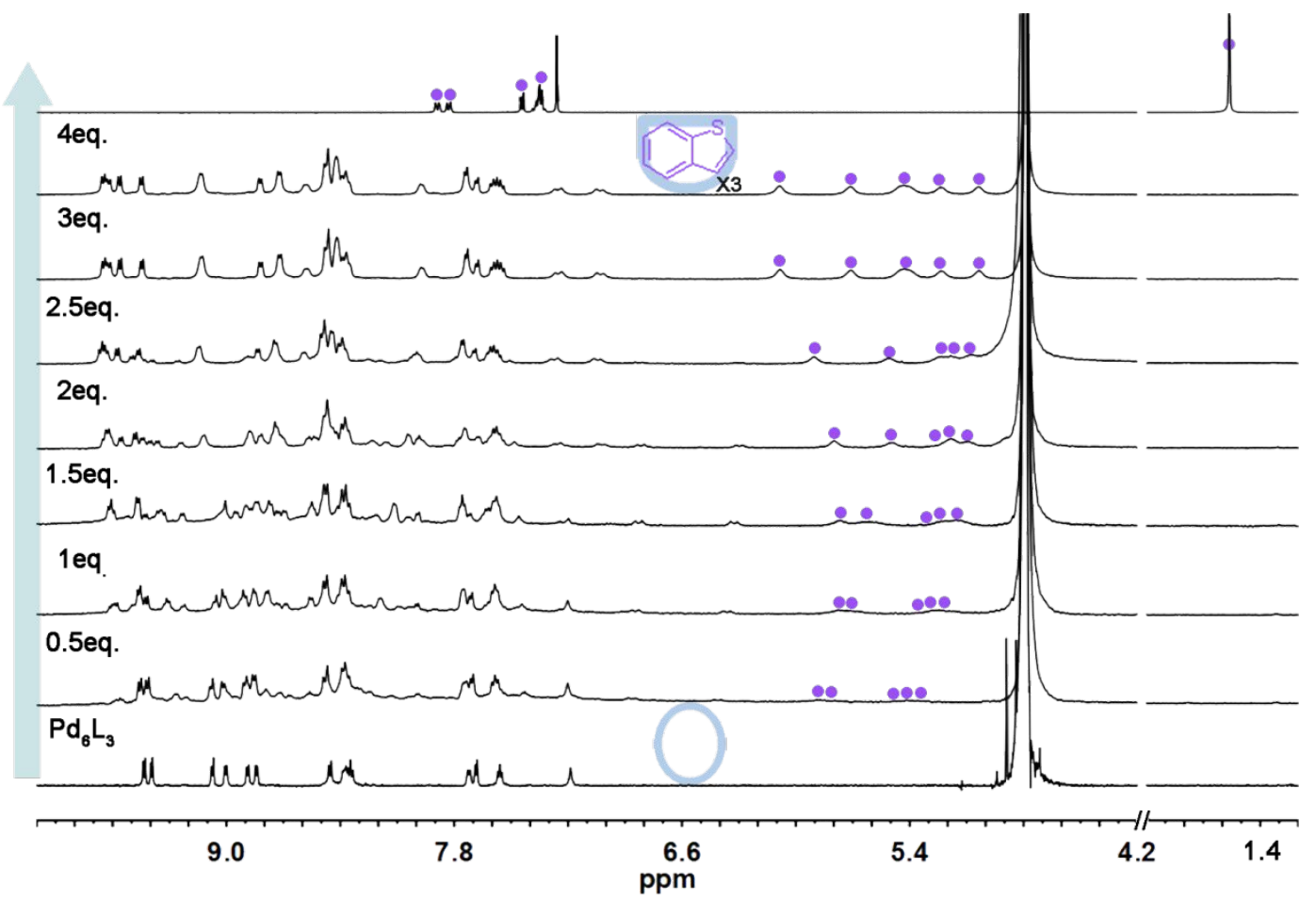

Figure S34. ${ }^{1} \mathrm{H}$ NMR spectra $\left(400 \mathrm{~Hz}, \mathrm{D}_{2} \mathrm{O}, 298 \mathrm{~K}\right)$ of capsule 2 when titrated with THP, showing fast exchange binding kinetics on the NMR timescale $(\bullet=$ Guest $)$. A 1:3 binding stoichiometry is feasible based on the crystal structure of the $\mathbf{M N S}_{3} \subset$ bowl $\mathbf{3}$ complex (see the crystal data section for details).

\subsection{In-situ guest-template self-assembly of $L^{2}, P d$, and naphthalene}

$$
\mathrm{L}^{2}+(\mathrm{bpy}) \mathrm{Pd}\left(\mathrm{NO}_{3}\right)_{2}+
$$

Ligand $\mathrm{L}^{2}(3.00 \mathrm{mg}, 3.00 \mathrm{mmol})$, (bpy) $\mathrm{Pd}\left(\mathrm{NO}_{3}\right)_{2}(2.30 \mathrm{mg}, 6.00 \mathrm{mmol})$, naphthalene (NAP, 10 eq., $1.28 \mathrm{mg}$, a template), $\mathrm{D}_{2} \mathrm{O}(0.60 \mathrm{~mL})$ were added to a glass vial and then the mixture was stirred at $80^{\circ} \mathrm{C}$ until furnishing a clear solution. 


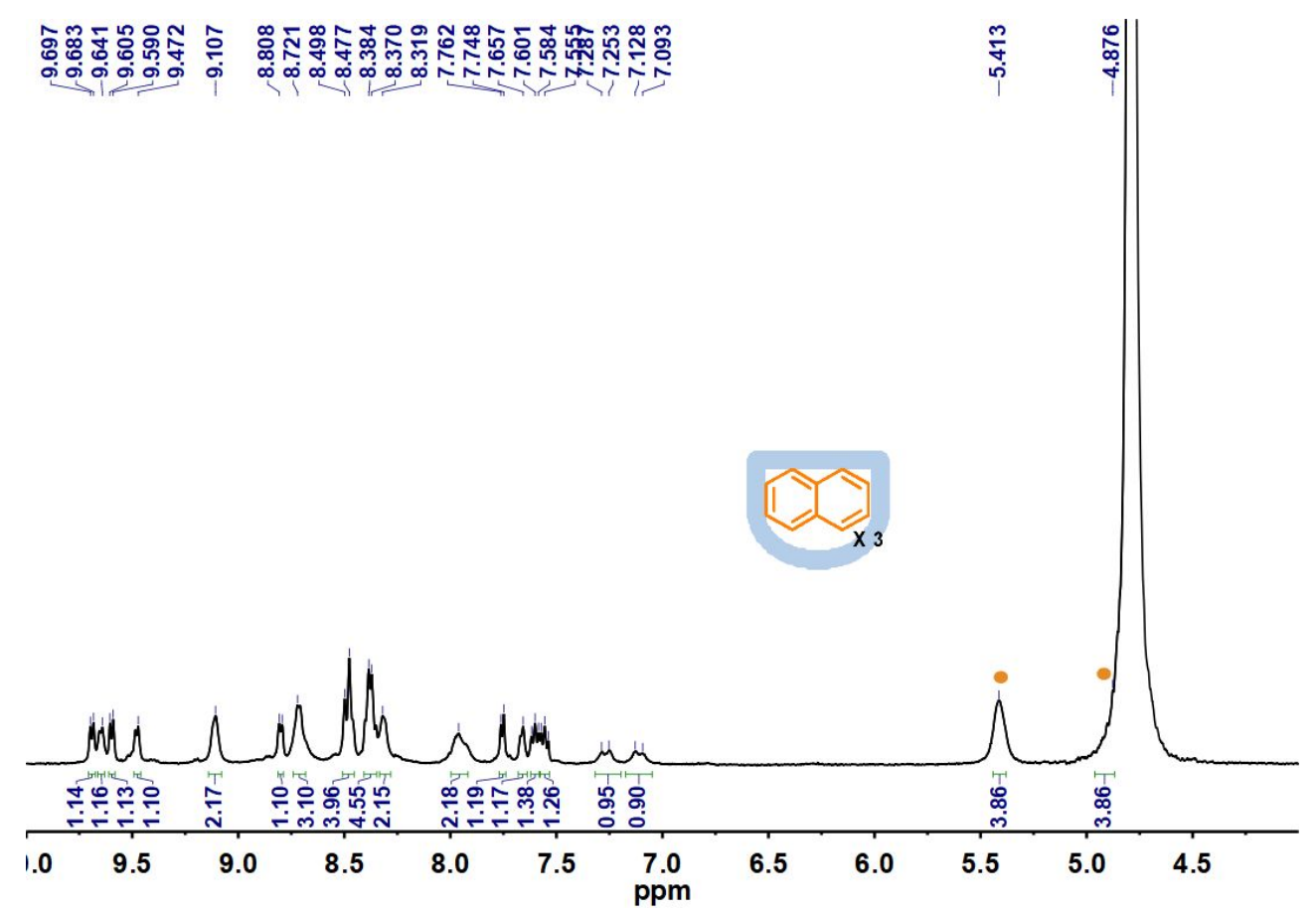

Figure S35. ${ }^{1} \mathrm{H}$ NMR spectrum (400 MHz, $\left.\mathrm{D}_{2} \mathrm{O}, 298 \mathrm{~K}\right)$ of bowl 3 hosting naphthalene by in-situ guest-template self-assembly. $(\bullet=$ Guest $)$

\subsection{The conversion from capsule 2 to bowl 3 over time:}

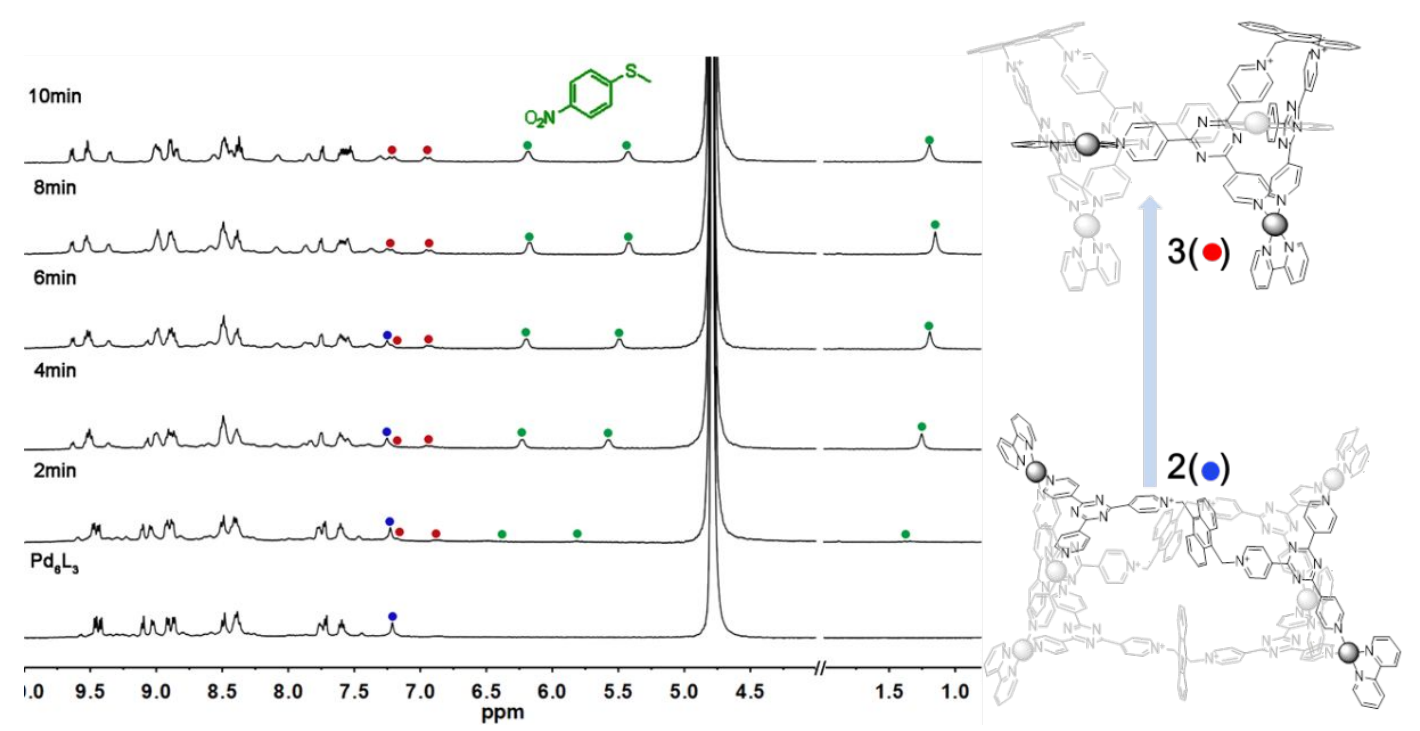

Figure S36. Time-dependent ${ }^{1} \mathrm{H}$ NMR spectra $\left(400 \mathrm{~Hz}, \mathrm{D}_{2} \mathrm{O}, 298 \mathrm{~K}\right)$ for capsule 2 to bowl 3 conversions. The addition of MNS to a solution of $\mathbf{2}$ leads to the quantitative transformation of $\mathbf{M N S}_{3}$ $\subset$ 3. The characteristic methylene signals $\left(\mathrm{H}_{\mathrm{e}}\right)$ on capsule $\mathbf{2}$ and bowl $\mathbf{3}$ were labeled with $\bullet$ and $\bullet$ respectively. $(\bullet=$ Guest $)$

\subsection{The reversible inter-conversion between capsule 2 and bowl 3:}

The reversible transformation from bowl $\mathbf{3}$ to capsule $\mathbf{2}$ was realized by removal 
of guest molecules with solvent extraction or adding an excess amount of ADC guest.

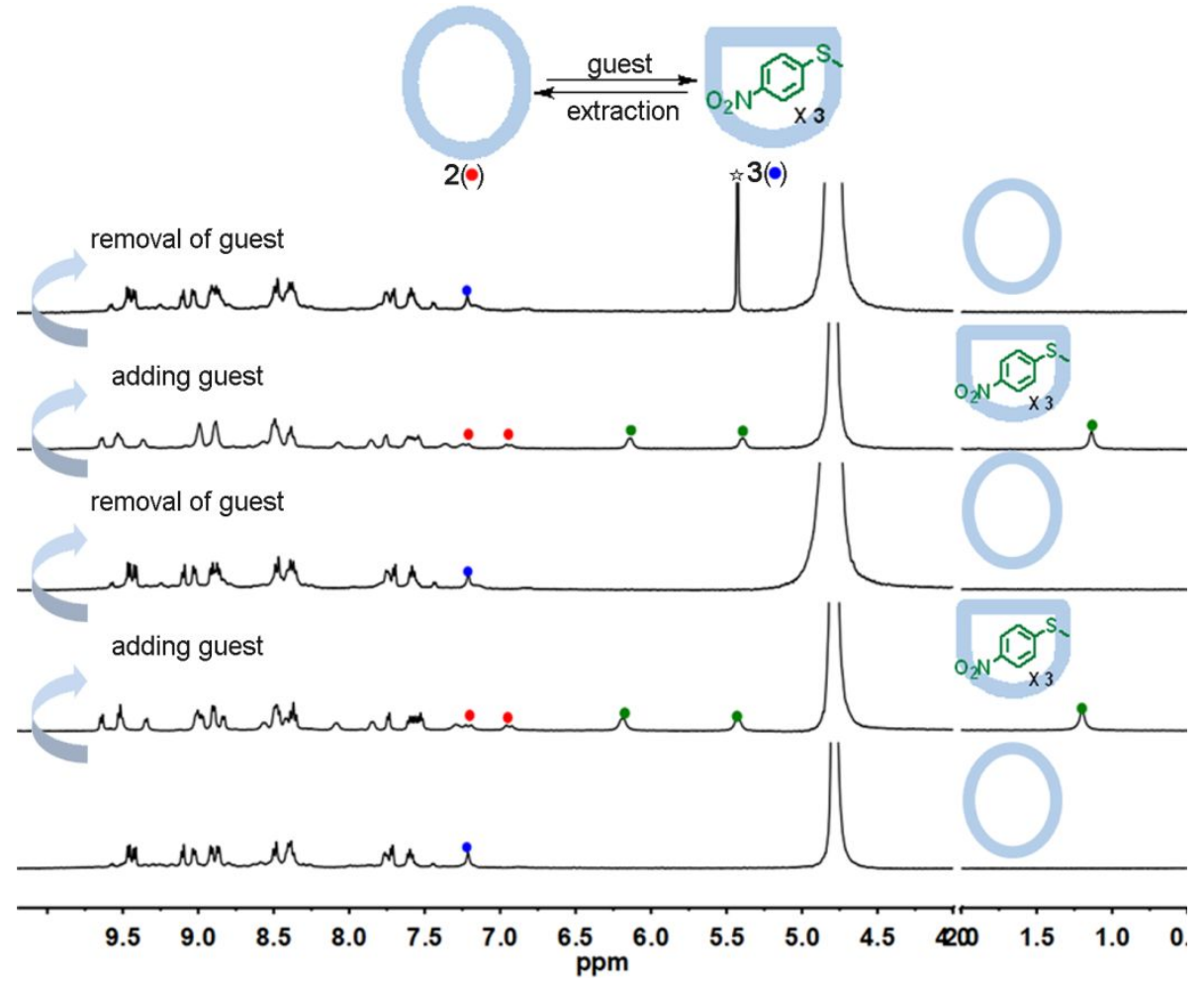

Figure S37. ${ }^{1} \mathrm{H}$ NMR spectra ( $400 \mathrm{MHz}, \mathrm{D}_{2} \mathrm{O}, 298 \mathrm{~K}$ ) demonstrating the reversible inter-conversion between capsule $2\left(\mathrm{Pd}_{6} \mathrm{~L}_{3}^{2}\right)$ and bowl $3\left(\mathrm{Pd}_{4} \mathrm{~L}_{2}^{2}\right)$ by addition or removal guest molecules using $\mathrm{CH}_{2} \mathrm{Cl}_{2}$ extraction. (The characteristic methylene signals $\left(\mathrm{H}_{\mathrm{e}}\right)$ on capsule $\mathbf{2}$ and bowl $\mathbf{3}$ were labeled with $\bullet$ and - respectively. ( $\bullet=$ Guest, Residual solvent signals were labeled as a star.)

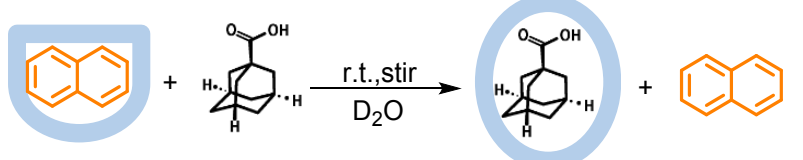

After adding ADC

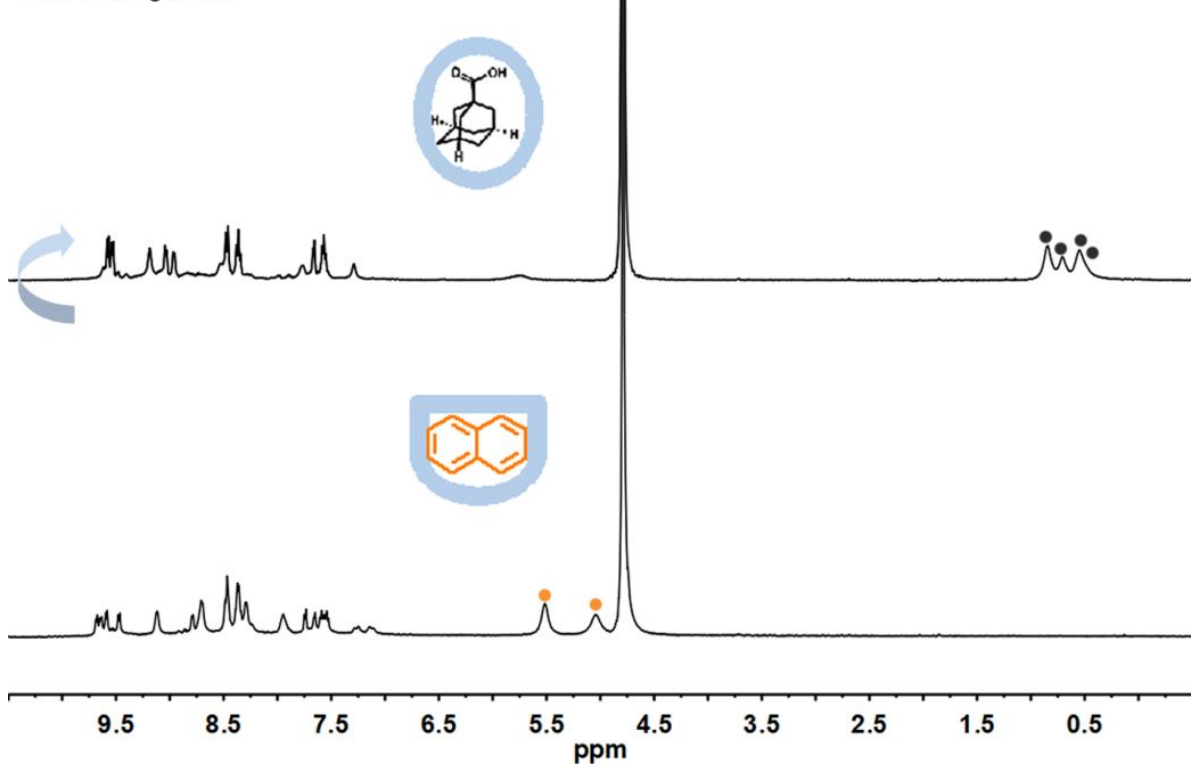


Figure S38. ${ }^{1} \mathrm{H}$ NMR spectra $\left(400 \mathrm{MHz}, \mathrm{D}_{2} \mathrm{O}, 298 \mathrm{~K}\right)$ of $\mathbf{N A P}_{3} \subset$ bowl 3 (below) and after adding 10 eq. $\mathbf{A D C}$ to $\mathbf{N A P} \mathbf{P}_{3} \subset$ bowl $\mathbf{3}$ (top).

\section{The host-guest behavior of cage 1:}

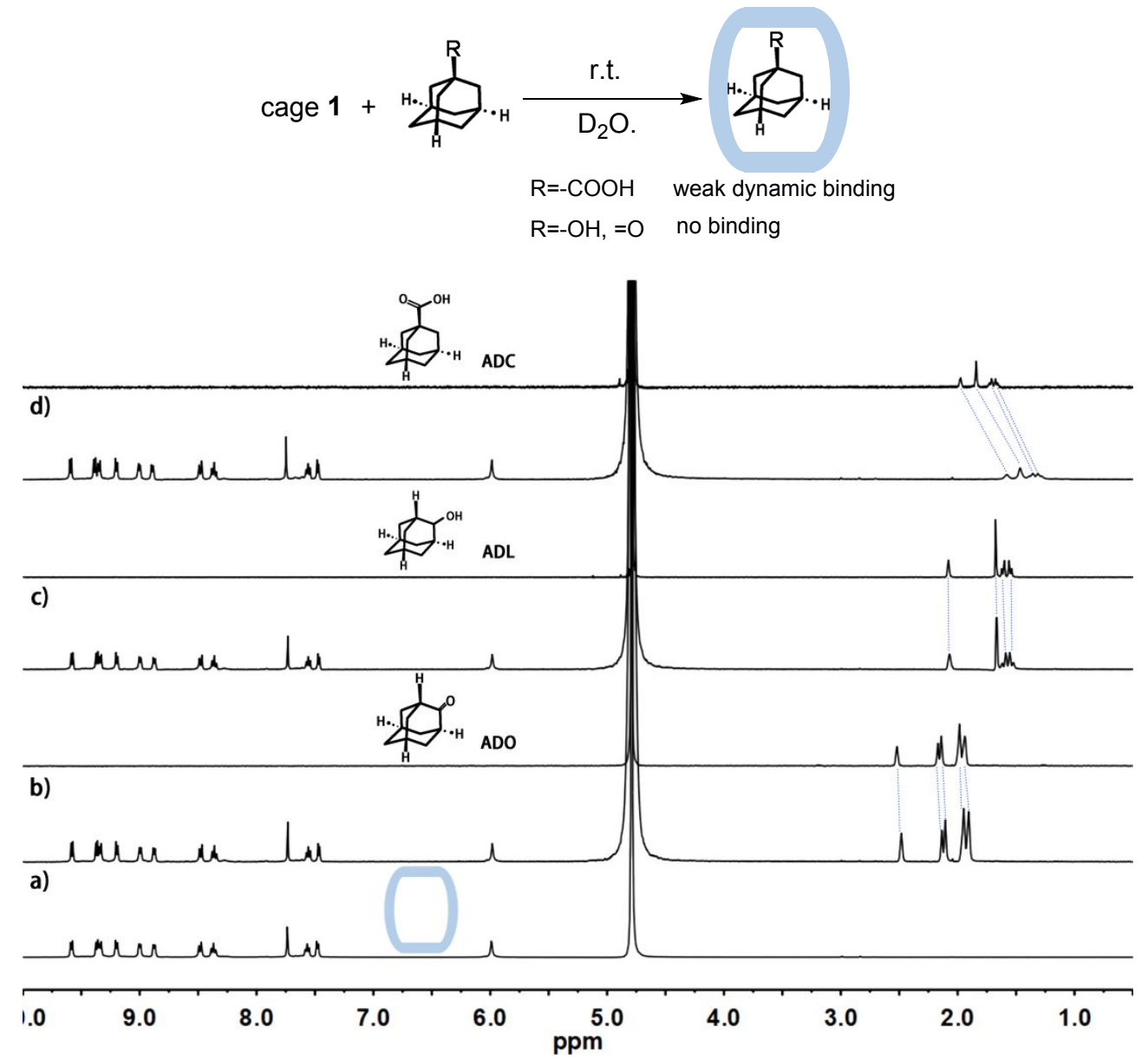

Figure S39. ${ }^{1} \mathrm{H}$ NMR spectra $\left(400 \mathrm{MHz}, \mathrm{D}_{2} \mathrm{O}, 298 \mathrm{~K}\right)$ of cage $\mathbf{1}$ and cage $\mathbf{1}$ after adding excess adamantane derivatives (ADC, ADL, ADO) as guests. 


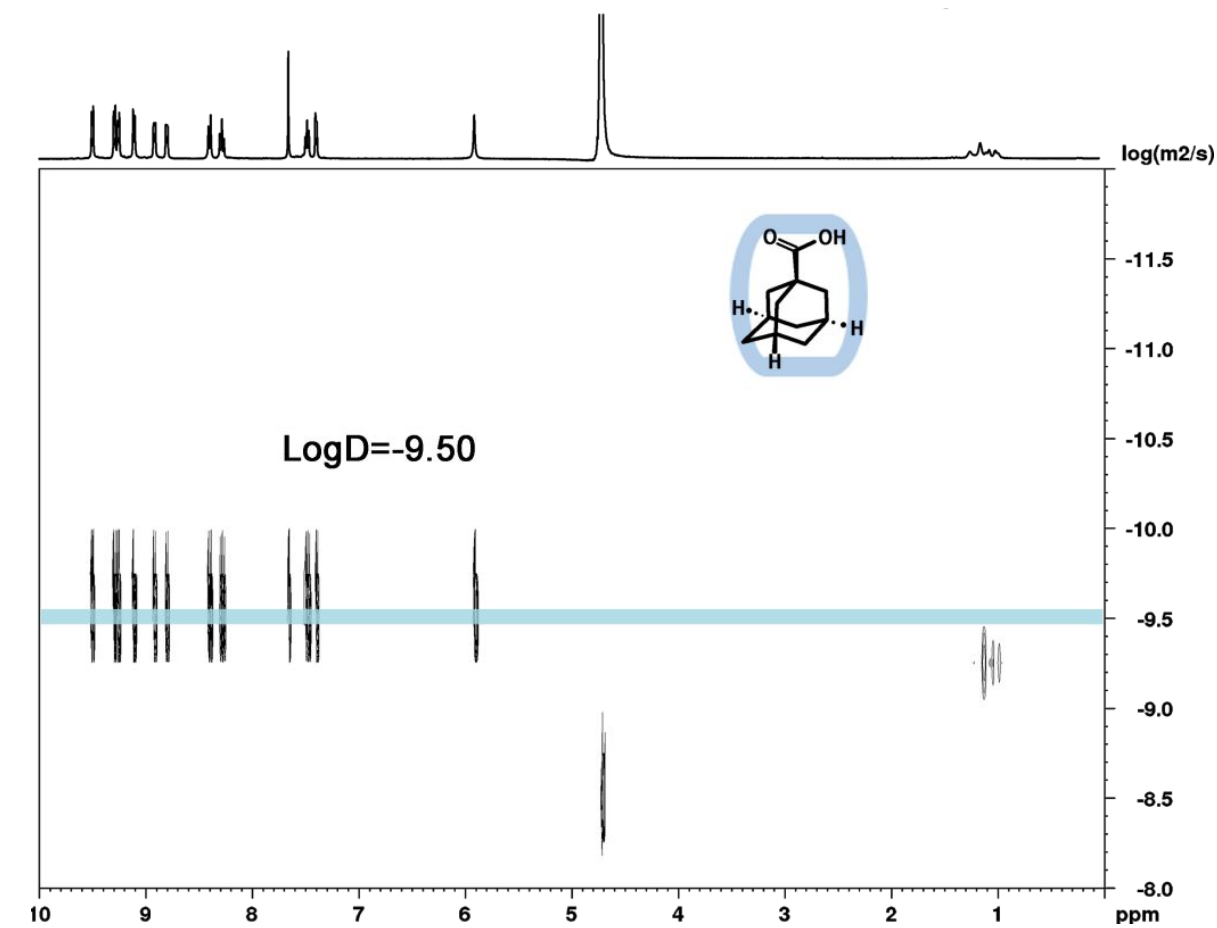

Figure S40. DOSY spectrum (400 MHz, $\mathrm{D}_{2} \mathrm{O}, 298 \mathrm{~K}$ ) of cage 1 after adding ADC. (Diffusion Constant $\left.=2.63 \mathrm{E}^{-10} \mathrm{~m}^{2} / \mathrm{S}, \mathrm{d}=1.86 \mathrm{~nm}\right)$

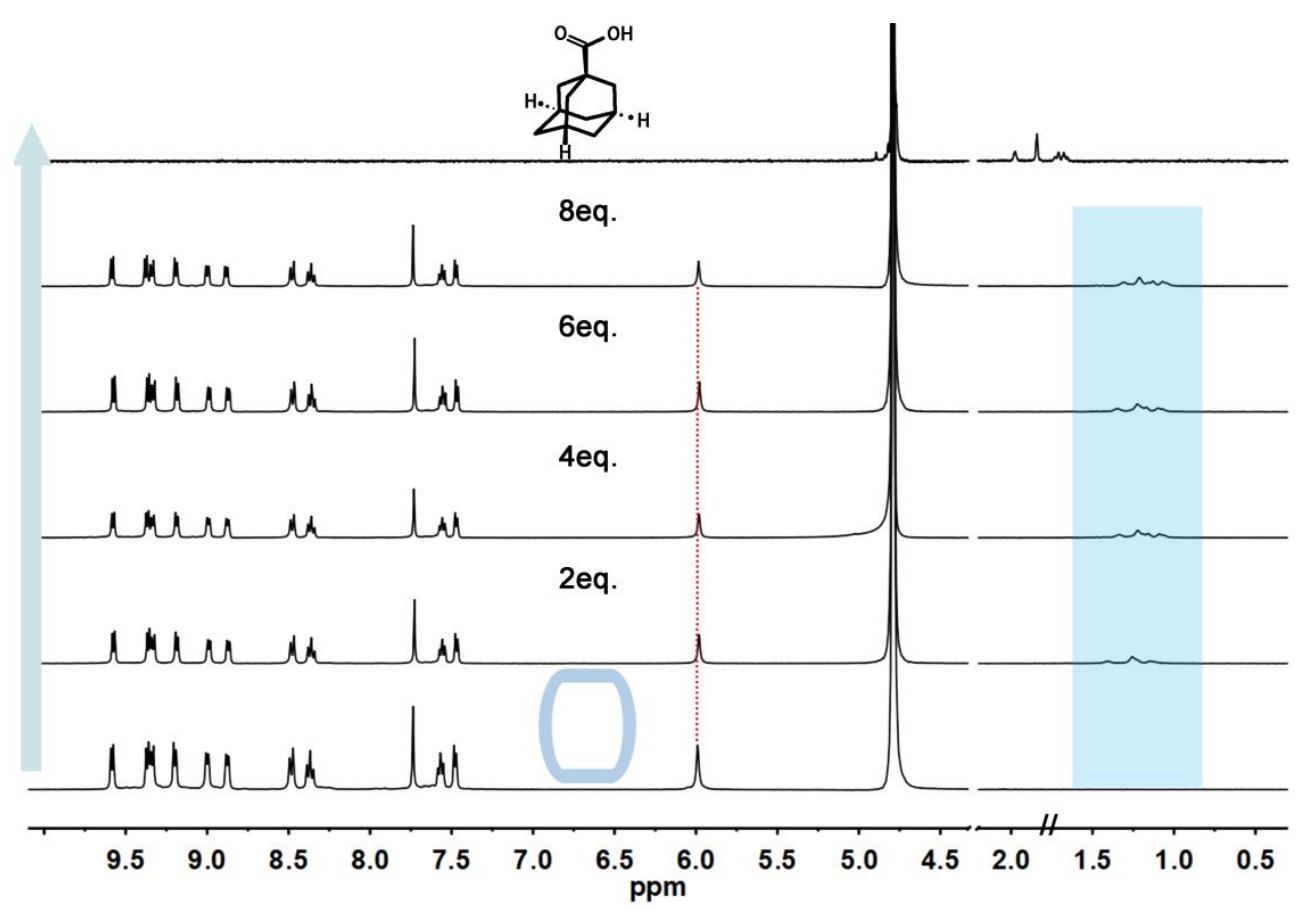

Figure S41. ${ }^{1} \mathrm{H}$ NMR spectra ( $400 \mathrm{MHz}, \mathrm{D}_{2} \mathrm{O}, 298 \mathrm{~K}$ ) of cage 1 when titrated with ADC (0-8 eq.), showing no evident shift on host, indicative of fast exchange on the NMR timescale. 


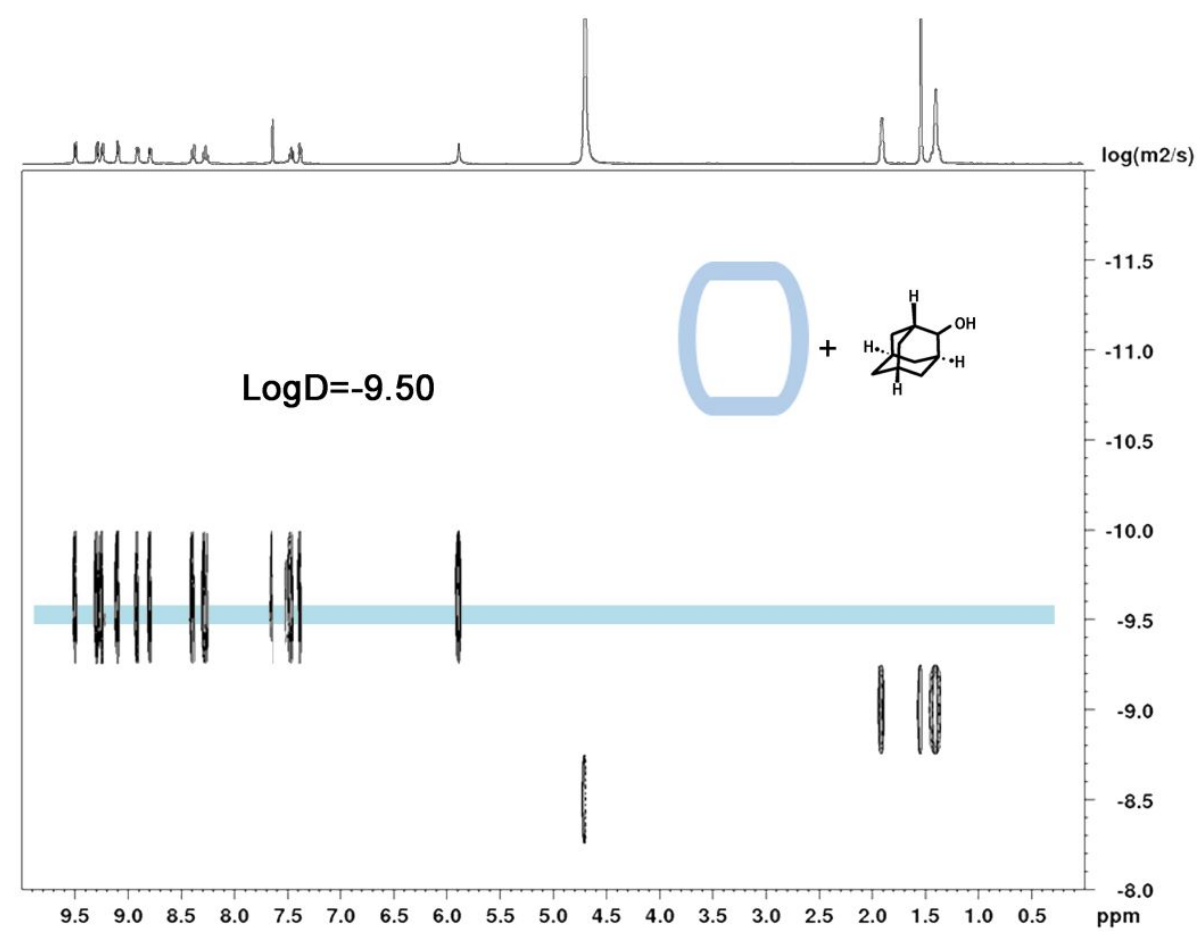

Figure S42. DOSY spectrum ( $400 \mathrm{MHz}, \mathrm{D}_{2} \mathrm{O}, 298 \mathrm{~K}$ ) of cage 1 after adding ADL. (Diffusion Constant $\left.=2.63 \mathrm{E}^{-10} \mathrm{~m}^{2} / \mathrm{S}, \mathrm{d}=1.86 \mathrm{~nm}\right)$

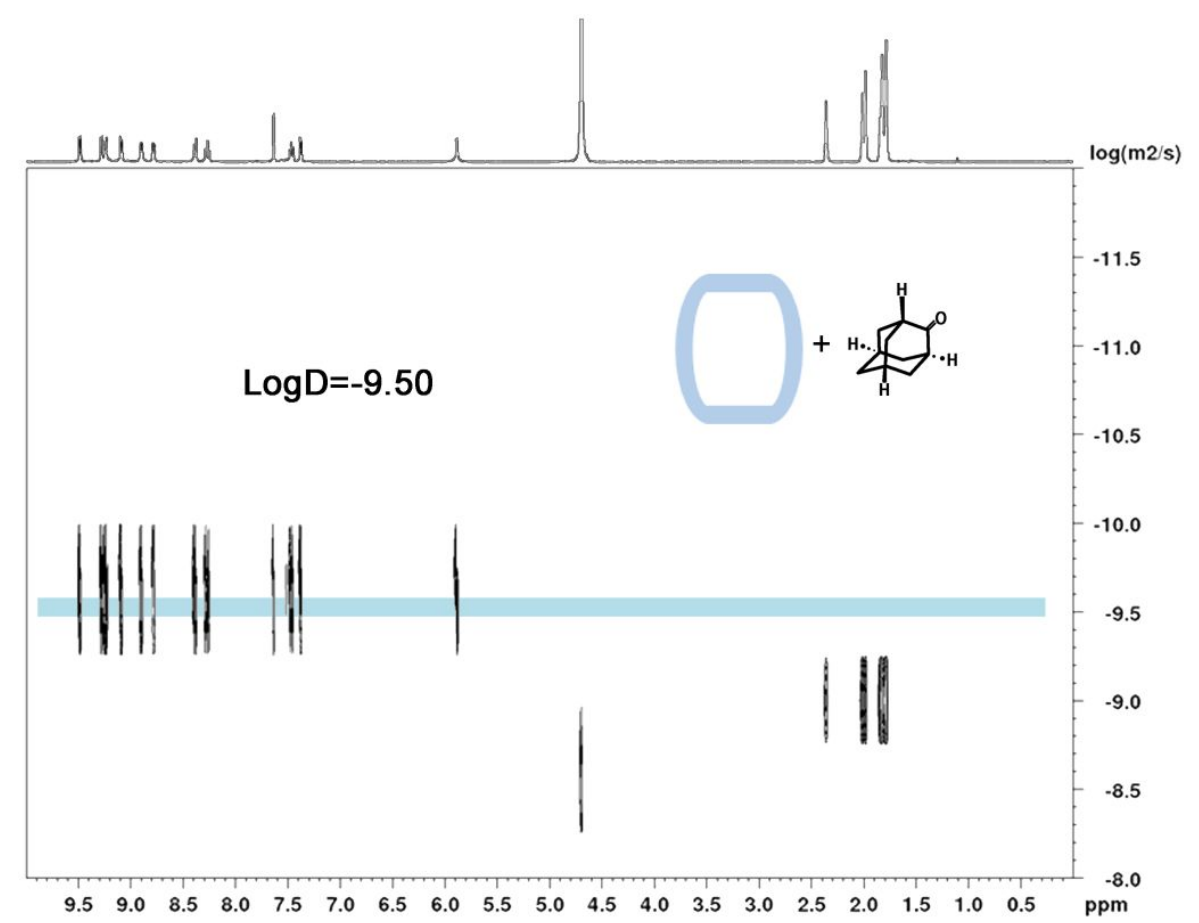

Figure S43. DOSY spectrum ( $400 \mathrm{MHz}, \mathrm{D}_{2} \mathrm{O}, 298 \mathrm{~K}$ ) of cage $\mathbf{1}$ after adding ADO. (Diffusion Constant $\left.=2.63 \mathrm{E}^{-10} \mathrm{~m}^{2} / \mathrm{S}, \mathrm{d}=1.86 \mathrm{~nm}\right)$ 


$$
\text { cage } 1+\underset{\mathrm{R}}{\mathrm{r} \text { r.t. }} \mathrm{D}_{2} \mathrm{O}
$$

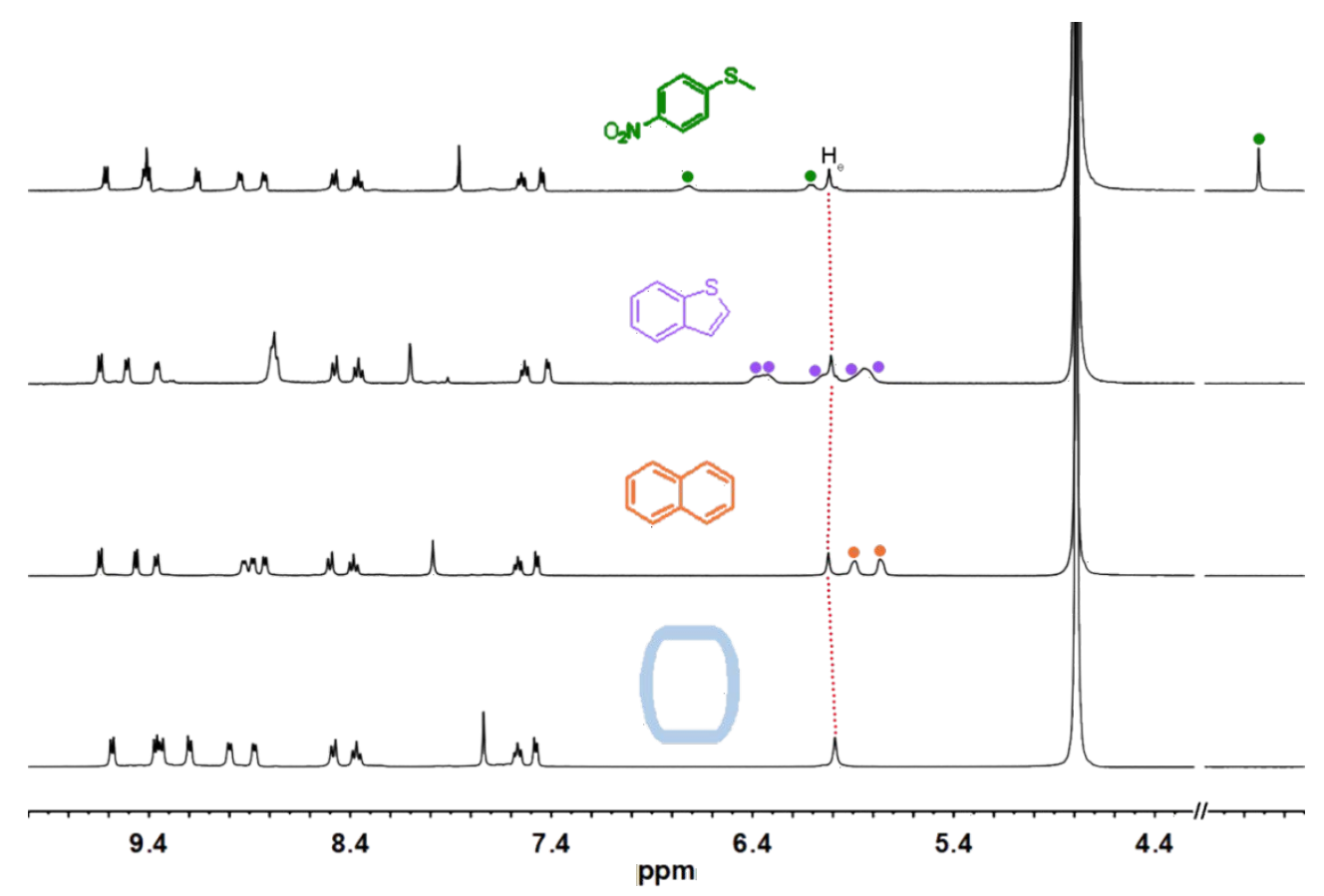

Figure S44. ${ }^{1} \mathrm{H}$ NMR spectra $\left(400 \mathrm{MHz}, \mathrm{D}_{2} \mathrm{O}, 298 \mathrm{~K}\right)$ of inclusion complex after cage 1 adding planar aromatic molecules. Guest signals are labeled with circles in a different color.

\section{Photophysical and redox properties of capsule 2:}

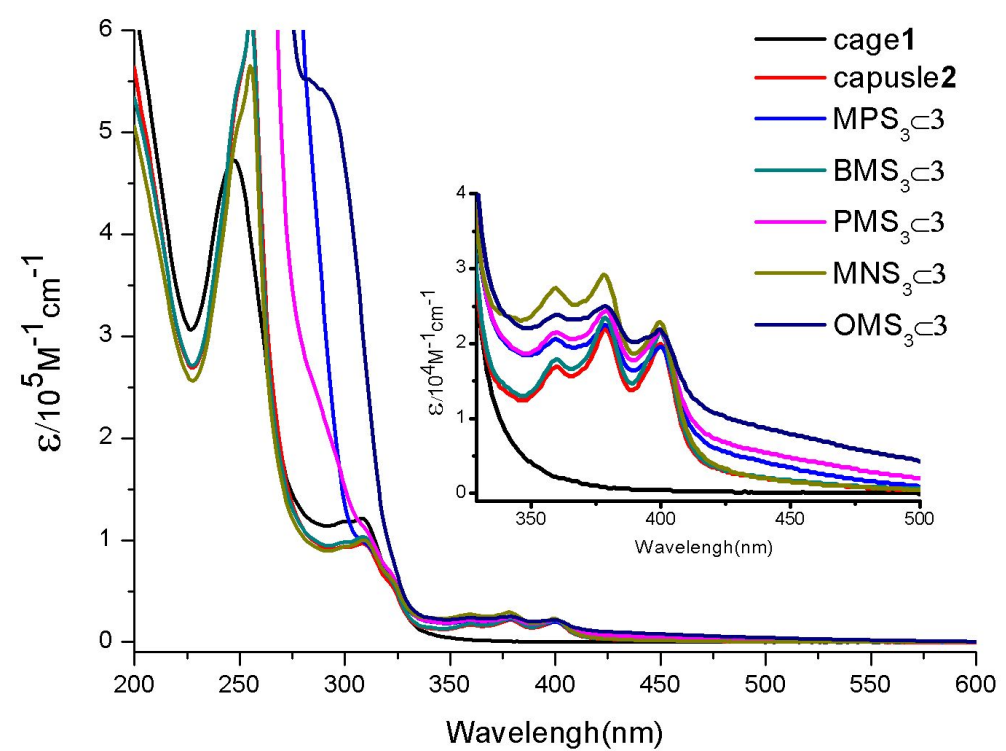

Figure S45. UV/Vis spectra of cage 1, capsule 2, and corresponding inclusion complex in $\mathrm{H}_{2} \mathrm{O}$. (275 $\mathrm{nm}-325 \mathrm{~nm}$ absorption bands belong to excess water-soluble guests) 

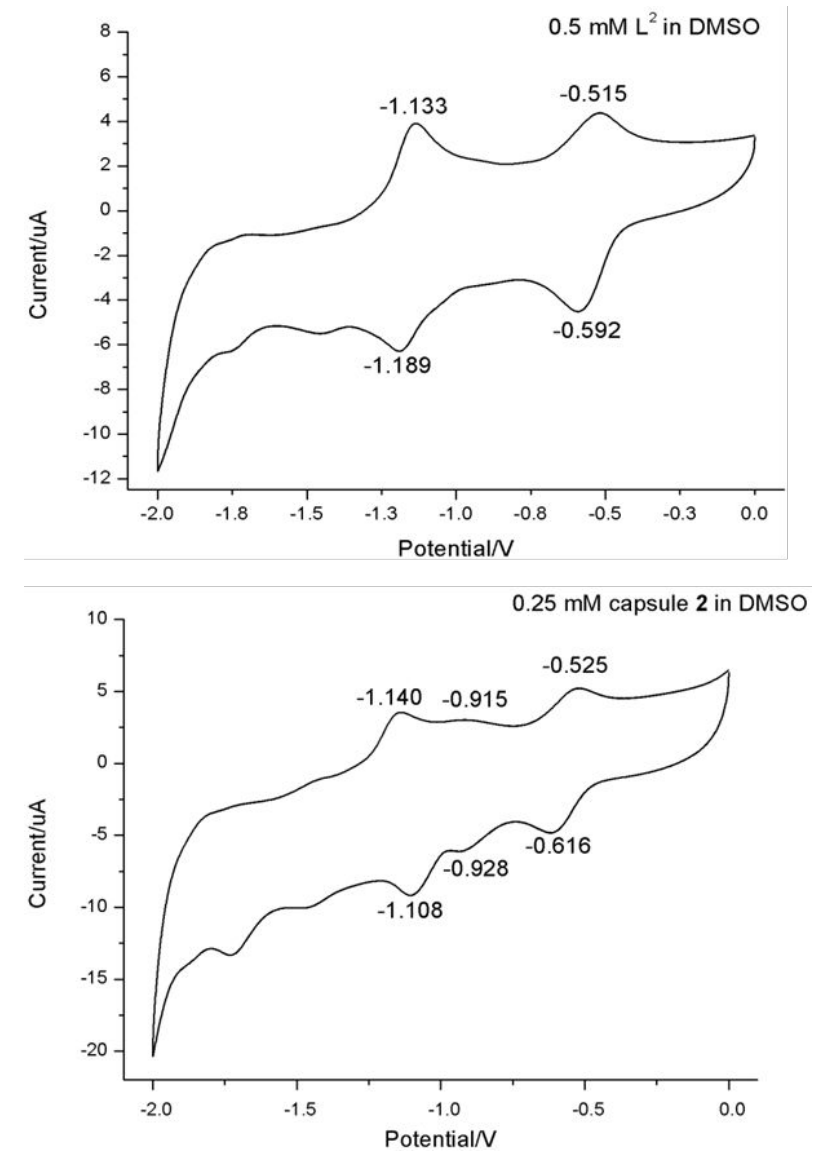

Figure S46. Cyclic voltammograms of ligand $\mathrm{L}^{2}(0.5 \mathrm{mM}$, top) and capsule $2(0.25 \mathrm{mM}$, below) in DMSO at a $0.1 \mathrm{~V} / \mathrm{s}$ scan rate. $\mathrm{Bu}_{4} \mathrm{NPF}_{6}(0.1 \mathrm{M})$ was used as a supporting electrolyte.

\section{Procedure for photocatalysis experiment:}

The photocatalytic reactivity of capsule 2 was tested with methyl(phenyl)sulfane (MPS), (4-bromophenyl)(methyl)sulfane (BMS), ( $p$-tolyl)methylsulfane (PMS), methyl(4-nitrophenyl)sulfane (MNS), 4-methoxyphenyl-methyl-sulfane (OMS) as substrates candidates. Generally, sulfides (10-20 equiv.) were added into the $\mathrm{D}_{2} \mathrm{O}$ solution $(1 \mathrm{~mL})$ containing catalysts $(0.001 \mathrm{mmol}, 1.0$ equiv.). Due to conversion from capsule 2 to bowl $\mathbf{3}$ is very fast compared to the entire photoreaction process, this mixture solution was directly treated under blue LEDs with a magnetic stirring bar and air as an oxidant at r.t. The reaction solution was monitored by thin layer chromatography method. After reaction, the products were extracted with $\mathrm{CHCl}_{3}$ or $\mathrm{CH}_{2} \mathrm{Cl}_{2}(3 \times 5 \mathrm{~mL})$, which were dried under reduce pressure and subjected to ${ }^{1} \mathrm{H}$ NMR without further purification. The conversion and selectivity of sulfoxidation reactions are determined using 1,3,5-trimethoxybenzene as the internal standard $(0.005 \mathrm{mmol})$. Typical peaks assignable to capsule 2 were recovered soon after mild heating of the residual aqueous solution because of host precipitation during extraction process. Control experiments were carried out in the absence of catalysis, light, or $\mathrm{N}_{2}$ atmosphere. 


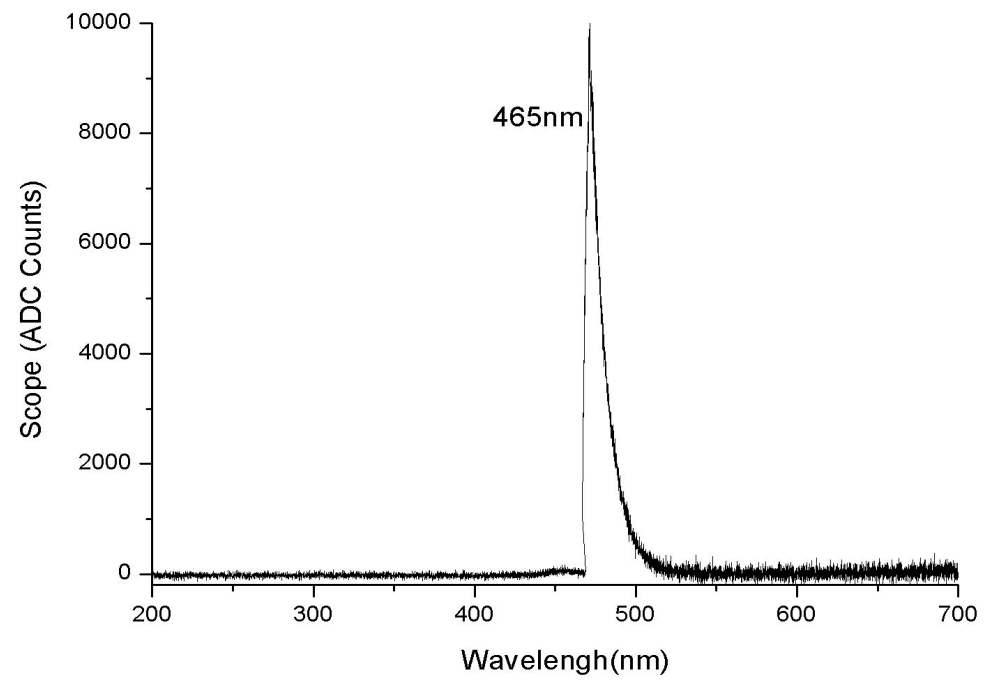

Figure S47. The spectral information of the commercially available blue LEDs light source used in experiments measured by AvaSpec-3648-2 Fiber Optic Spectrometer.<smiles>CSc1ccccc1</smiles>

MPS<smiles>CSc1ccc(Br)cc1</smiles>

BMS<smiles>CSc1ccc(C)cc1</smiles>

PMS<smiles>CSc1ccc([N+](=O)[O-])cc1</smiles>

MNS<smiles>COc1ccc(SC)cc1</smiles>

OMS

Supplementary Scheme 2. The sulfide substrates scope selected for photooxidation. 


\subsection{The photocatalysis performance of capsule 2:}

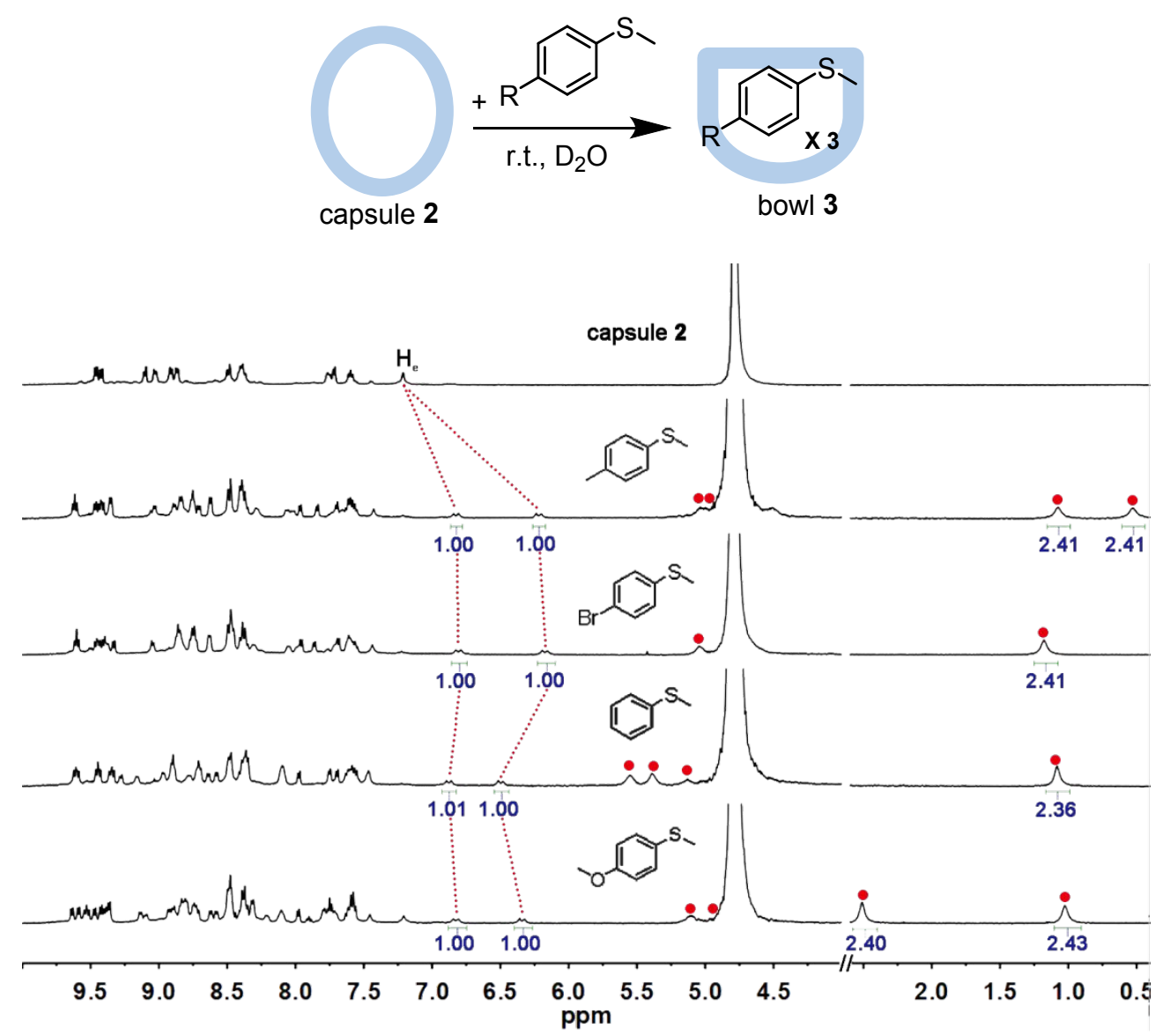

Figure S48. ${ }^{1} \mathrm{H}$ NMR spectra $\left(400 \mathrm{~Hz}, \mathrm{D}_{2} \mathrm{O}, 298 \mathrm{~K}\right)$ of the encapsulation experiments of capsule 2 toward extended substrates and structure transformation from capsule $\mathbf{2}$ to bowl $\mathbf{3}$ when adding different substrates. The 1:3 binding model was confirmed based on integration ratios. $(\bullet=$ different substrate signals) 

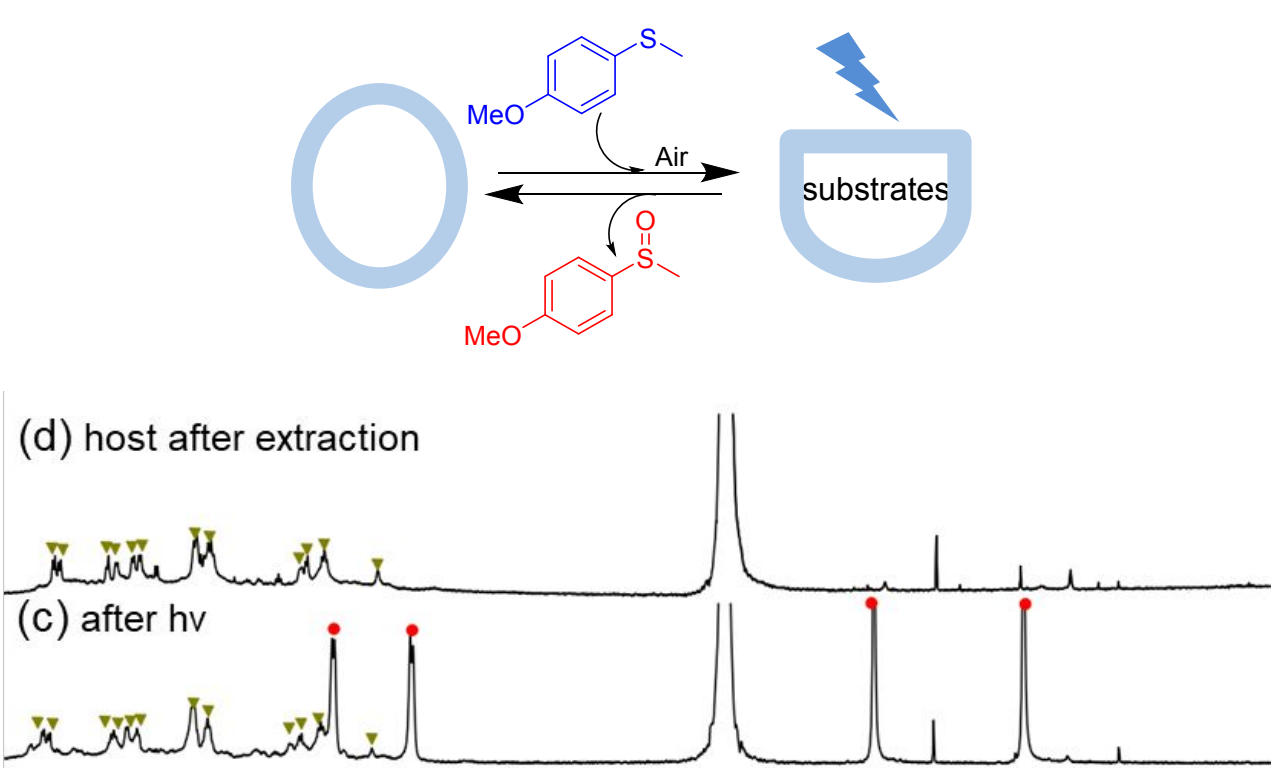

(b) oms $\subset$ bowl 3

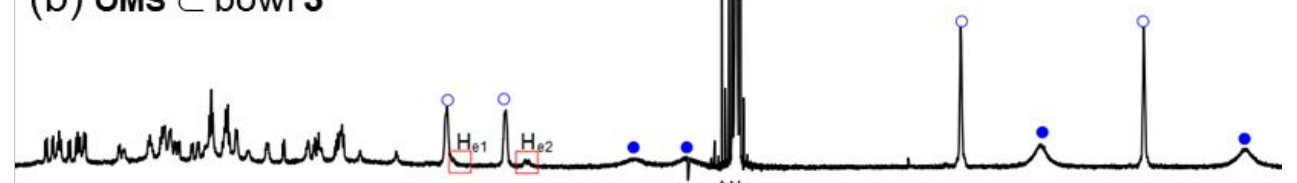

(a) capsule 2

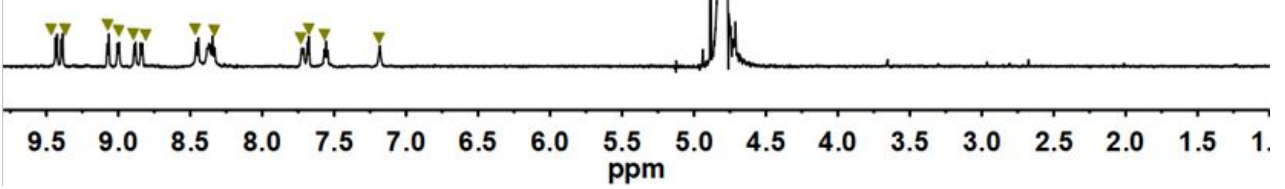

Figure S49. The trace of the photooxidation process. ${ }^{1} \mathrm{H}$ NMR spectra $\left(400 \mathrm{~Hz}, \mathrm{D}_{2} \mathrm{O}, 298 \mathrm{~K}\right)$ of $1 \mathrm{mM}$ capsule 2 (a); after adding 10 eq. OMS into a $1 \mathrm{mM}$ solution of capsule 2, transformed to bowl within 5 min (b); and after light irradiation $6 \mathrm{~h}$ at air and r.t. (c) host after full extraction with $\mathrm{CHCl}_{3}$ (d). The signals of capsule 2 were labeled with $\Delta$. The signals of OMS and product OMSO are represented by $(\bullet)$ and $(\bullet)$, respectively. 


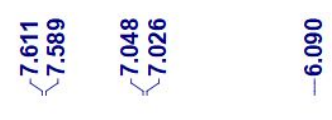 \\ 艦

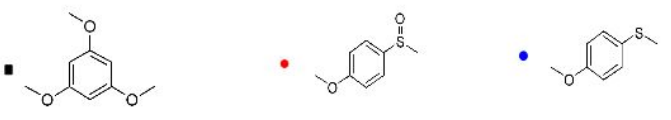

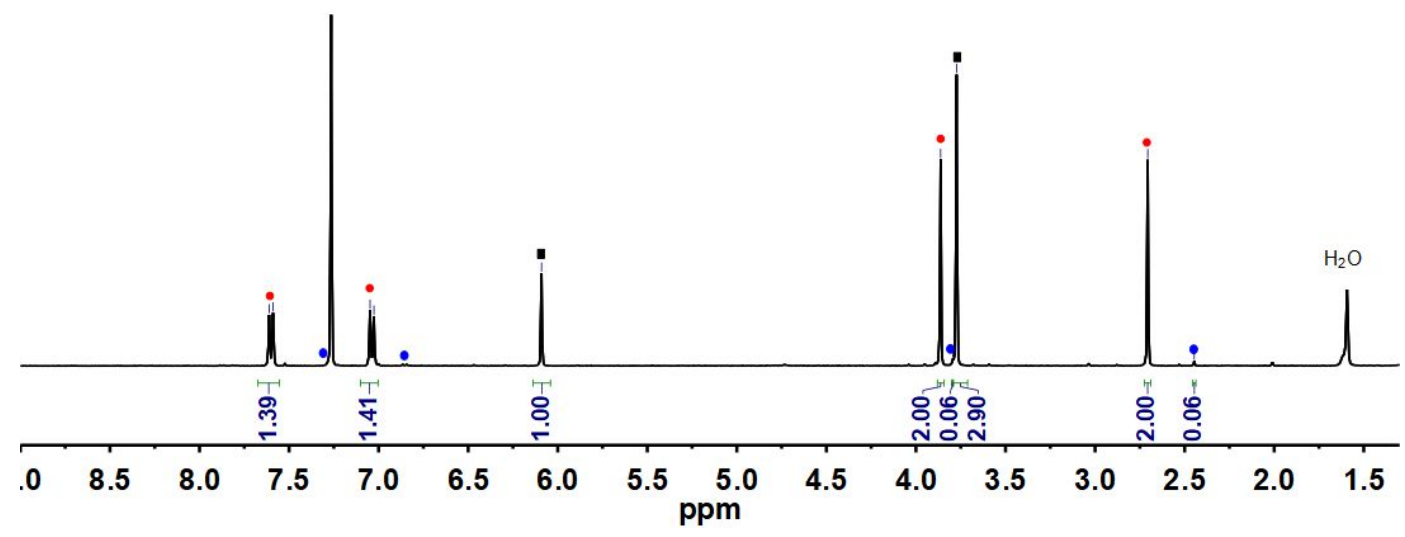

Figure S50. ${ }^{1} \mathrm{H}$ NMR spectra $\left(400 \mathrm{MHz}, 298 \mathrm{~K}, \mathrm{CDCl}_{3}\right)$ of photooxidation product of OMS, with the catalysis capsule 2 (10 mol\% equiv. catalysis loading), corresponding to entry 13 in table 1 . 1,3,5-trimethoxybenzene was used as internal standard.
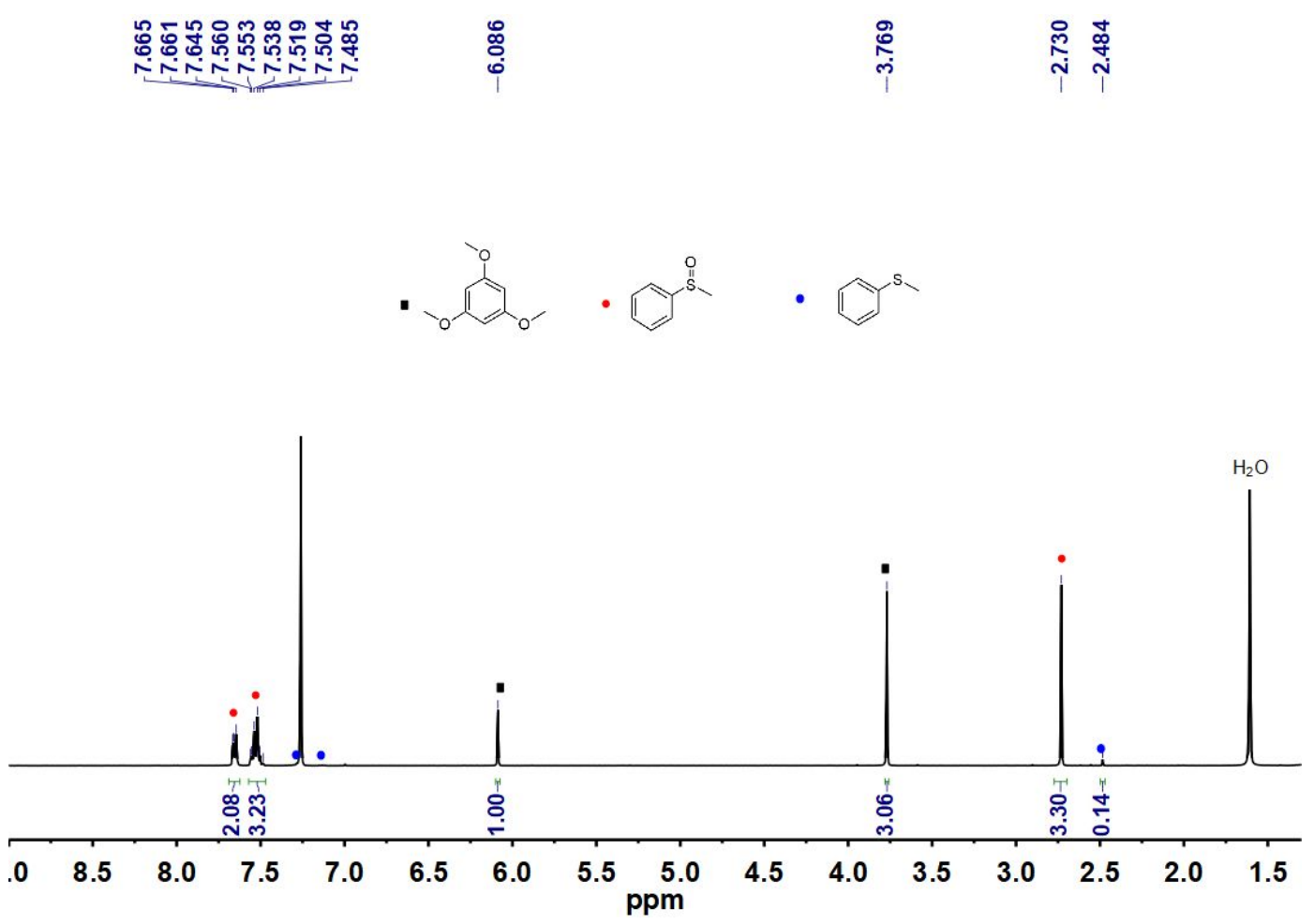

Figure S 51. ${ }^{1} \mathrm{H}$ NMR spectra $\left(400 \mathrm{MHz}, 298 \mathrm{~K}, \mathrm{CDCl}_{3}\right.$ ) of photooxidation product of MPS, with the catalysis capsule 2 (5 mol\% equiv. catalysis loading), corresponding to entry 1 in table 1 . 1,3,5-trimethoxybenzene was used as internal standard. 

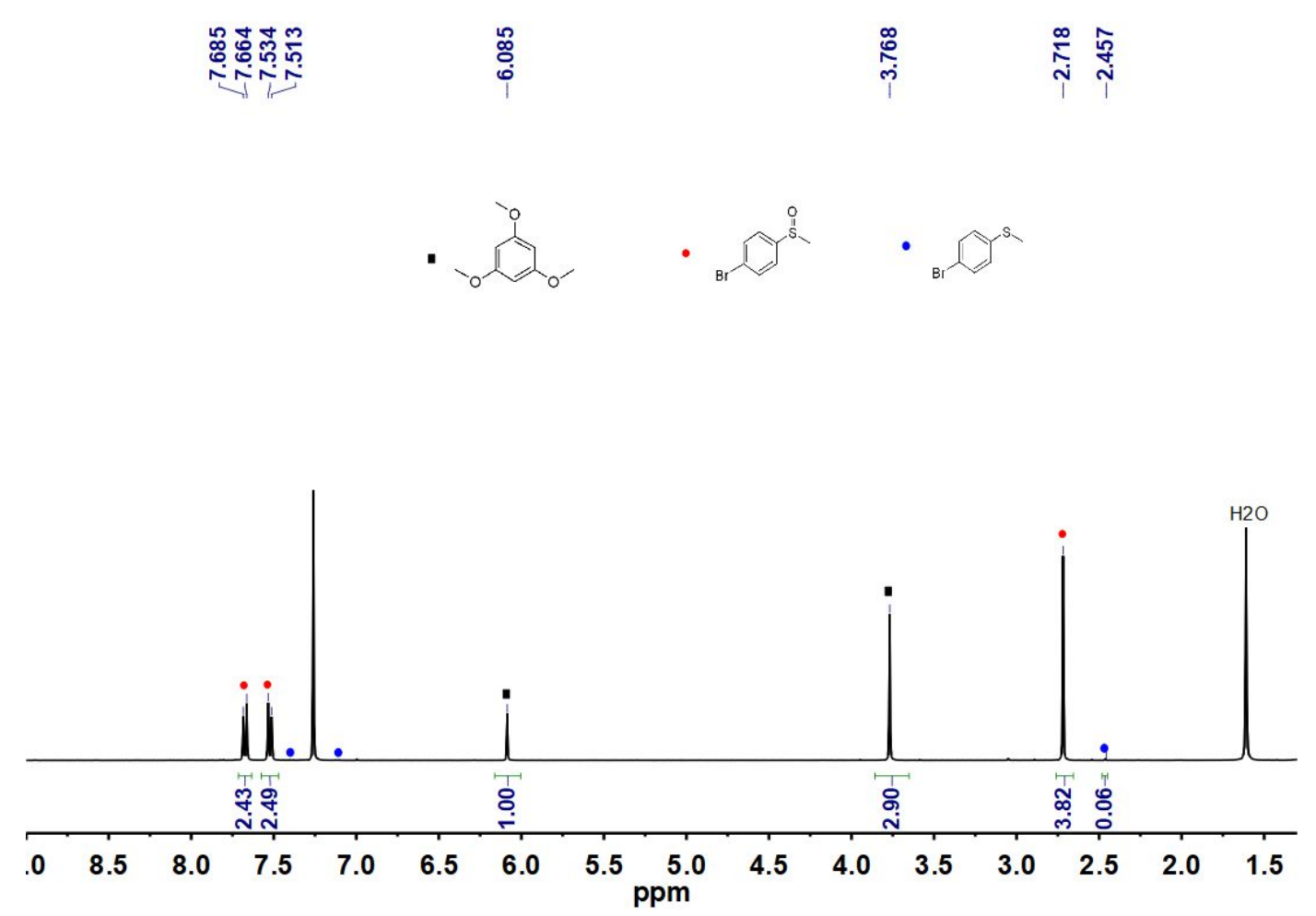

Figure S52. ${ }^{1} \mathrm{H}$ NMR spectra $\left(400 \mathrm{MHz}, 298 \mathrm{~K}, \mathrm{CDCl}_{3}\right.$ ) of photooxidation product of BMS, with the catalysis capsule 2 (5 mol\% equiv. catalysis loading), corresponding to entry 4 in table 1 . 1,3,5-trimethoxybenzene was used as internal standard.
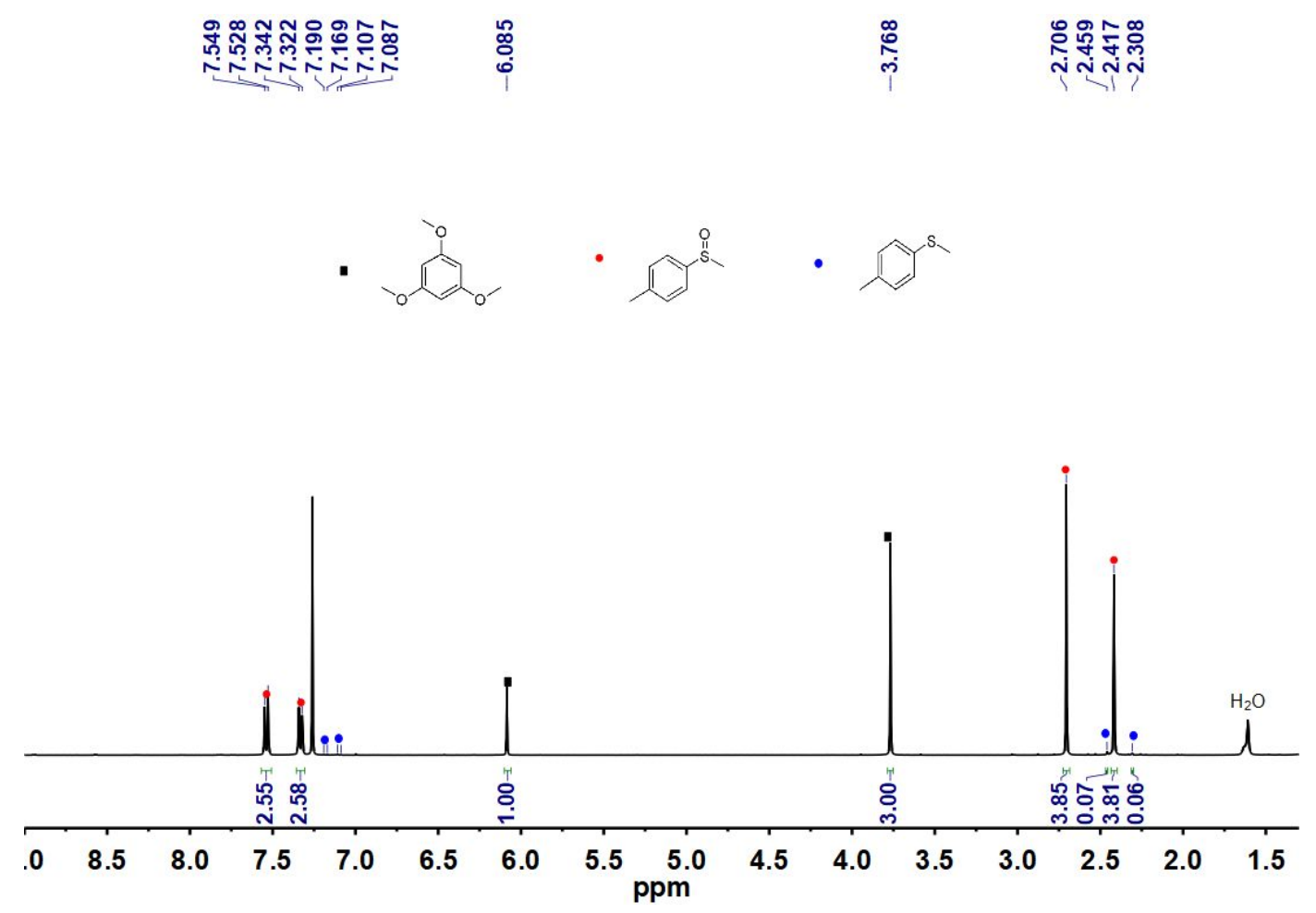

Figure S53. ${ }^{1} \mathrm{H}$ NMR spectra $\left(600 \mathrm{MHz}, 298 \mathrm{~K}, \mathrm{CDCl}_{3}\right)$ of photooxidation product of PMS, with the catalysis capsule 2 ( $5 \mathrm{~mol} \%$ equiv. catalysis loading), corresponding to entry 7 in table 1. 1,3,5-trimethoxybenzene was used as internal standard. 
<smiles>COc1cc(OC)cc(OC)c1</smiles><smiles>CCC1CCC([N+](=O)[O-])CC1</smiles>

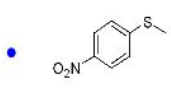

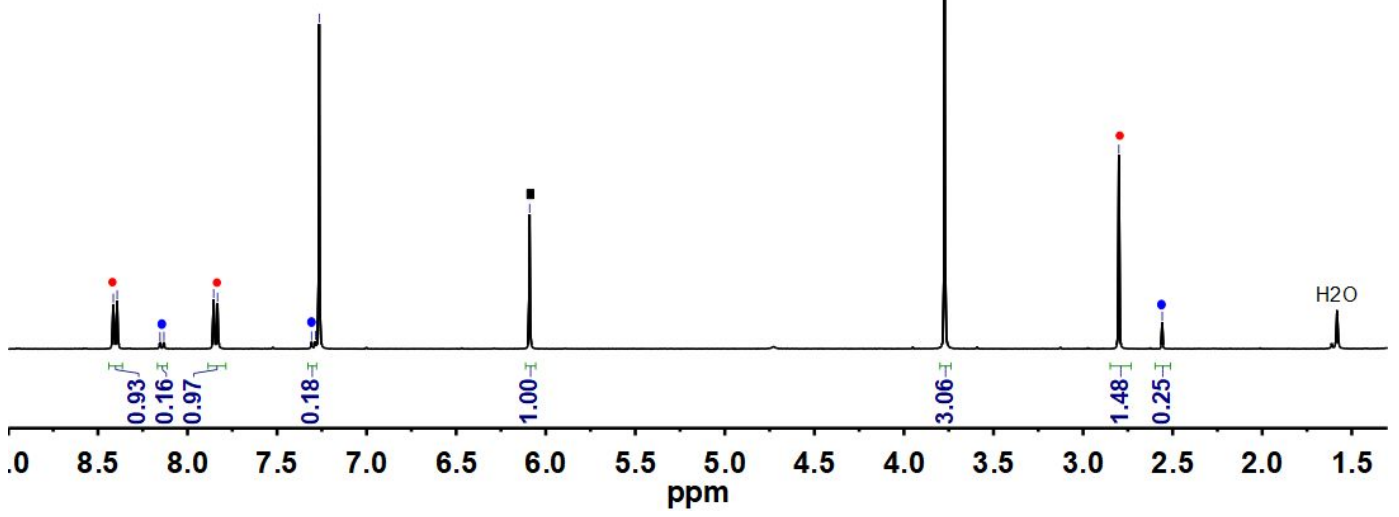

Figure 54. ${ }^{1} \mathrm{H}$ NMR spectra $\left(400 \mathrm{MHz}, 298 \mathrm{~K}, \mathrm{CDCl}_{3}\right)$ of photooxidation product of MNS, with the catalysis capsule 2 (10 mol\% equiv. catalysis loading), corresponding to entry 10 in table 1. 1,3,5-trimethoxybenzene was used as internal standard.

\subsection{The photocatalysis performance of cage 1:}

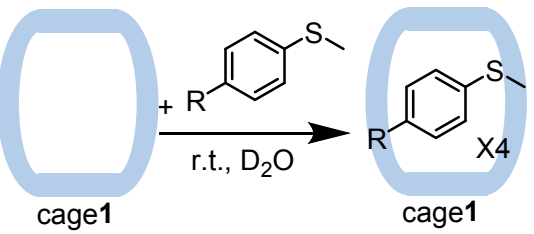




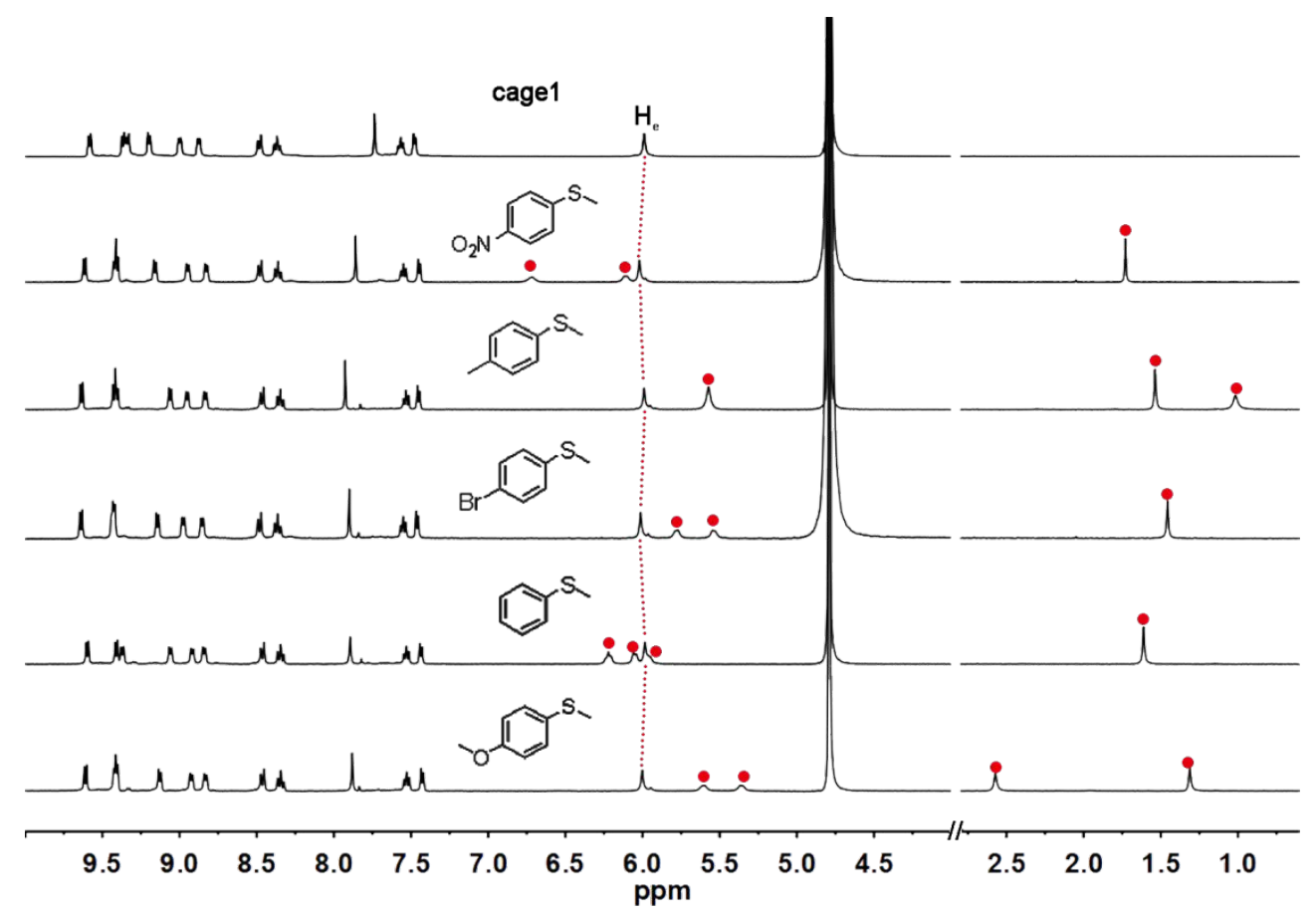

Figure S55. ${ }^{1} \mathrm{H}$ NMR spectra $\left(400 \mathrm{~Hz}, \mathrm{D}_{2} \mathrm{O}, 298 \mathrm{~K}\right)$ of the encapsulation experiments of cage 1 toward extended substrates when adding different substrates, showing no substrates-adaptive structure transformation. $(\bullet=$ different substrate signals $)$

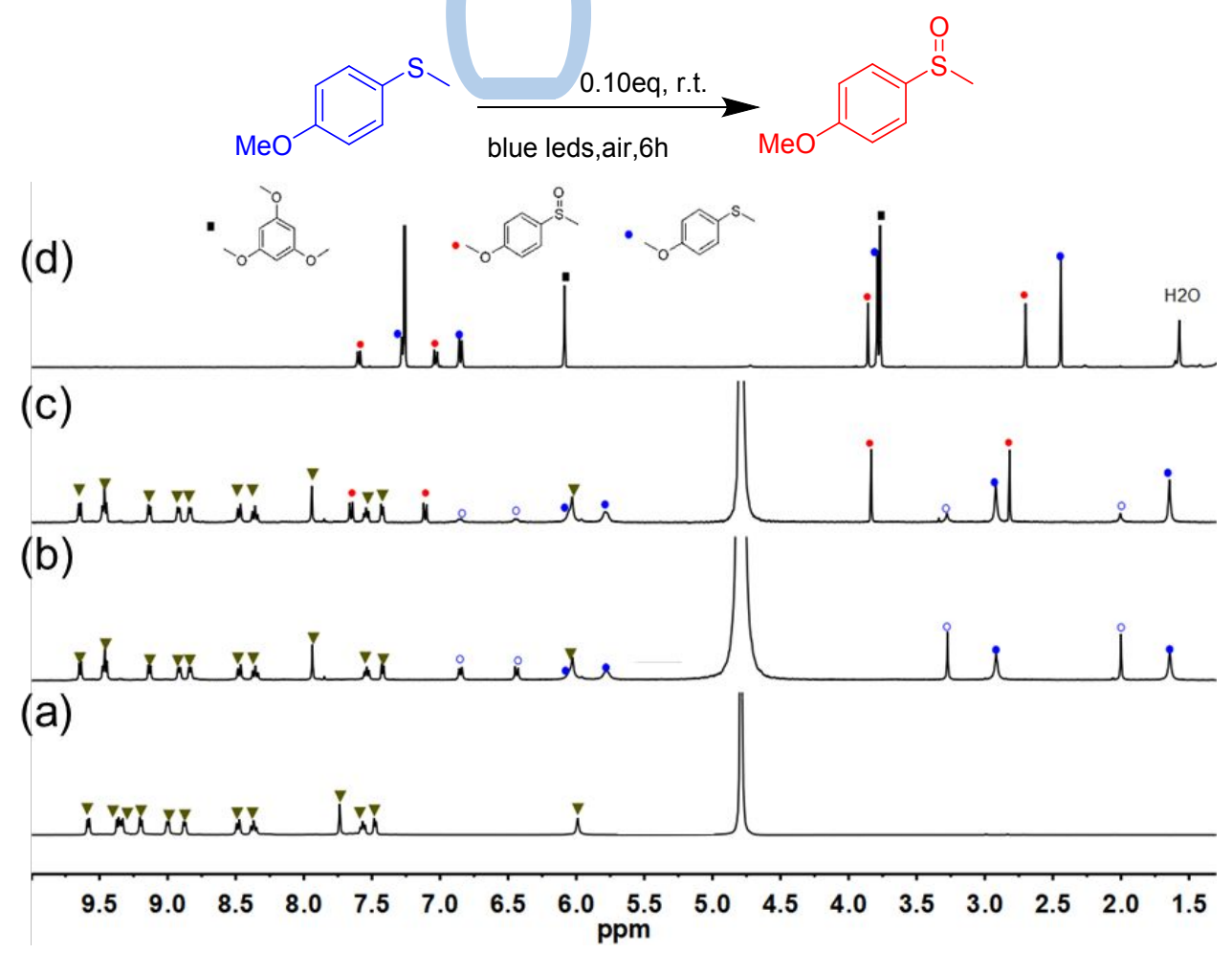

Figure S56. The trace of the photooxidation process. ${ }^{1} \mathrm{H}$ NMR spectra (400 MHz, $\mathrm{D}_{2} \mathrm{O}, 298 \mathrm{~K}$ ) of 1 $\mathrm{mM}$ cage 1 (a); inclusion complex by adding 10 eq. OMS into a $1 \mathrm{mM}$ solution of cage $\mathbf{1}$ (b); and after light irradiation $6 \mathrm{~h}$ at air and r.t. (c); product after extraction with $\mathrm{CHCl}_{3}$ (d). The signals of cage 1 were labeled with $\boldsymbol{\Delta}$. The signals of OMS and product OMSO are represented by $(\bullet)$ and $(\bullet)$, 
respectively.
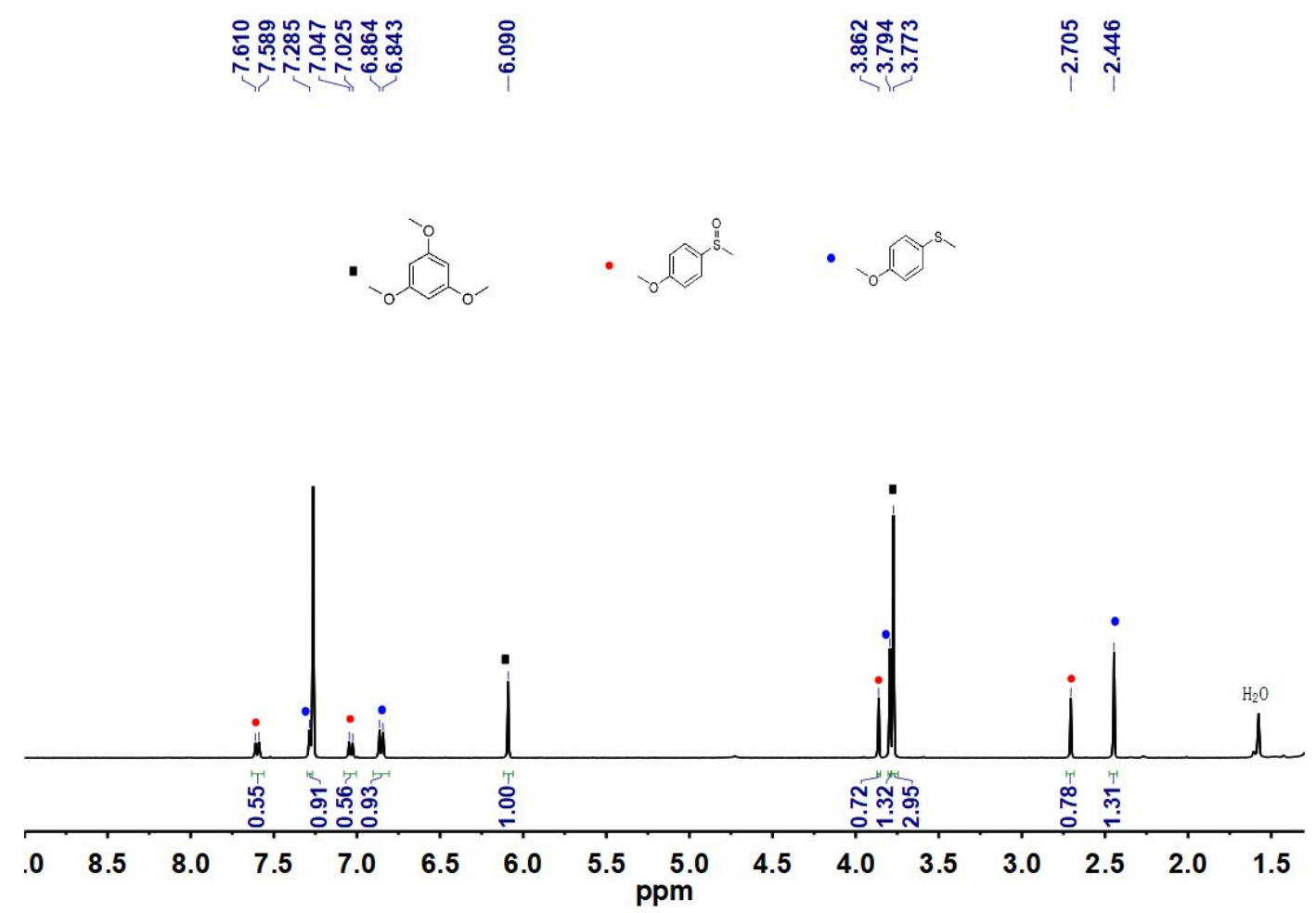

Figure S57. ${ }^{1} \mathrm{H}$ NMR spectra $\left(400 \mathrm{MHz}, 298 \mathrm{~K}, \mathrm{CDCl}_{3}\right)$ of photooxidation product of OMS, with the catalysis cage 1 (10 mol\% equiv. catalysis loading), corresponding to entry 14 in table 1 . 1,3,5-trimethoxybenzene was used as internal standard.
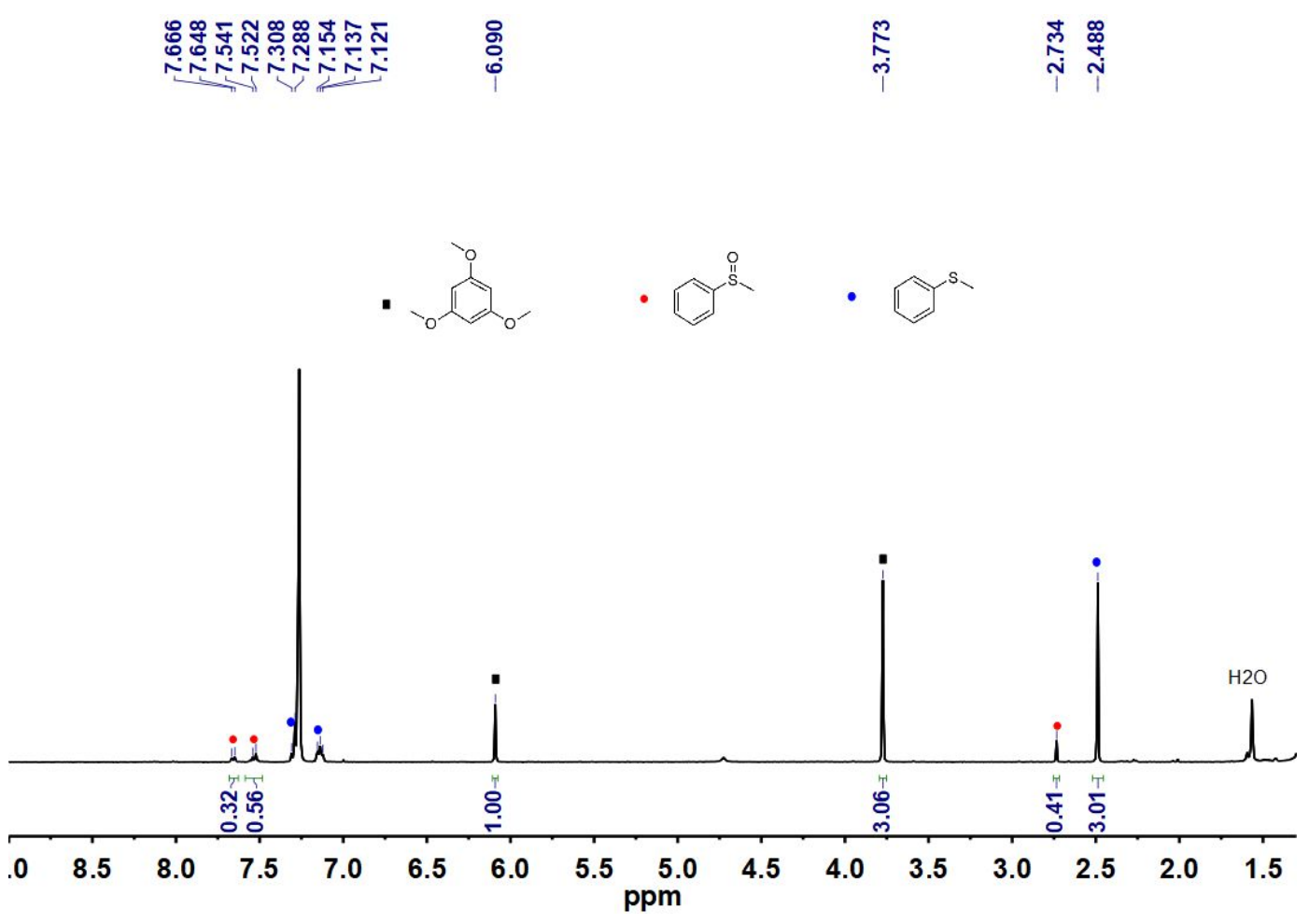

Figure S58. ${ }^{1} \mathrm{H}$ NMR spectra ( $400 \mathrm{MHz}, 298 \mathrm{~K}, \mathrm{CDCl}_{3}$ ) of photooxidation product of MPS, with the catalysis cage 1 (5 mol\% equiv. catalysis loading), corresponding to entry 2 in table 1. 
1,3,5-trimethoxybenzene was used as internal standard.
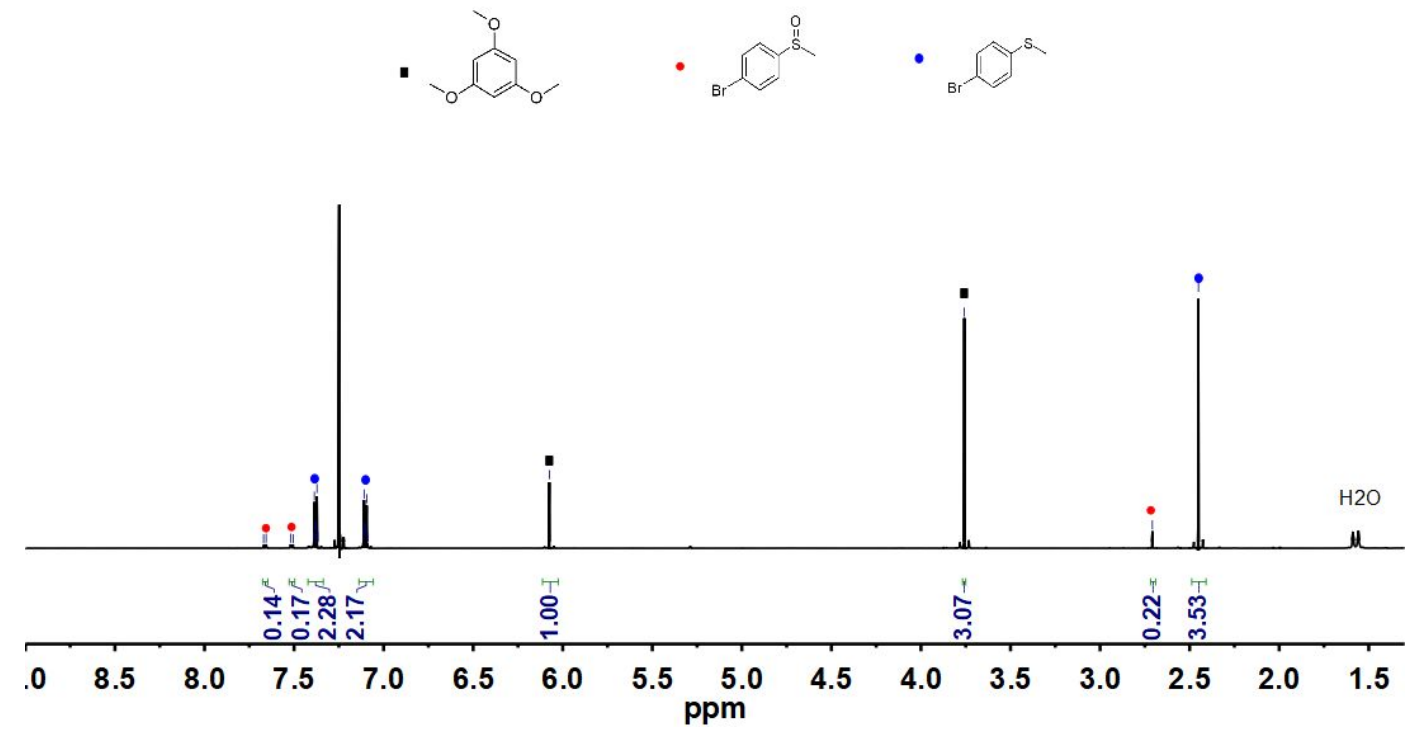

Figure S59. ${ }^{1} \mathrm{H}$ NMR spectra $\left(600 \mathrm{MHz}, 298 \mathrm{~K}, \mathrm{CDCl}_{3}\right)$ of photooxidation product of BMS, with the catalysis cage 1 ( 5 mol\% equiv. catalysis loading), corresponding to entry 5 in table 1 . 1,3,5-trimethoxybenzene was used as internal standard.

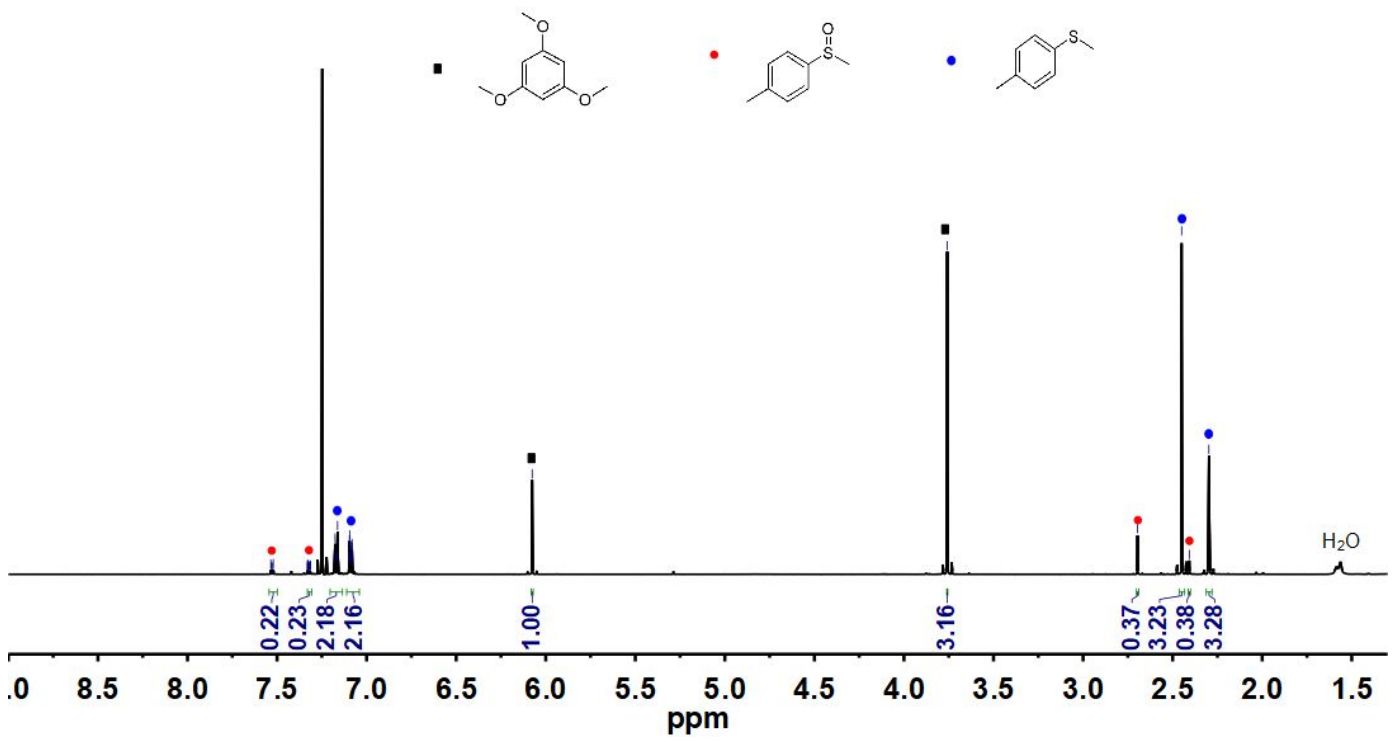

Figure S60. ${ }^{1} \mathrm{H}$ NMR spectra $\left(600 \mathrm{MHz}, 298 \mathrm{~K}, \mathrm{CDCl}_{3}\right)$ of photooxidation product of PMS, with the catalysis cage 1 ( 5 mol\% equiv. catalysis loading), corresponding to entry 8 in table 1 . 1,3,5-trimethoxybenzene was used as internal standard. 

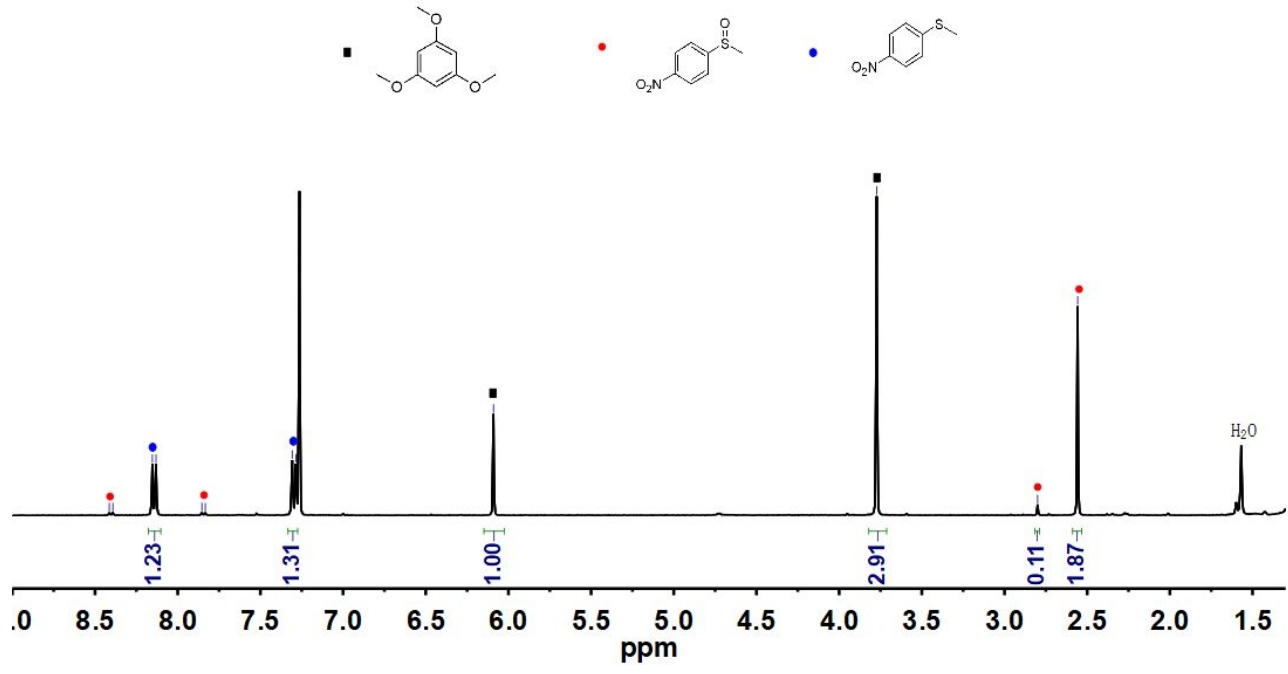

Figure S61. ${ }^{1} \mathrm{H}$ NMR spectra (400 MHz, $298 \mathrm{~K}, \mathrm{CDCl}_{3}$ ) of photooxidation product of MNS, with the catalysis cage 1 (10 mol\% equiv. catalysis loading), corresponding to entry 11 in table 1 . 1,3,5-trimethoxybenzene was used as internal standard.

\subsection{Control experiments and proposed mechanism:}

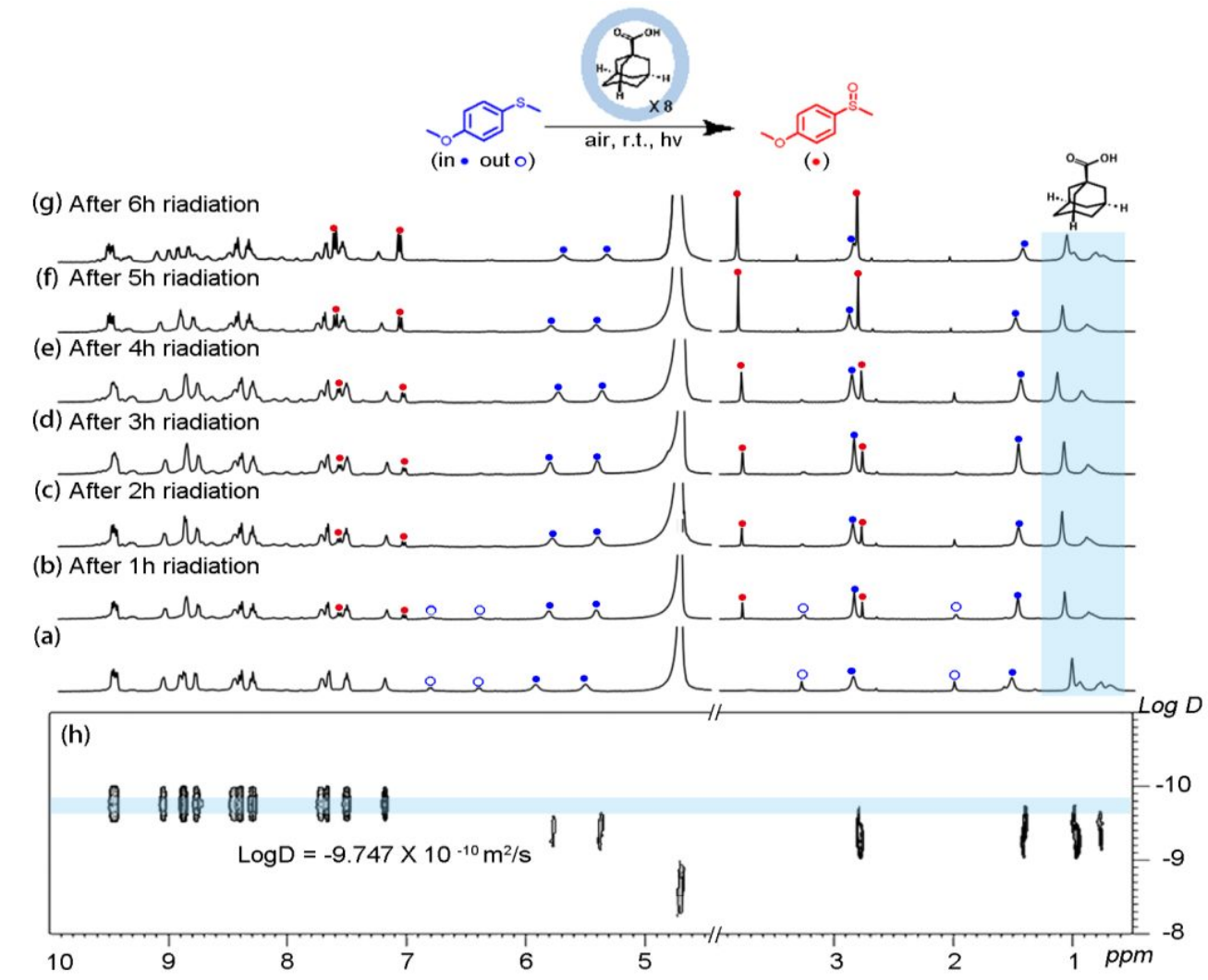

Figure S62. (a-g) Partial ${ }^{1} \mathrm{H}$ NMR tracked spectra $\left(400 \mathrm{MHz}, 298 \mathrm{~K}, \mathrm{D}_{2} \mathrm{O}\right)$ of photooxidation process 
with $\mathbf{A D C} \mathbf{C}_{8} \subset \mathbf{2}$ as the catalyst; (h) ${ }^{1} \mathrm{H}$ DOSY spectrum of $\mathbf{A D C} \mathbf{C}_{8} \subset \mathbf{2}$ after adding 10 eq. of $\mathbf{O M S}$.

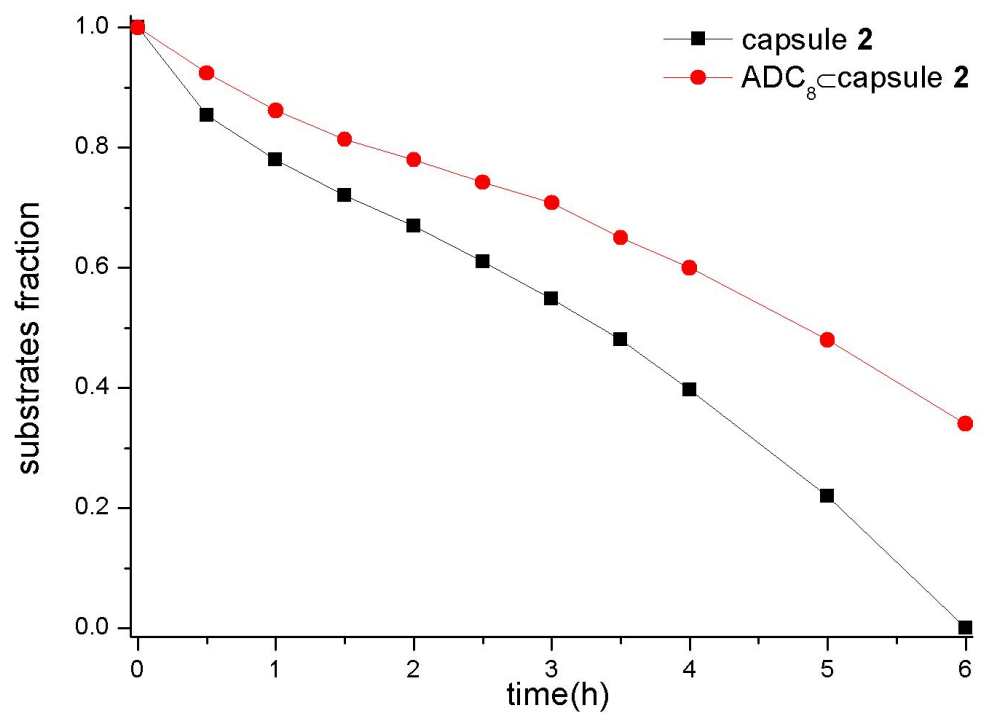

Figure S63. The comparison of the conversions for the photooxidation of OMS (10 eq., under $6 \mathrm{~W}$ 465-nm LEDs) with either capsule $\mathbf{2}$ or $\mathbf{A D C}_{8} \subset \mathbf{2}$ as the catalysts.

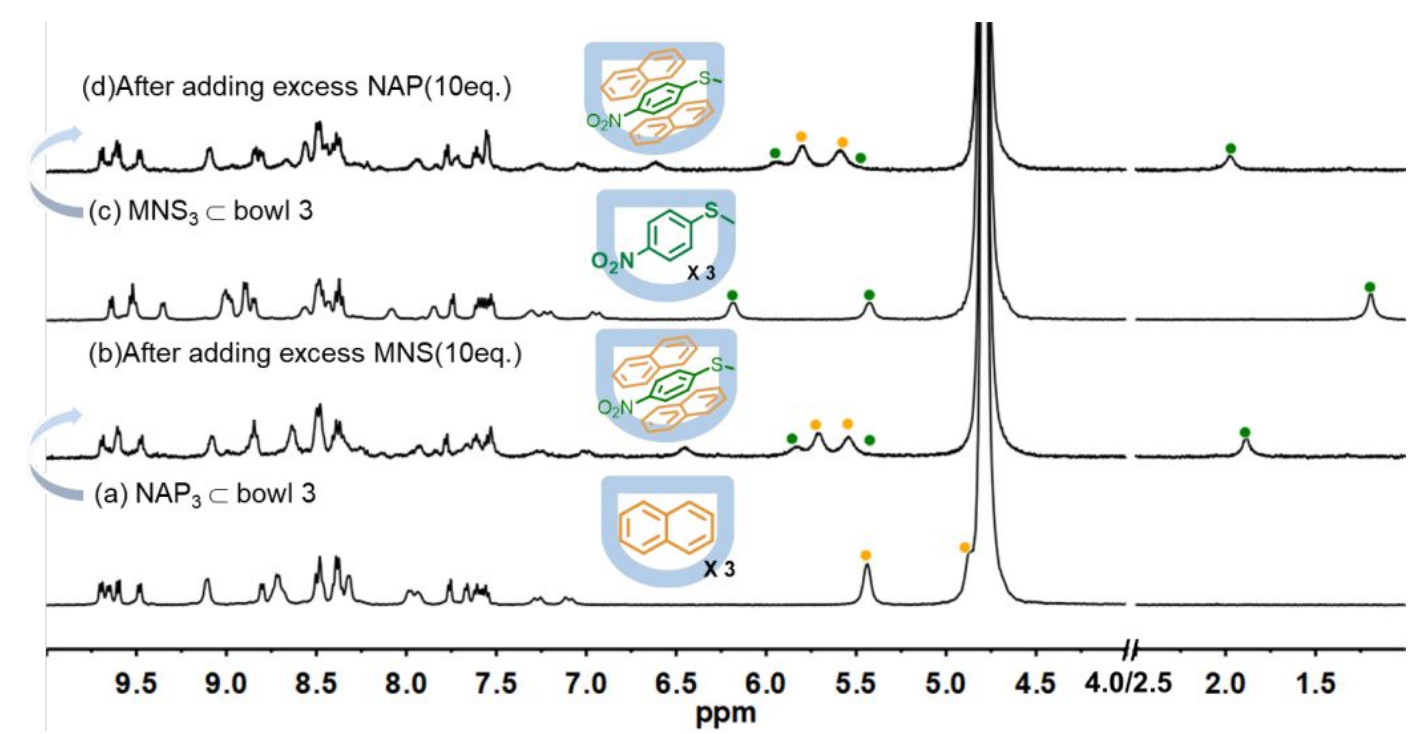

Figure S64. ${ }^{1} \mathrm{H}$ NMR spectra $\left(400 \mathrm{MHz}, 298 \mathrm{~K}, \mathrm{D}_{2} \mathrm{O}\right.$ ) of competitive encapsulation experiments of naphthalene (NAP) and methyl(4-nitrophenyl)sulfane (MNS). 


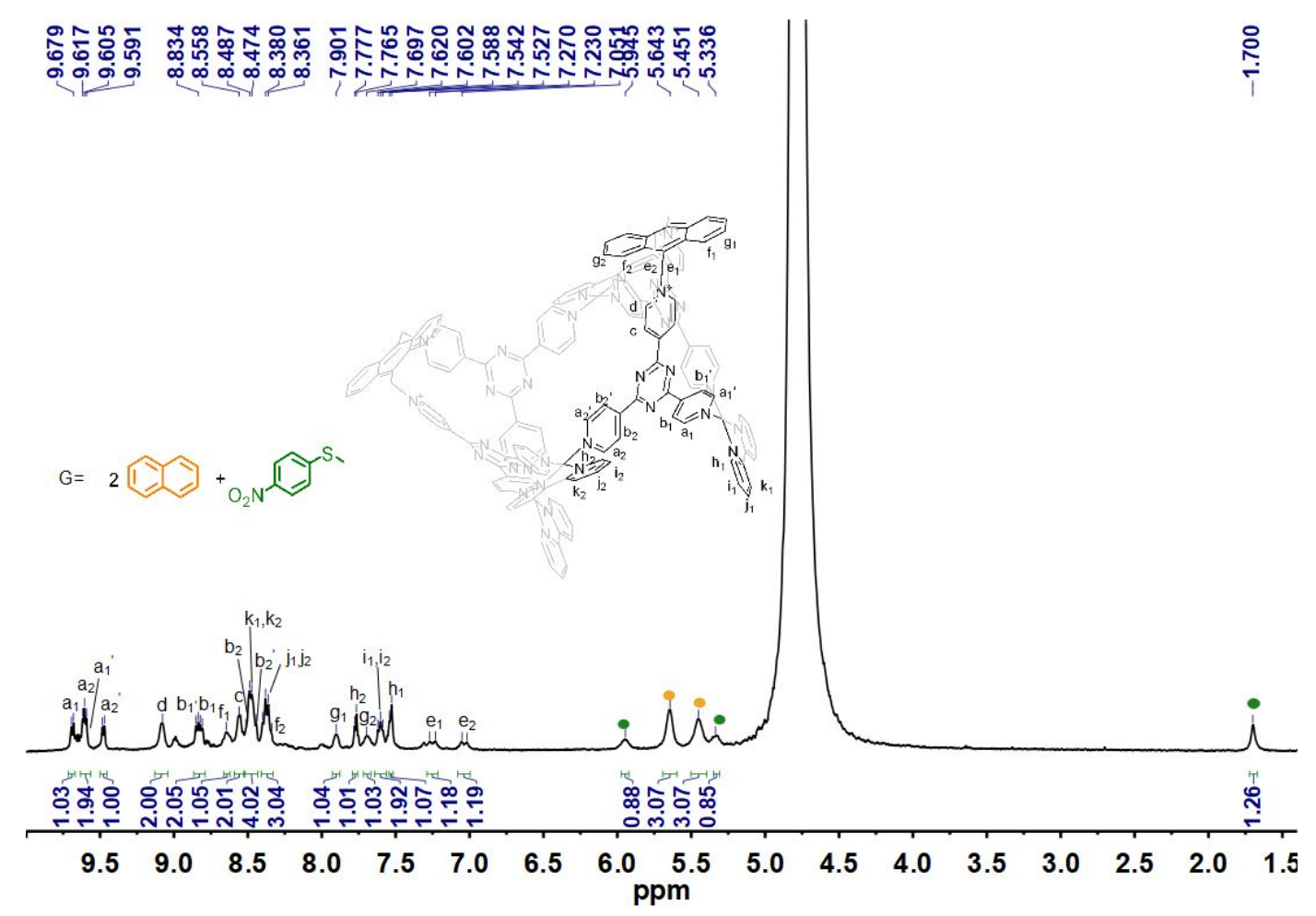

Figure S65. ${ }^{1} \mathrm{H}$ NMR spectrum $\left(400 \mathrm{MHz}, \mathrm{D}_{2} \mathrm{O}, 298 \mathrm{~K}\right)$ of ternary inclusion complex $\left(\mathbf{N A P}_{2}+\mathbf{M N S}\right) \subset$ $\mathbf{3}$ based on integration ratios. Guest signals are labeled with circles in a different color.

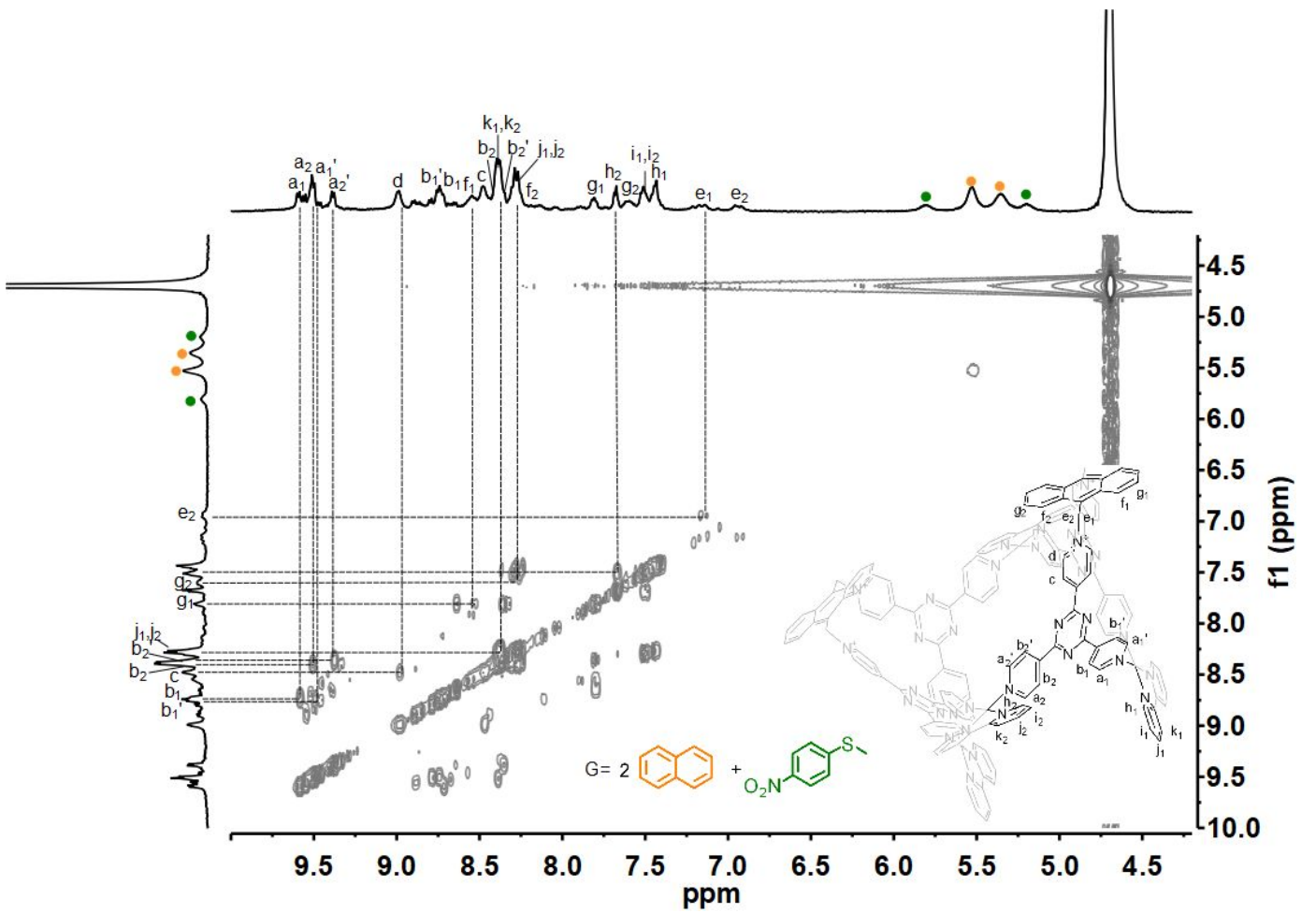

Figure S66. ${ }^{1} \mathrm{H}-{ }^{1} \mathrm{H}$ COSY NMR spectrum (400 MHz, $\mathrm{D}_{2} \mathrm{O}, 298 \mathrm{~K}$ ) of ternary inclusion complex $\left(\mathbf{N A P}_{2}+\mathbf{M N S}\right) \subset \mathbf{3}$. Guest signals are labeled with circles in a different color. 


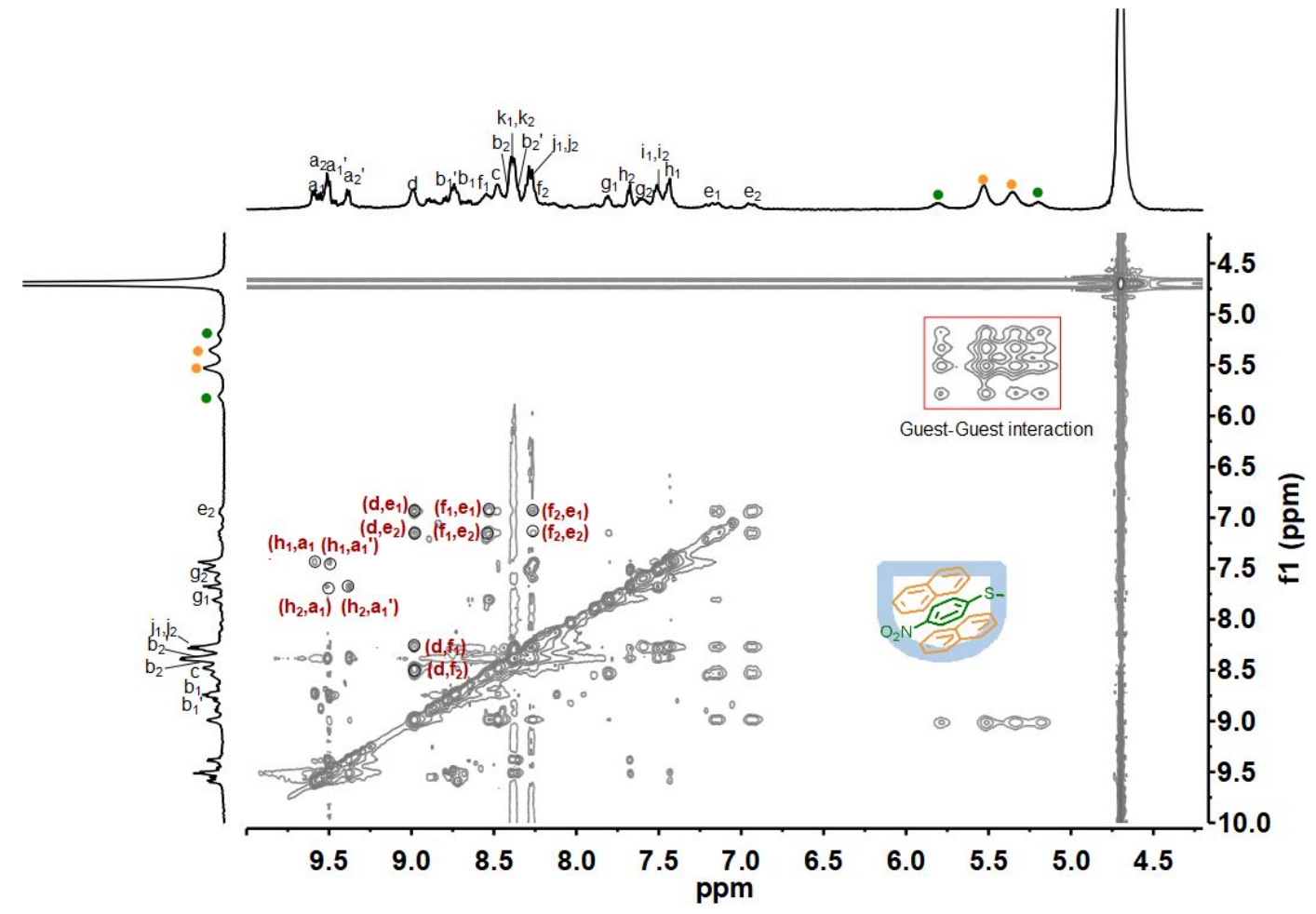

Figure S67. ${ }^{1} \mathrm{H}-{ }^{1} \mathrm{H}$ NOESY NMR spectrum $\left(400 \mathrm{MHz}, \mathrm{D}_{2} \mathrm{O}, 298 \mathrm{~K}\right)$ of ternary inclusion complex $\left(\mathbf{N A P}_{2}+\mathbf{M N S}\right) \subset \mathbf{3}$. Guest signals are labeled with circles in a different color.

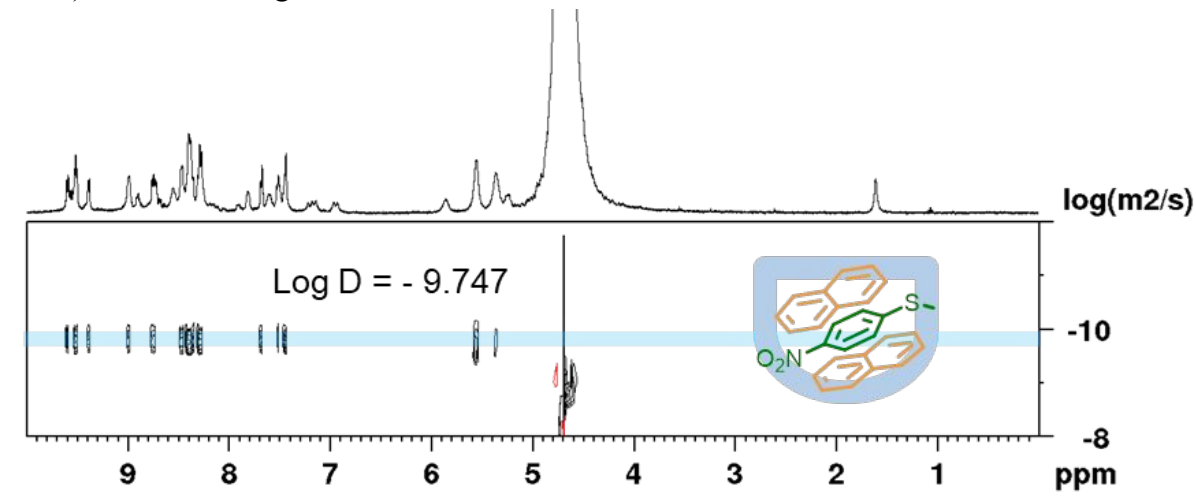

Figure S68. ${ }^{1} \mathrm{H}$ DOSY spectrum $\left(400 \mathrm{MHz}, \mathrm{D}_{2} \mathrm{O}, 298 \mathrm{~K}\right)$ of ternary inclusion complex $\left(\mathbf{N A P}_{2}+\mathbf{M N S}\right)$ $\subset$ 3. (Diffusion Constant $=1.79 \mathrm{E}^{-10} \mathrm{~m}^{2} / \mathrm{S}, \mathrm{d}=2.73 \mathrm{~nm}$ )
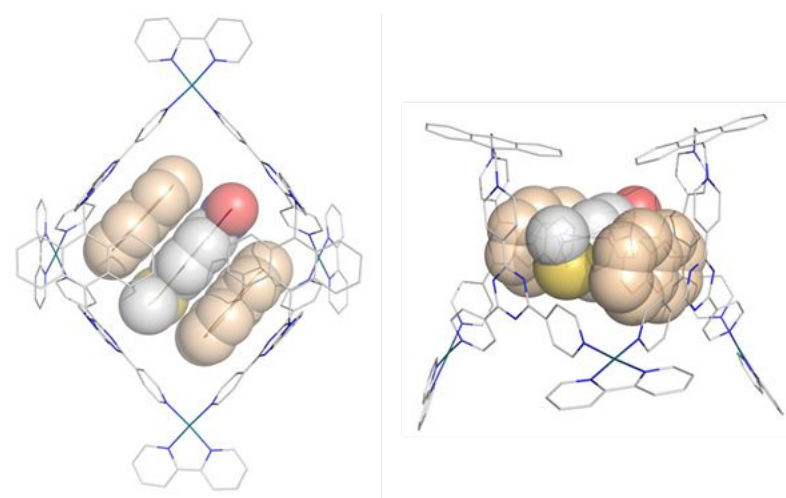

Figure S69. The proposed structure for the ternary complex of $\left(\mathbf{N A P}_{2}+\mathbf{M N S}\right) \subset \mathbf{3}$. 


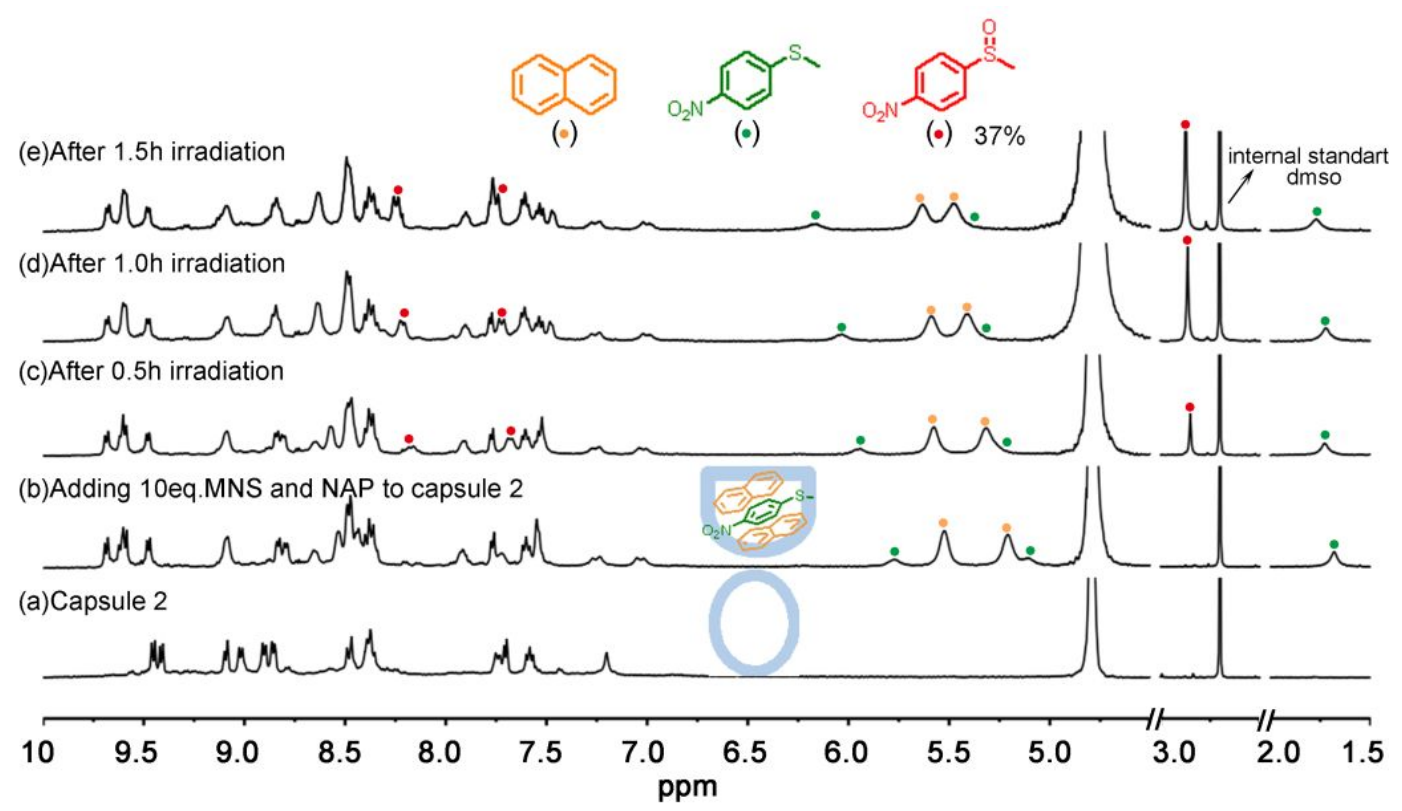

Figure S70. In-situ tracked NMR spectra of the photo-reaction in presence of naphthalene as inhibitor (with $5 \mathrm{mM}$ of DMSO as the internal standard).
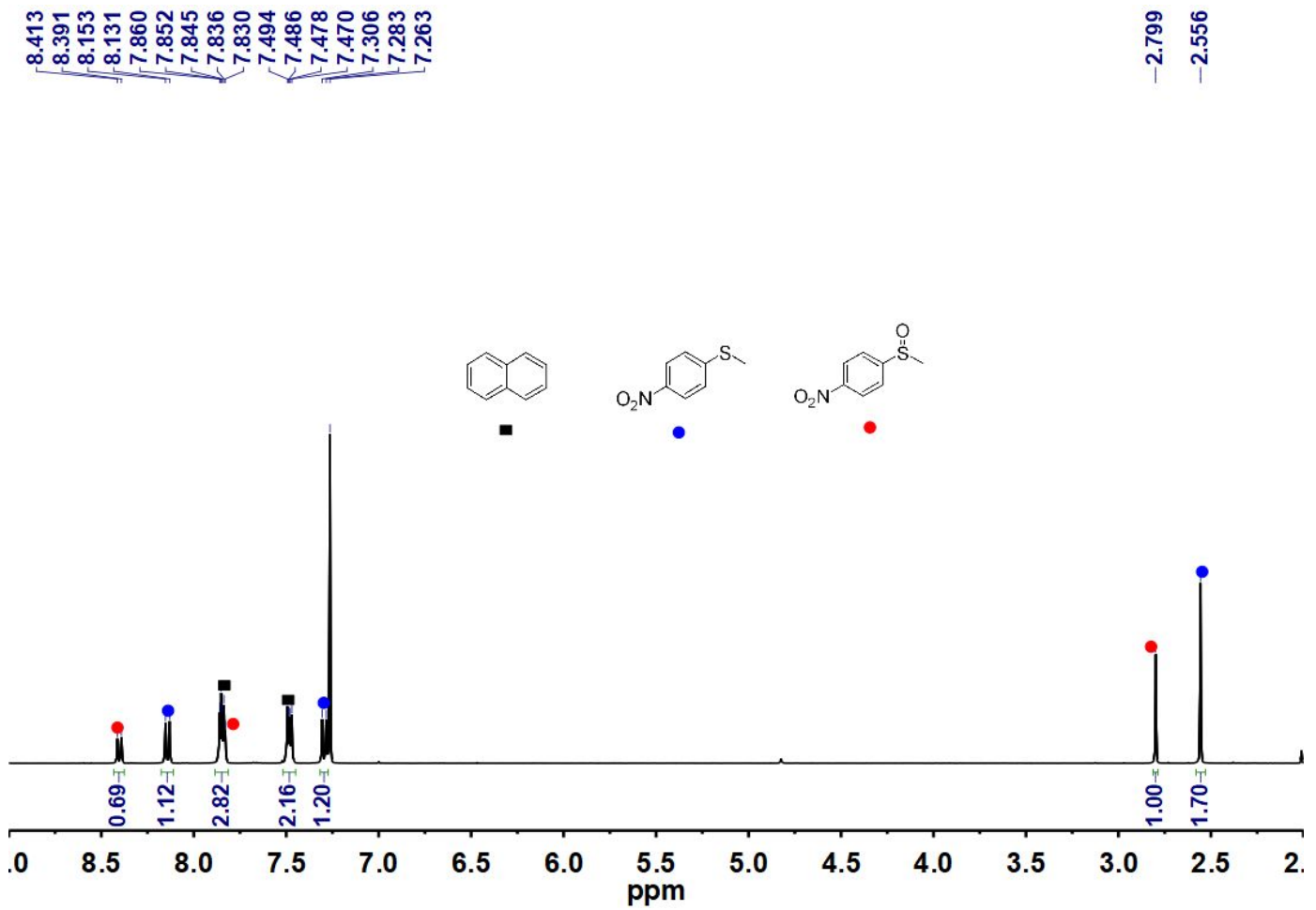

Figure S71. ${ }^{1} \mathrm{H}$ NMR spectrum ( $400 \mathrm{MHz}, 298 \mathrm{~K}, \mathrm{CDCl}_{3}$ ) of crude extracted from the reaction mixture in the presence of competitive guest naphthalene molecules (10 equiv.) as blocking reagents, a much lower yield $37 \%$ of sulfoxide. (Reaction conditions: 10 equiv. of NMS as substrate, air as oxidant, 1 $\mathrm{mM}$ of capsule 2 as catalysts, and blue LEDs irradiation for $1.5 \mathrm{~h}$.) 


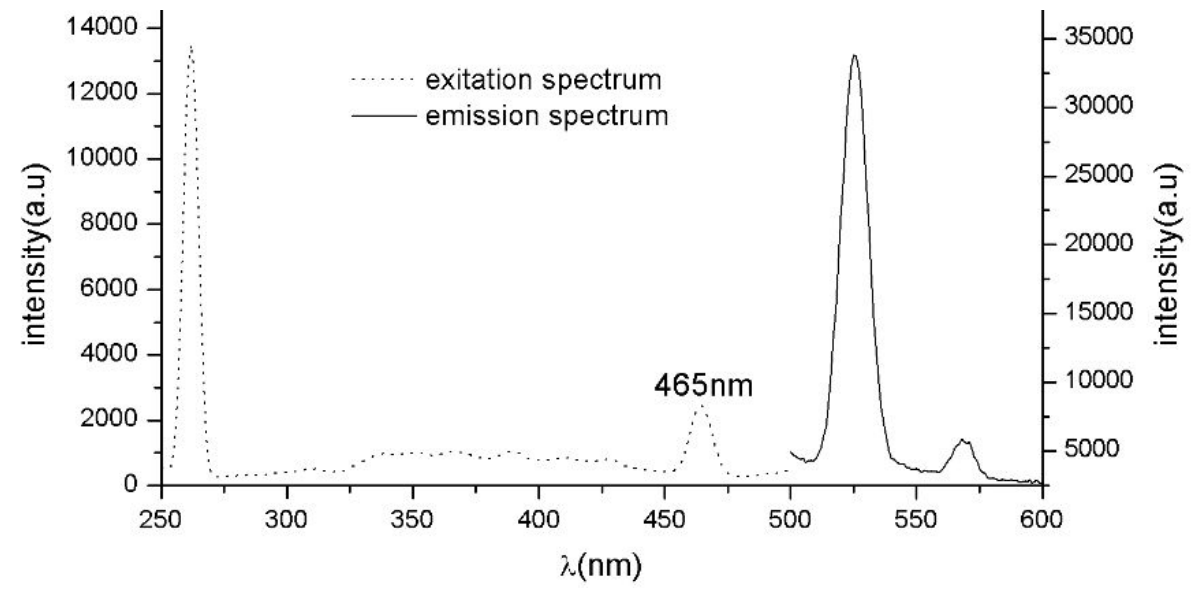

Figure S72. Excitation $\left(\lambda_{\mathrm{em}}=525 \mathrm{~nm}\right)$ and emission $\left(\lambda_{\mathrm{ex}}=465 \mathrm{~nm}\right)$ spectra for $\mathbf{O M S}_{3} \subset$ bowl 3 in $\mathrm{D}_{2} \mathrm{O}$ $\left(1 \times 10^{-2} \mathrm{mM}\right.$, slits $\left.=6.5 \mathrm{~nm}\right)$
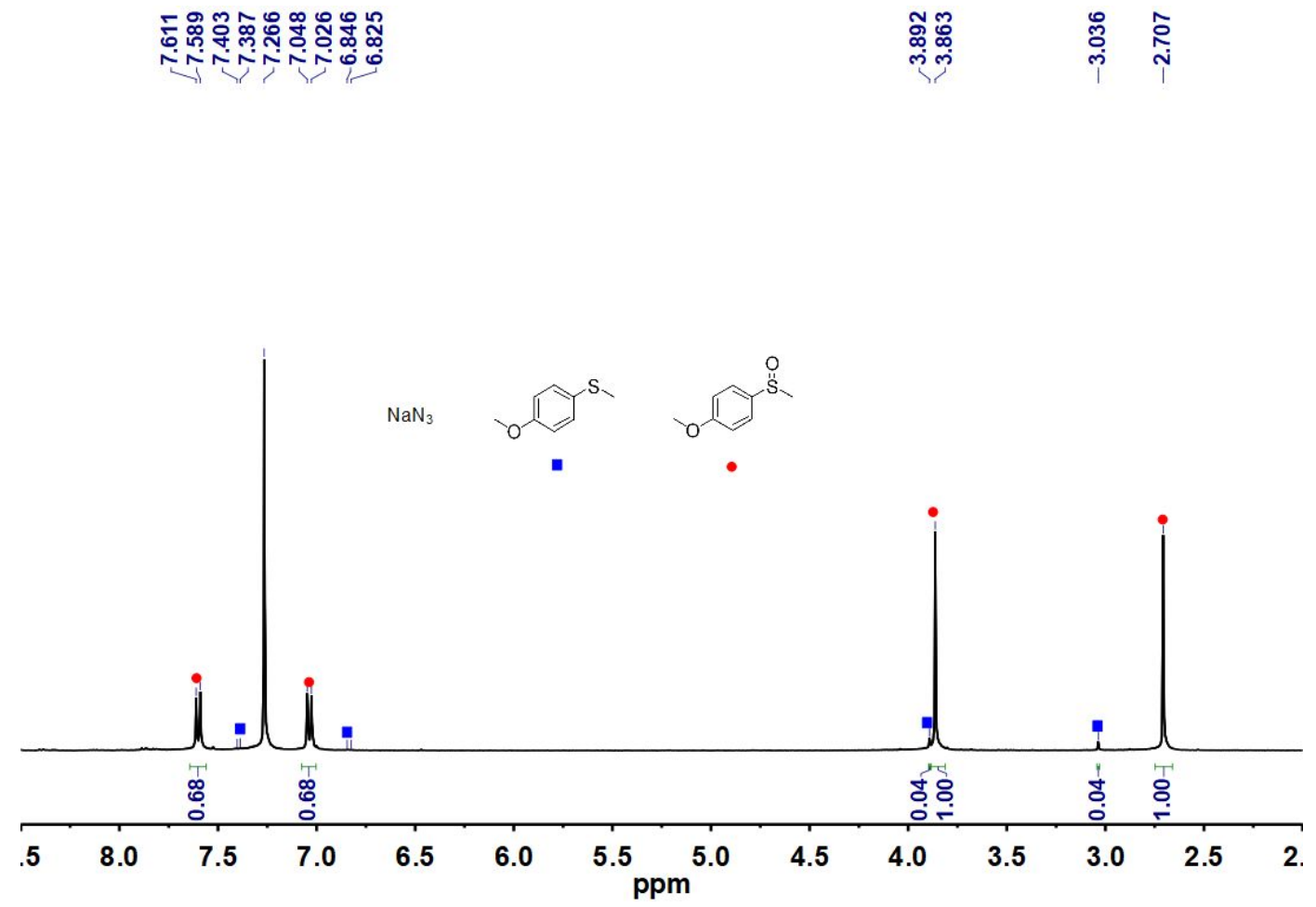

Figure S73. ${ }^{1} \mathrm{H}$ NMR spectrum ( $400 \mathrm{MHz}, 298 \mathrm{~K}, \mathrm{CDCl}_{3}$ ) of crude extracted from the reaction mixture in the presence of $\mathrm{NaN}_{3}$, which showed no negative effect on the photooxidation of OMS. (Reaction conditions: 10 eq. of OMS as substrate, air as oxidant, $1 \mathrm{mM}$ of capsule 2 as catalysts, and blue LEDs irradiation for $6 \mathrm{~h}$.) 


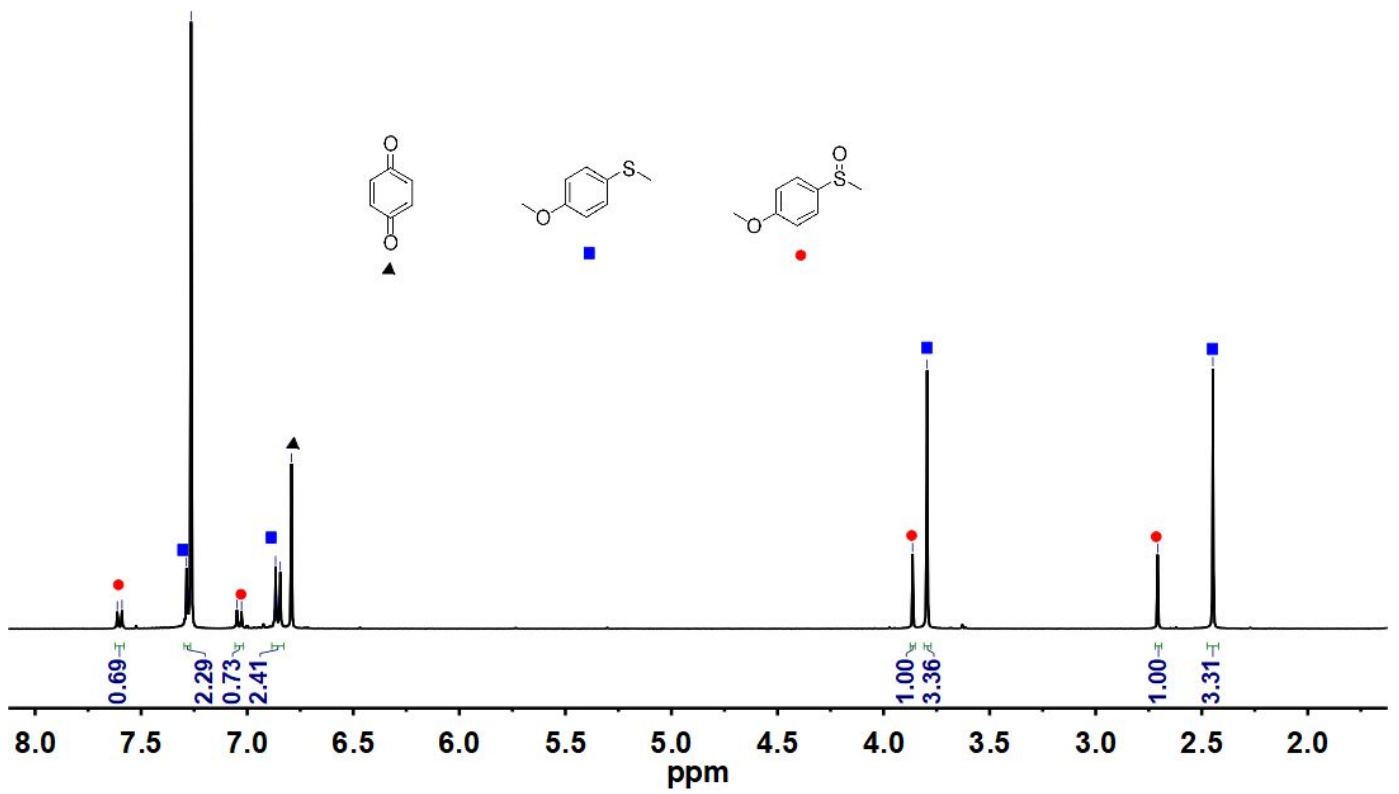

Figure S74. ${ }^{1} \mathrm{H}$ NMR spectrum ( $400 \mathrm{MHz}, 298 \mathrm{~K}, \mathrm{CDCl}_{3}$ ) of crude extracted from the reaction mixture in the presence of benzoquinone, showing an obvious drop on conversion rate. (Reaction conditions: 10 eq. of OMS as substrate, air as oxidant, $1 \mathrm{mM}$ of capsule $\mathbf{2}$ as catalysts, and blue LEDs irradiation for 6 h.)

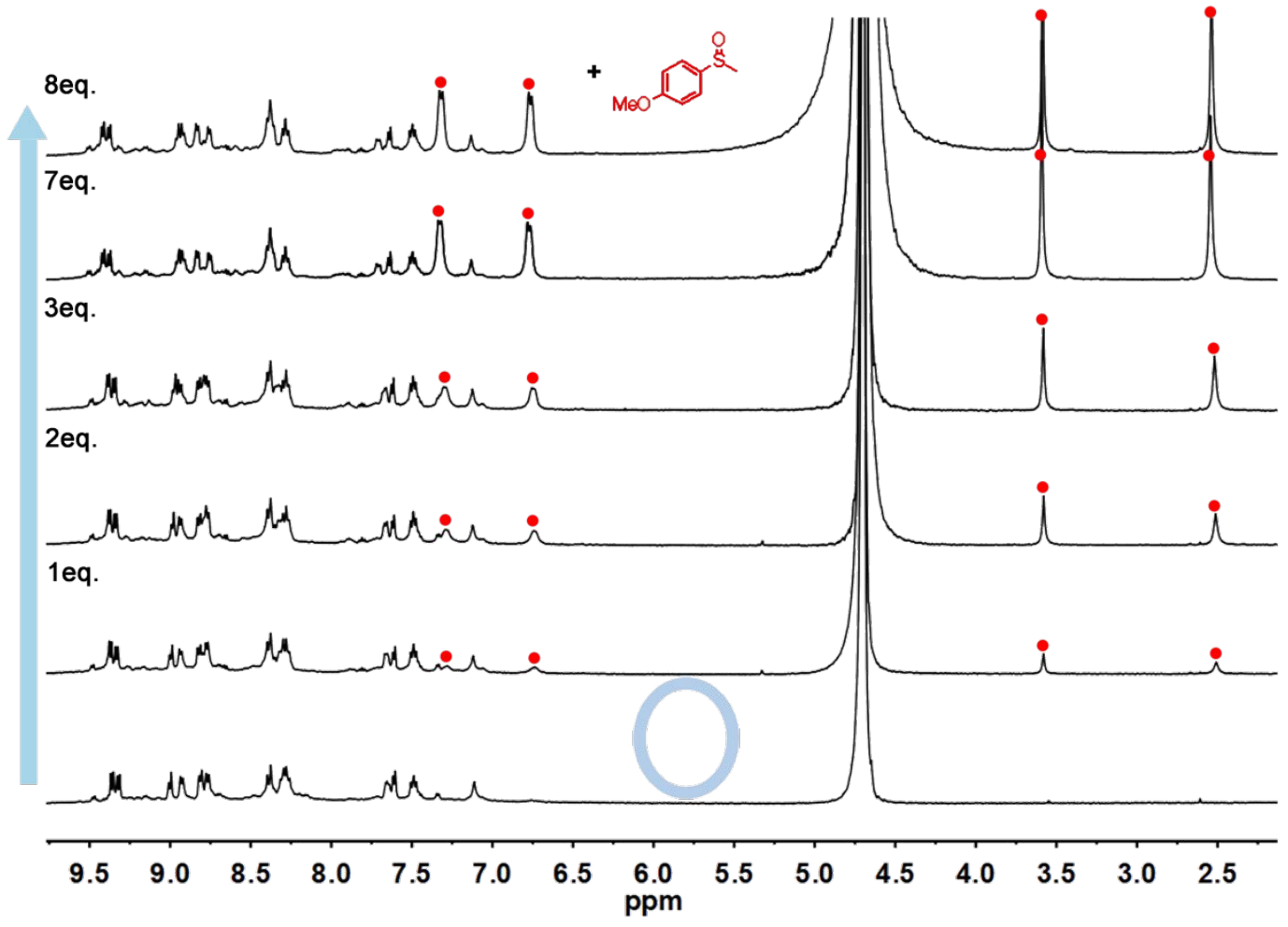

Figure S75. ${ }^{1} \mathrm{H}$ NMR spectra $\left(400 \mathrm{~Hz}, \mathrm{D}_{2} \mathrm{O}, 298 \mathrm{~K}\right)$ of capsule 2 when titrated with product OMSO, which shows only slightly change on host signal. $(\bullet=$ OMSO $)$ 


\section{Crystal data and structure refinement:}

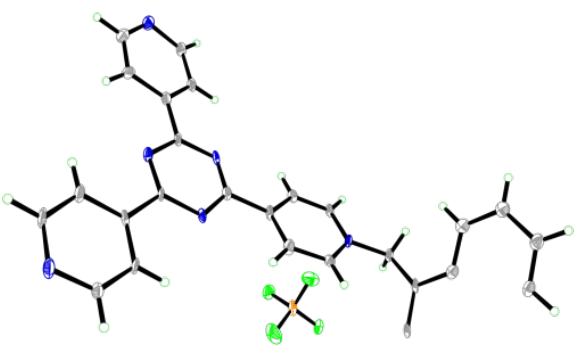

Figure S76. Ortep drawing of the asymmetric unit in the crystal structure of ligand $\mathrm{L}^{2} \cdot\left(\mathrm{BF}_{4}\right)_{2}$ at $30 \%$ probability level.

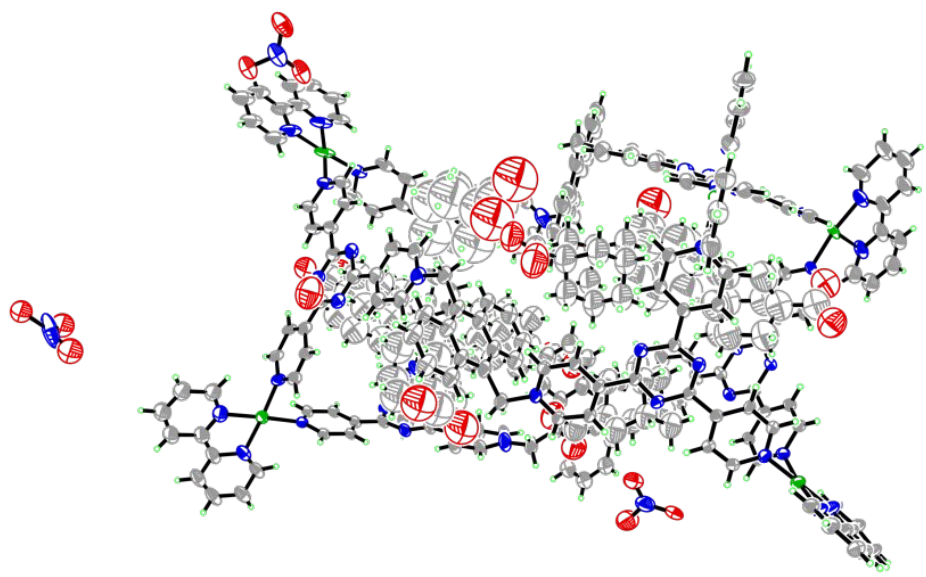

Figure S77. Ortep drawing of the asymmetric unit in the crystal structure (1-adamantanecarboxylic acid) $)_{8} \subset$ capsule 2 of at $30 \%$ probability level.

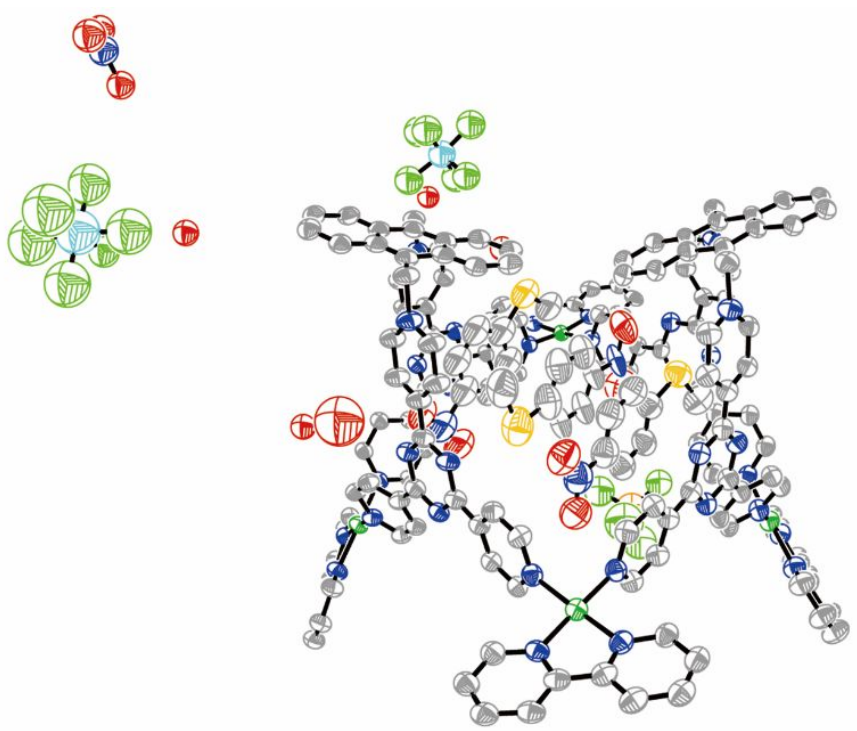

Figure S78. Ortep drawing of the asymmetric unit in the crystal structure (methyl(4-nitrophenyl)sulfane) ${ }_{3} \subset$ bowl 3 of at $30 \%$ probability level. (According to the reported paper ${ }^{56}$ the formation of $\left[\mathrm{SiF}_{6}\right]^{2-}$ is a consequence of hydrolysis of the $\left[\mathrm{BF}_{4}\right]^{-}$anion in water and reaction of fluoride with the glass of the beaker.) 
Supplementary Table 1. Crystal data and structure refinement for ligand $\mathrm{L}^{2}$.

Identification code

Empirical formula

Formula weight

Temperature

Wavelength

Crystal system

Space group

Unit cell dimensions

Volume

Z

Density (calculated)

Absorption coefficient

$\mathrm{F}(000)$

Crystal size

Theta range for data collection

Index ranges

Reflections collected

Independent reflections

Completeness to theta $=22.783^{\circ}$

Refinement method

Data / restraints / parameters

Goodness-of-fit on $F^{2}$

Final $\mathrm{R}$ indices [I $>2 \operatorname{sigma}(\mathrm{I})]$

$\mathrm{R}$ indices (all data)

Extinction coefficient

Largest diff. peak and hole
$\mathrm{L}^{2}$

C52 H36 B2 F8 N12

1002.55

100(2) K

$0.77488 \AA$

Triclinic

P-1

$\mathrm{a}=7.917(3) \AA$

$\alpha=77.05^{\circ}$

$\mathrm{b}=10.411(5) \AA$

$\square \beta=74.62^{\circ}$

$\mathrm{c}=14.793(7) \AA$

$\square \gamma=72.70^{\circ}$

1108.3(9) $\AA^{3}$

1

$1.502 \mathrm{Mg} / \mathrm{m}^{3}$

$0.140 \mathrm{~mm}^{-1}$

514

$0.12 \times 0.05 \times 0.04 \mathrm{~mm}^{3}$

1.576 to $22.783^{\circ}$

$-7<=\mathrm{h}<=7,-9<=\mathrm{k}<=10,-14<=\mathrm{l}<=14$

3970

$2094[\mathrm{R}(\mathrm{int})=0.0482]$

$90.6 \%$

Full-matrix least-squares on $F^{2}$

2094 / 325 / 329

1.133

$R_{1}=0.1236, w R_{2}=0.3842$

$R_{1}=0.1472, w R_{2}=0.3962$

$0.010(6)$

0.514 and -0.506 e. $\AA^{-3}$ 
Supplementary Table 2. Crystal data and structure refinement for (1-adamantanecarboxylic acid) 8

ᄃcapsule 2.

Identification code

Empirical formula

Formula weight

Temperature

Wavelength

Crystal system

Space group

Unit cell dimensions

Volume

Z

Density (calculated)

Absorption coefficient

$\mathrm{F}(000)$

Crystal size

Theta range for data collection

Index ranges

Reflections collected

Independent reflections

Completeness to theta $=20.650^{\circ}$

Refinement method

Data / restraints / parameters

Goodness-of-fit on $F^{2}$

Final $\mathrm{R}$ indices [I $>2 \operatorname{sigma}(\mathrm{I})]$

$\mathrm{R}$ indices (all data)

Extinction coefficient

Largest diff. peak and hole
$\mathbf{A D C}_{8} \subset$ capsule $\mathbf{2}$.

C304 H277 Br N51 O25 Pd6

5763.06

$100 \mathrm{~K}$

$0.77488 \AA$

Monoclinic

$P 2{ }_{1} / c$

$\mathrm{a}=22.611(10) \AA$

$\alpha=90^{\circ}$.

$\mathrm{b}=30.064(14) \AA$

$\beta=94.86^{\circ}$.

$\mathrm{c}=55.373(3) \AA$

$\gamma=90^{\circ}$.

$37506(3) \AA^{3}$

4

$1.021 \mathrm{Mg} / \mathrm{m}^{3}$

$0.524 \mathrm{~mm}^{-1}$

11876

$0.12 \times 0.10 \times 0.06 \mathrm{~mm}^{3}$

0.805 to $20.650^{\circ}$.

$-20<=\mathrm{h}<=20,-27<=\mathrm{k}<=27,-48<=\mathrm{l}<=50$

107402

$29497[\mathrm{R}($ int $)=0.0784]$

$99.5 \%$

Full-matrix least-squares on $F^{2}$

29497 / 5187 / 3144

1.606

$R_{l}=0.1284, w R_{2}=0.3610$

$R_{I}=0.1454, w R_{2}=0.3797$

$\mathrm{n} / \mathrm{a}$

2.448 and -1.617 e. $\AA^{-3}$ 
Supplementary Table 3. Crystal data and structure refinement for (methyl(4-nitrophenyl)sulfane) $)_{3} \subset$ capsule 2 .

Identification code

Empirical formula

Formula weight

Temperature

Wavelength

Crystal system

Space group

Unit cell dimensions

Volume

Z

Density (calculated)

Absorption coefficient

$\mathrm{F}(000)$

Crystal size

Theta range for data collection

Index ranges

Reflections collected

Independent reflections

Completeness to theta $=20.004^{\circ}$

Refinement method

Data / restraints / parameters

Goodness-of-fit on $F^{2}$

Final $\mathrm{R}$ indices [I $>2 \operatorname{sigma}(\mathrm{I})]$

$\mathrm{R}$ indices (all data)

Extinction coefficient

Largest diff. peak and hole
$\mathbf{M N S}_{3} \subset$ bowl 3.

C165 H125 B F16 N36 O14 Pd4 S3 Si2

3728.77

$100(2) \mathrm{K}$

$0.64996 \AA$

Triclinic

$P-1$

$\mathrm{a}=20.535(4) \AA$

$\alpha=70.20^{\circ}$.

$\mathrm{b}=21.230(4) \AA$

$\beta=70.18^{\circ}$.

$c=29.674(6) \AA$

$\gamma=79.95^{\circ}$.

$11425(5) \AA^{3}$

2

$1.084 \mathrm{Mg} / \mathrm{m}^{3}$

$0.414 \mathrm{~mm}^{-1}$

3776

$0.10 \times 0.06 \times 0.04 \mathrm{~mm}^{3}$

0.765 to $20.004^{\circ}$.

$-21<=\mathrm{h}<=21,-22<=\mathrm{k}<=22,-31<=1<=31$

48890

$26300[\mathrm{R}(\mathrm{int})=0.0756]$

$94.2 \%$

Full-matrix least-squares on $F^{2}$

$26300 / 2237$ / 1659

1.474

$R_{I}=0.1519, w R_{2}=0.3886$

$R_{1}=0.1909, w R_{2}=0.4271$

$\mathrm{n} / \mathrm{a}$

1.760 and -0.886 e. $\AA^{-3}$ 


\section{Supplementary reference:}

S1. H.L., Anderson; S., Anderson; J.K.M., Sanders. J. Chem. Soc. Perkin.Trans., 1995, 2231-2245.

S2. S.V., Aathimanikandan; B. S., Sandanaraj; C. G., Arges; C. J., Bardeen and S., Thayumanavan. Org. Let., 2005, 7, 2809-2812.

S3. G. M., Sheldrick. Acta Crystallogr. Sect. A., 2008, 64, 112-122.

S4. A. L., Spek. J. Appl. Crystallogr., 2003, 36, 7-13.

S5. L.X., Cai; S.C., Li; D.N., Yan; L.P., Zhou; F., Guo; Q.F., Sun. J. Am. Chem. Soc., 2018, 140, 4869-4876.

S6. A., Galstyan; P. J. S., Miguel; K. Weisea and B. Lippert. Dalton Trans., 2013, 42, 16151-16161. 